\title{
Thermodynamic Geometric Stability of Quarkonia states
}

\author{
Stefano Bellucci ${ }^{a}$, Vinod Chandra ${ }^{\dagger}$ and Bhupendra Nath Tiwari ${ }^{a \ddagger}$ \\ ${ }^{a}$ INFN-Laboratori Nazionali di Frascati \\ Via E. Fermi 40, 00044 Frascati, Italy. \\ ${ }^{b}$ Department of Theoretical Physics, \\ Tata Institute of Fundamental Research, \\ Homi Bhabha Road Mumbai-400005, India.
}

\begin{abstract}
We compute exact thermodynamic geometric properties of the non-abelian quarkonium bound states from the consideration of one-loop strong coupling. From the general statistical principle, the intrinsic geometric nature of strongly coupled QCD is analyzed for the Columbic, rising and Regge rotating regimes. Without any approximation, we have obtained the non-linear mass effect for the Bloch-Nordsieck rotating strongly coupled quarkonia. For a range of physical parameters, we show in each cases that there exists a well-defined, non-degenerate, curved, intrinsic Riemannian manifold. As the gluons become softer and softer, we find in the limit of the Bloch-Nordsieck resummation that the strong coupling obtained from the Sudhakov form factor possesses exact local and global thermodynamic properties of the underlying mesons, kaons and $D_{s}$ particles.
\end{abstract}

Keywords: Thermodynamic Geometry, Quarkonia, Massive Quarks, QCD Form Factor.

PACS: 02.40.-k; 14.40.Pq; 12.40.Nn; 14.70.Dj

*bellucci@lnf.infn.it

†joshi.vinod@gmail.com

‡bntiwari.iitk@gmail.com 


\section{Introduction}

Quarkonia are the central objects to explore the non-perturbative nature of non-abelian gauge theories 1 . The fate of the heavy quarkonia in hot QCD medium describes the nature of confined-deconfinement phase transition 2 24 in QCD and thereby the nature of the inter-quark forces [5]. An understanding of such issues is essential towards the formation of hot and dense states of quark gluon plasma matter in the heavy ion collision [8] and in the early universe [9]. Thus, our prime focus is to address the stability issues of vacuum constituted by various quarkonia states. This is in direct concern with the fact that the quark matter formation is an important state of art in the particle physics and its behavior is an intense topic of experimental research in the hot QCD media [10. This motivates us to investigate the nature of stability when the quarkonia possess a non-zero mass and spin. Following the notions of the quarkonium vacuum, we notice that an extensive study is required for a proper understanding of the thermodynamic stability and global statistical correlation in the non-abelian gauge theories. To the best of our knowledge, this is perhaps the first attempt in this direction.

It is by now well established that the thermodynamic intrinsic geometric examinations are important in the configurations involving black holes in string theory [11 17] and $M$-theory [18 21, possessing a set of rich stability structures $22 \sqrt{26}$. There exists a wealth of literature where thermodynamic geometry plays an important role towards the understanding the physics of black-holes, black branes, black rings and various higher dimensional objects. Attentions were paid on the equilibrium perspective of black holes, and thereby one explicates the nature of the pair correlations and associated stability of the solutions. Recently, the thermodynamic geometry has emerged as one of the most powerful tool in the understanding covariant statistical correlations and the limit of the underlying microscopic-macroscopic duality issues for the $1 / 2$ - BPS black holes in string theory [26. There have been several general notions explored in the condensed matter physics 27 34, motivating us to consider the quarkonia with a set of equilibrium parameters and analyze the underlying parametric pair correlation functions and global correlations. We find thus that the intrinsic geometric investigation possesses an exact mathematical nature of the massive fluctuating quarkonia. In this concern, we provide a brief account of the concerned strong interactions and thermodynamic geometry in the next section.

Given a definite covariant intrinsic geometric description of the quarkonia, we examine (i) the conditions of thermodynamic stability of the configuration, (ii) its parametric global correlation function in terms of a chosen set of fluctuating parameters. In this analysis, one can enlist the complete set of non-trivial parametric correlation functions of the quarkonia. The similar considerations remain valid over the black hole configurations in general relativity [35] 38, attractor black holes [39 43] and Legendre transformed chemical configurations [44, 45]. Interestingly, we have explored the issue of thermodynamic geometry to the hot QCD [46] and offered the detailed picture of such duality relations between the thermodynamic geometry and quark-number susceptibilities. The issue of the quark matter stability is quite novel in its own right and calls for further investigations of the thermodynamic geometric structure of the phases of non-abelian gauge theories and quark matter production 47 .

The thermodynamic geometric consideration is examined for the strong coupling of massless quarks which reveal limited effects of the QCD calculations, which have been known for the gluons since decades 48,49. The intrinsic geometric analysis of the linear Regge rotating quarkonium configuration [50] offers the thermodynamic stability of quark matter with a precise account of non-zero Regge mass. This consideration supports the formation of the topological Heisenberg spin glass [51] and vector boson pair production at the hadron colliders [52]. Further, the thermodynamic geometric properties take an intrinsic account of the proton-proton and proton-antiproton scattering, hadronic total cross sections through the soft gluon summation in impact parameter space [53, and production of the gluons [54]. Thus, the differential geometry plays an important role in the study of the physics of quarkonia.

In this paper, we examine the thermodynamic stability properties of the quarkonia system described by two and three parameters. We find that our analysis uniformly applies to all possible QCD particles with varying masses from the range of the charmonium states to the bottomonium states. This follows from the fact that the effective mass of the theory is kept very arbitrary; therefore the finite temperature behavior of the quarkonia is captured through the present analysis. From the thermodynamic geometric viewpoint of the hot QCD [46, it seems quite interesting that we can reveal the near equilibrium behavior of quarkonia over an ensemble of hot QCD states. To do so, we introduce the thermodynamic metric tensor through the variations of the strong coupling, and focus our attention on the thermodynamic properties when gluons become softer and softer [53]. Our consideration reveals the thermodynamic geometric nature of very soft gluons and thereby describes the stability properties of non-abelian quarkonium bound states with and without the mass.

As explained in the section 2, the problem thus posed starts from the very definition of Sudhakov form 
factor [55], and thus the intrinsic geometric understanding of the QCD coupling in the limit of the vanishing transverse momentum energies. Notice that our analysis considers all types of QCD states, as we do not truncate the theory but work with the full Bessel function underlying in the Sudhakov form factor. We initiate our analysis where the computation requires only the first few excited states in which one may think that only the charmonium states play the dominant role in the quark matter formation. The analysis is continued for the mass-less Regge rotating quarkonia, and finally all possible mass states are considered through the strong coupling. Our study is two folded, (i) the investigations on the zero temperature properties of quarkonia, and (ii) extensions to the finite temperature, as well as very high temperature properties of the quarkonium vacuum fluctuations in the non-abelian gauge theories. This shows the universality of our consideration of the thermodynamic geometry, that applies for all possible mass excitations of the quarkonium vacuum, and thus the stability of quark matter formations.

The effect of finite mass is explored for the Regge mass and arbitrary mass quarkonia with the one-loop Bloch-Nordsieck resummation [56]. Following the Polyakov arguments [50, our analysis shows that the rising potential vacuum can be thought of as a collection of decaying and weakly interacting particles. However, the Regge rotating configuration offers almost the same stability structure and is also nearly weakly interacting. Subsequently, we show that the global thermodynamic correlation of the Regge rotating states remains almost the same as that of the massless states, up to the sign of the rotation term. With the large quark mass, the strong coupling acquires a large twist and thus thermodynamically the configuration becomes highly unstable. For experimental reasons, the graphical characterizations are offered for large QCD cut-off, where the quarkonium vacuum attains the Regge rotating regime. Such an investigation clarifies the thermodynamic stability properties of the quark matter formation. With this motivation, we shall focus our attention on the (i) quark masses and their dependence on the decay constants, (ii) soft gluons and meson decays, (iii) very soft gluons and (iv) strong coupling hadronic form factor.

The paper is organized as follows. In section 2, we present a brief account of the strong interactions present in the quarkonium formation and its thermodynamic geometry. In section 3, we consider the non-rotating massless quarks and examine their thermodynamic geometric stabilities. Subsequently, we extend the analysis for the non-zero angular momentum. In section 4, we explore generic cases in the framework of the Sudhakov form factor in the limit of Bloch-Nordsieck resummation for the massive quarkonia, with and without rotations. In section 5, we discuss some concluding issues towards the thermodynamic geometric picture of quark matter formation and the stability of underlying QCD particles.

\section{Phases of the Strong Interaction}

In this section, we shall set-up the formulation of problem and outline the notion of the intrinsic thermodynamic geometry. The non-perturbative gluonic effects gives the relation of effective QCD theories with the index of the potential, momentum scale, mass and angular momentum, if any, and thus the consideration for the analysis. Most efficiently, our intrinsic geometric model is designed to provide the critical values for the parameters of the gluonic effects. It is worth mentioning the importance of the present method that the remaining values of the parameters would be an stable mode for the considered type of quarkonia.

\subsection{Quarkonium Bound States}

In the physics of quarks, the non-perturbative gluonic effects are addressed via the considerations of Bloch and Nordsieck [56. Some quantities of known interest are intrinsic transverse momentum of Drell-Yan pairs [57, impact parameter distribution of partons and rise of total cross section [58, form factors and decay constants [59] of the particles. A gluon is said to be soft, if the transverse momentum $k_{\perp}$ is kept small. In this limit, the infrared limit of the charmonium is always unobservable. This follows from the fact that a momentum integration is required to perform, if one wants to keep a vanishingly small transverse momentum, i.e., $k_{\perp} \rightarrow 0$ for the gluons. In the case of abelian theory, the results follow directly from the properties of the underlying Poisson distribution and their numerical counterparts.

In the case of non-abelian theory, the consideration of Sudhakov form factor turns out to be an efficient tool for the soft gluons, which is described as below. Let us recall that the QCD coupling $\alpha_{s}\left(k_{\perp}\right)$ never lies near the limit $k_{\perp} \rightarrow 0$, and thus the QCD effects are limited for the bound state thus formed after the resummation. This initiates the requirement for the integration over the $k_{\perp}$ and thus the necessity of Sudhakov form factor. Let us illustrate the computations for decays processes associated with the pions, viz., $\pi^{0} \rightarrow \gamma \gamma$ and $\pi \rightarrow \mu \nu$. 
During the process, when a virtual photon hits the quarks, soft gluons are emitted with $k_{\perp} \rightarrow 0$ and a non-zero total momentum $k^{2} \neq 0$, satisfying the standard transverse momentum Poisson distribution $d^{2} P\left(k_{\perp}\right)$. The associated Poisson summed vertex and the transverse limiting momenta are respectively given by

$$
\begin{aligned}
\Gamma_{\pi \rightarrow \gamma \gamma} & \sim \frac{d^{2} P\left(k_{\perp}\right)}{d k_{\perp}^{2}} \\
\Pi(0) & =\left.\frac{d^{2} P\left(k_{\perp}\right)}{d k_{\perp}^{2}}\right|_{k_{\perp}=0} \\
& =\int d^{2} \vec{b} \exp (-h(b))
\end{aligned}
$$

where the exponent defined as

$$
h(b)=\int d^{3} n_{k}\left(1-\exp \left(-i \vec{b} \cdot \overrightarrow{k_{\perp}}\right)\right)
$$

is known the Sudhakov form factor. Recall that one is required to take the vanishing transverse momentum limit for a decay, which is done systematically with the help of the Sudhakov form factor, see for a review 60. We now illustrate the case of massless quarks. For the quark mass $m_{q}=0$, in turns out that the Sudhakov form factor reduces to the following integral

$$
h(b)=\int \frac{d k_{l}}{2 k} \frac{d k_{\perp}}{k_{\perp}^{2}}\left(1-\exp \left(-i \overrightarrow{k_{\perp}} \cdot \vec{b}\right)\right),
$$

where $k_{\perp} \in\left(0, m_{P / 2}\right)$ is due to a physical reason that on average each soft gluon can take as much as half of the initial center of mass energy. Herewith, the consideration of Sudhakov form factor comes for a model of limiting transverse momentum QCD coupling $\left.\alpha_{s}\left(k_{\perp}\right)\right|_{k_{\perp} \rightarrow 0}$. Inspired by the behavior of relativistic wave functions near the origin for a QCD potential 61,62 for the Rechardson potential for the quark bound states, one arrives at the transverse momentum dependent strong QCD coupling

$$
\alpha_{s}\left(k_{\perp}^{2}\right)=\frac{12 \pi}{\left(33-2 N_{f}\right)} \frac{p}{\ln \left(1+p\left(\frac{k_{\perp}}{\Lambda_{Q C D}}\right)^{2 p}\right)}
$$

in the limit of one loop gluon exchange potential. Towards the determination of the index $p$, an interesting argument follows from the consideration of Polyakov [50] which we shall explore further from the perspective of thermodynamic geometry in the subsequent discussion of the present paper. Before doing so, let us consider the joint effects of the (i) confinement and (ii) rotation, and make a platform to describe the properties of the thermodynamic geometry. In this case, the configuration is described by Regge trajectories with the leading order effective potential

$$
V(r, J)=\frac{J(J+1)}{r^{2}}+C r^{2 p-1} .
$$

Such an effective potential is inspired from the limiting QCD strong coupling

$$
\alpha_{s}\left(Q^{2}\right)=b^{-1} \frac{p}{\ln \left(1+p\left(\frac{Q^{2}}{\Lambda_{Q C D}^{2}}\right)^{p}\right)}
$$

with the matching of the pre-factor as $b:=\left(33-2 N_{f}\right) / 12 \pi$. In the momentum space, the net effective potential offers the right quarkonium bound states, after taking account of the one-loop exchange terms. In the sense of one-loop quantum effects, we propose that the "quantum" nature of quark matters follows directly from the consideration of the thermodynamic intrinsic geometric potential of Richardson types. The index $p$ defines the nature of the potential [50, i.e. whether the quarkonium lies in the Coulomb phase $(p=0)$ or in the rising phase $(p>1 / 2)$. Notice further that the Regge behavior may also be determined from interpolating values of the index $p$, which is the matter of the subsequent sections. For the future purpose, it is worth mentioning that the minimization of the effective potential containing the confining and rotation effects yields the value $p=5 / 6$ for the index, which corresponds to the linear Regge regime. We shall exploit these facts, while dealing with the thermodynamic geometry of rotating (massive) quarkonia. 


\subsection{Thermodynamic Geometry}

In this subsection, we offer a brief account of the essential features of thermodynamic geometries from the perspective of the application to the strongly coupled QCD. We shall focus our attention on the thermodynamic geometric nature of quarkonium bound states with a finite number of parameters carried by the effective field theories.

Let us consider the framework of the intrinsic Riemannian geometry whose covariant metric tensor is defined as the Hessian matrix of the QCD coupling, with respect to a finite number of arbitrary parameters carried by the soft gluons and quarks. This consideration yields the space spanned by the $n$ parameters of the strong QCD coupling $\alpha_{s}$, which in the present consideration, exhibits a n-dimensional intrinsic Riemannian manifold $M_{n}$. Following the notion of 24 32] for the thermodynamic geometry, the components of the covariant metric tensor are defined as

$$
g_{i j}:=\frac{\partial^{2} \alpha_{s}(\vec{x})}{\partial x^{j} \partial x^{i}},
$$

where the vector $\vec{x} \in M_{n}$. In the quarkonium effective configuration, there are only a few in physical parameters which make the analysis fairly simple. As mentioned in the foregoing section, the variables of the interest in the present study of quarkonium are the momentum scale parameter, $Q^{2}:=q$, the mass $M$ and the angular momentum $J$, if any. Thus, let us first illustrate the consideration of thermodynamic geometry for the non-rotating configurations, viz., $J=0$. In this case, we can take the QCD coupling $\alpha_{s}(q, p)$ as a function of $q, p$ and thereby may explore the local and global thermodynamic properties towards the stability of the quarkonia on $q p$-surface. For a given QCD coupling $\alpha_{s}(q, p):=A$, an equilibrium quarkonium configuration is achieved at the points, where the first derivatives

$$
\frac{\partial A(q, p)}{\partial q}=0, \frac{\partial A(q, p)}{\partial p}=0
$$

vanish identically, implying the existence of the equilibrium data $\left\{q_{0}, p_{0}\right\}$ on an intrinsic Riemannian surface $\left(M_{2}(R), g\right)$. In order to verify whether the quarkonium configuration is minimally coupled at $\left\{q_{0}, p_{0}\right\}$, we may follow the set-up of thermodynamic geometry and define the components of the thermodynamic metric tensor as

$$
g_{q q}=\frac{\partial^{2} A}{\partial q^{2}}, \quad g_{q p}=\frac{\partial^{2} A}{\partial q \partial p}, \quad g_{p p}=\frac{\partial^{2} A}{\partial p^{2}}
$$

In the present case of the $\left(M_{2}(R), g\right)$, it follows that the determinant of the metric tensor is

$$
\|g(q, p)\|=A_{q q} A_{p p}-A_{q p}^{2} .
$$

Explicitly, we can calculate $\Gamma_{i j k}, R_{i j k l}, R_{i j}$ and $R$ for the above two dimensional thermodynamic geometry $\left(M_{2}, g\right)$ of the non-rotating quarkonia and may easily see that the scalar curvature is given by

$$
\begin{aligned}
R(q, p)= & -\frac{1}{2\|g(q, p)\|^{2}}\left(A_{p p} A_{q q q} A_{q p p}-A_{q q} A_{q p p}^{2}-A_{p p} A_{q q p}^{2}\right. \\
& \left.+A_{q p} S_{q q p} A_{q p p}+A_{q q} A_{q q p} A_{p p p}+A_{q p} A_{q q q} A_{p p p}\right) .
\end{aligned}
$$

As a global intrinsic geometric invariant, the scalar curvature accompanies information of the correlation volume of underlying quark fluctuations. The scalar curvature further explicates the nature of long range global correlations and phase transitions, if any, deriving from a given phase. In this sense, we anticipate that the set of particles corresponding to the specific decay, are statistically interacting, if the underlying quarkonium configuration has a non-zero thermodynamic scalar curvature. Incrementally, we notice that the configurations under present consideration are allowed to be effectively attractive or repulsive, and weakly interacting in general. For the two dimensional thermodynamic geometry [24] defined as the intrinsic Riemannian surface 
$\left(M_{2}(R), g\right)$, the relation of the thermodynamic scalar curvature to the thermodynamic curvature tensor is given as

$$
R(q, p)=\frac{2}{\|g\|} R_{q p q p} .
$$

The intrinsic geometric analysis thus provides a set of physical indications encoded in the geometric quantities, e.g., the scalar curvature and possible geometrically non-trivial invariants. For a given quarkonium configuration, the underlying analysis would involve an ensemble or subensemble equilibrium configuration forming the statistical basis for the Gaussian distribution of the particle, which is ensured for any distribution in the late time limit. With this brief introduction to the thermodynamic geometry, we shall now proceed to systematically analyze the underlying stability structures for the parametric fluctuations of the massless non-rotating quarkonia.

\section{Massless Quarkonia}

In the present section, we shall examine the nature of the QCD coupling $\alpha_{s}(q, p)$ from the thermodynamic stability properties. Firstly, we analyze the massless quarkonium for non-rotating configurations and then take take an account of the rotation in the next subsection. In the subsequent analysis, we shall adopt the notation $L=\Lambda_{Q C D}^{2}$

\subsection{Non-rotating Quarkonia}

As stated earlier, the thermodynamic metric in the quarkonium parameter space is given by the Hessian matrix of the strong coupling with respect to the intensive variables, which in this case are the two distinct parameters $\{q, p\}$ carried by the quark matters. Considering Eqn.(7), one obtains the following expression:

$$
A(q, p):=\frac{p}{b \ln \left(1+p(q / L)^{p}\right)}
$$

for the strong QCD coupling. To compute the thermodynamic metric tensor in the parameter space, we employ the Eqn.(10), which leads to the following expression for the components of the metric tensor

$$
g_{q q}=\frac{p^{3}}{b q^{2}} \frac{n_{11}^{Q}}{r_{11}^{Q}}, \quad g_{q p}=\frac{p^{2}}{b q} \frac{n_{12}^{Q}}{r_{12}^{Q}}, \quad g_{p p}=\frac{1}{b} \frac{n_{22}^{Q}}{r_{22}^{Q}}
$$

In this framework, we observe that the geometric nature of parametric pair correlations offers the notion of fluctuating quarkonia. In order to simplify the subsequent notations, let us define the logarithmic factor as

$$
l(p):=\ln \left(1+p(q / L)^{p}\right) .
$$

In this case, we find that the factors in the numerator of the local pair correlation functions are expressed as follows

$$
\begin{aligned}
n_{11}^{Q}:= & 2 p^{2}(q / L)^{2 p}+l(p)\left((q / L)^{p}-p(q / L)^{p}+(q / L)^{2 p} p\right) \\
n_{12}^{Q}:= & 2(q / L)^{2 p} p+l(p)\left(2(q / L)^{2 p} p^{2} \ln (q / L)-3(q / L)^{p}\right. \\
& \left.-2(q / L)^{2 p} p-(q / L)^{p} p \ln (q / L)\right) \\
n_{22}^{Q}:= & 2(q / L)^{2 p}\left(p+2 p^{2} \ln (q / L)+p^{3} \ln (q / L)^{2}\right) \\
& -l(p)\left((q / L)^{p}+(q / L)^{2 p} p+4(q / L)^{p} p \ln (q / L)\right. \\
& \left.+2(q / L)^{2 p} p^{2} \ln (q / L)+(q / L)^{p} p^{2} \ln (q / L)^{2}\right) .
\end{aligned}
$$

We further notice an interesting conclusion for the denominator of the local pair correlation functions, and we find for the massless non-rotating configuration that the denominators $\left\{r_{i i}^{Q} \mid i=1,2,3\right\}$ of all the local pair correlation functions take uniform value $l(p)^{3} \exp (2 l(p))$.

Thus, the fluctuating quarkonium configuration may be easily analyzed in terms of the parameters of the underlying effective theory. Moreover, it is evident that the principle components of the metric tensor, signifying 


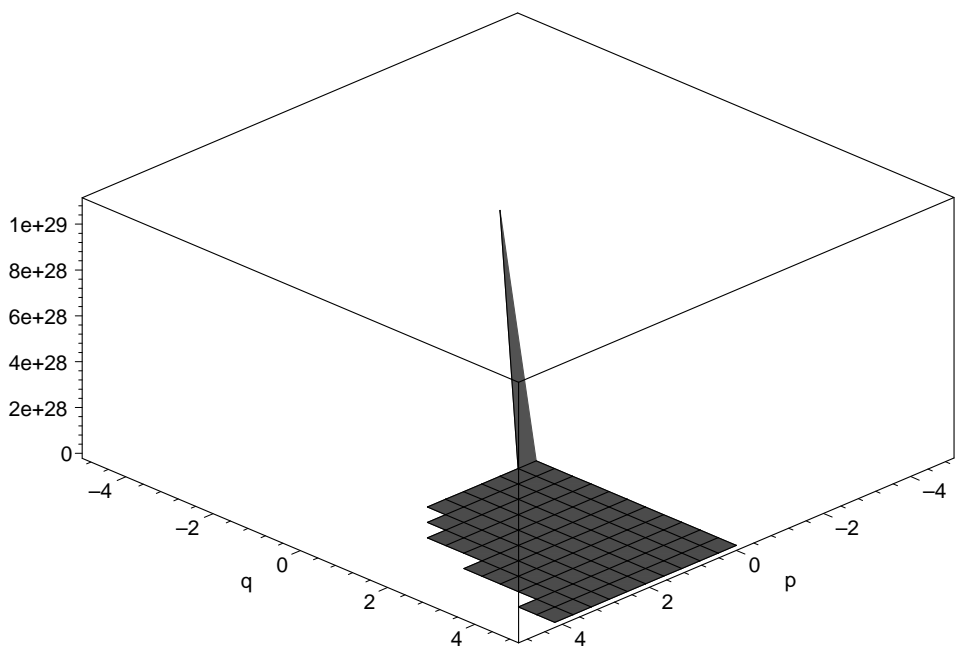

Figure 1: The determinant of the metric tensor plotted as a function of the scale and index parameter $q, p$, describing the fluctuations in massless non-rotating quarkonia.

self pair correlations remain positive definite functions. In a given QCD phase, this happens when the parameters $\{q, p\}$ are confines in the domain

$$
\left\{\mathcal{D}:=(q, p) \in M_{2} \mid n_{11}^{Q}>0, n_{22}^{Q}>0\right\}
$$

Over this domain of $\{q, p\}$, it is worth mentioning that the massless non-rotating quarkonia is well-behaved and locally stable. The global stability is offered by computing the determinant of the metric tensor, and requiring it to be positive definite. Following the Eqn.(11), we find further for the generic value of the parameters that the Gaussian fluctuations form a stable set of correlations over $\{q, p\}$, if the determinant of the metric tensor

$$
\|g\|=\frac{p^{3}}{b^{2} q^{2} l(p)^{5} \exp (3 l(p))} n_{g}^{Q}
$$

remains a positive function on the intrinsic $q p$-surface $\left(M_{2}(R), g\right)$. Explicitly, we obtain that the numerator of the determinant of the metric tensor can be expressed as

$$
\begin{aligned}
n_{g}^{Q}(q, p):= & 2(q / L)^{3 p}\left(p+3 p^{2}+2 p^{2} \ln (q / L)+2 p^{3} \ln (q / L)+p^{3} \ln (q / L)^{2}\right) \\
& -l(p)\left(2(q / L)^{2 p}+p(q / L)^{3 p}+2 p^{2}(q / L)^{3 p} \ln (q / L)+4 p^{2}(q / L)^{3 p}\right. \\
& +4 p(q / L)^{2 p} \ln (q / L)+p^{2}(q / L)^{2 p} \ln (q / L)^{2}+2 p^{2}(q / L)^{2 p} \ln (q / L) \\
& \left.+7 p(q / L)^{2 p}\right) .
\end{aligned}
$$

The behavior of the determinant of the metric tensor shows that such massless non-rotating quarkonium becomes unstable for opposite values of $q$ and $p$ for positive $n_{g}^{Q}(q, p)$. For generic $q$ and $p$, the nature of the determinant of the metric tensor is depicted in the Eqn.(19), showing that the quarkonia become unstable in the vanishing limit of $q$ or $p$. It is worth mentioning for a common sigh of the index parameter $p$ and $b$, that the non-rotating massless quarkonia is stable in the regions of the $\left(M_{2}(R), g\right)$, where the function $n_{g}^{Q}(q, p)$ picks up the positive sign.

Let us explore the functional behavior of the associated scalar curvature, in order to examine the mixing effects of the parameters forming the intrinsic surface. In this case, there is only one non-trivial component of the Riemann Christoffel tensor $R_{q p q p}$. As per the definition of the Eqn.(12), our computation shows that the scalar curvature reduce to the following specific form

$$
R(q, p)=\frac{b l(p)}{2 p^{2}\left(n_{g}^{Q}\right)^{2}}\left(n_{R}^{(0) Q}+n_{R}^{(1) Q} l(p)+n_{R}^{(2) Q} l(p)^{2}+n_{R}^{(3) Q} l(p)^{3}\right) .
$$




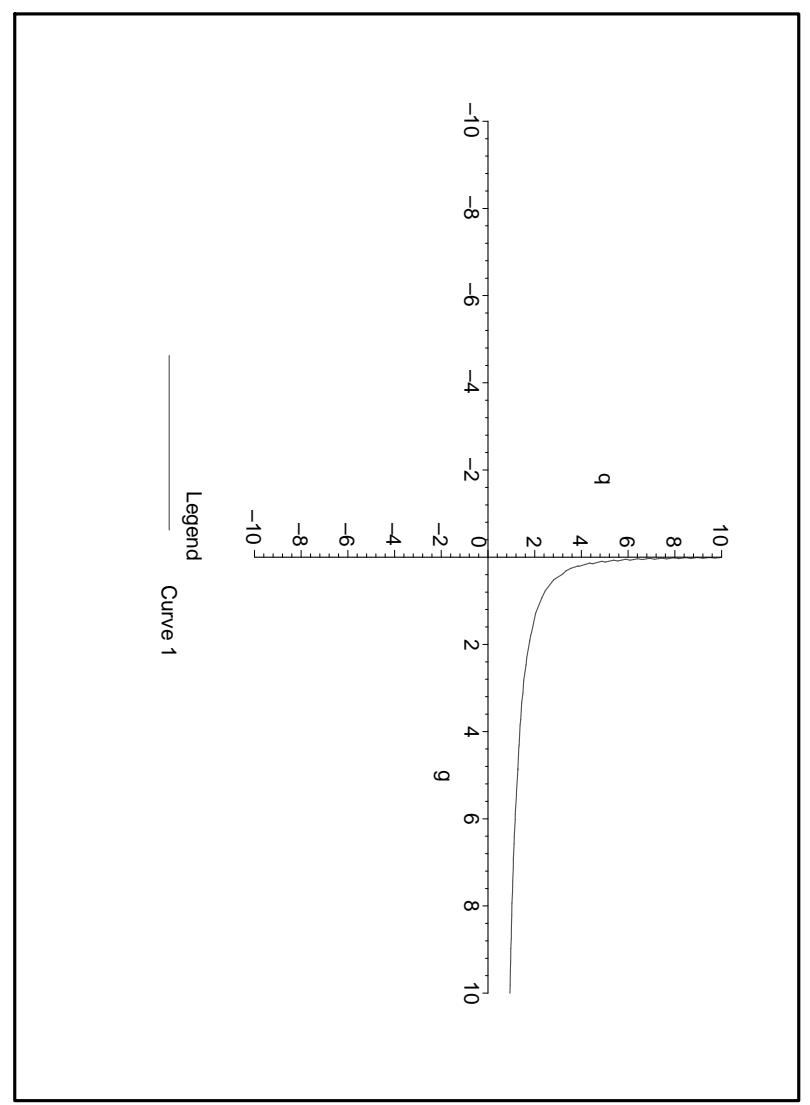

Figure 2: The determinant of the metric tensor plotted as a function of $q$, describing the fluctuations in massless non-rotating quarkonia near the Coulomb regime. 


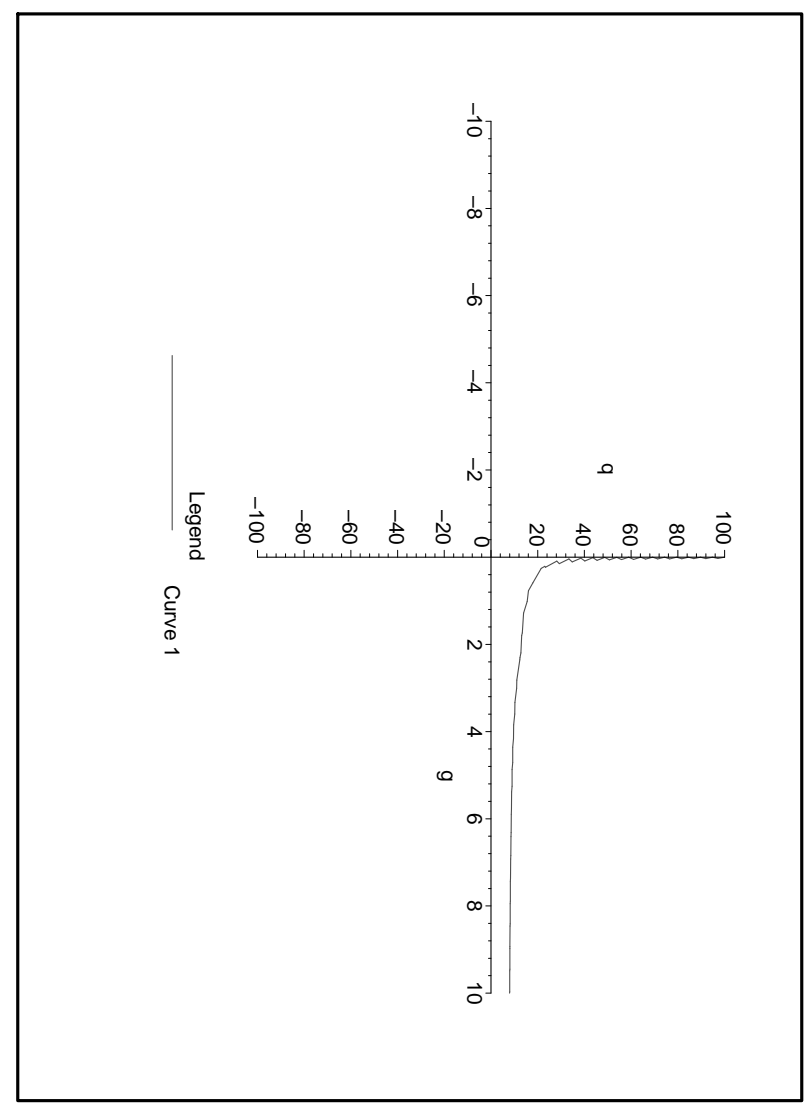

Figure 3: The determinant of the metric tensor plotted as a function of $q$, describing the fluctuations in massless non-rotating quarkonia in the raising regime. 
The factors of the numerator of the scalar curvature take the following expressions

$$
\begin{aligned}
& n_{R}^{(0) Q}=\ln (q / L)^{4}-16(q / L)^{2 p} p^{4} \ln (q / L)-16(q / L)^{2 p} p^{7} \ln (q / L)^{3} \\
& -56(q / L)^{2 p} p^{6} \ln (q / L)^{2}-48(q / L)^{2 p} p^{6} \ln (q / L)-4(q / L)^{2 p} p^{7} \ln (q / L)^{4} \\
& -16(q / L)^{2 p} p^{7} \ln (q / L)^{2}-16(q / L)^{2 p} p^{6} \ln (q / L)^{3}-64(q / L)^{2 p} p^{5} \ln (q / L) \\
& -24(q / L)^{2 p} p^{5} \ln (q / L)^{2}-36(q / L)^{2 p} p^{5}-4(q / L)^{2 p} p^{3}, \\
& n_{R}^{(1) Q}=28 p^{6}(q / L)^{2 p} \ln (q / L)^{2}+14 p^{4}(q / L)^{2 p}+20 p^{5}(q / L)^{2 p} \ln (q / L)^{2} \\
& +50 p^{3}(q / L)^{p}+40 p^{3}(q / L)^{p} \ln (q / L)+146 p^{4} \ln (q / L)(q / L)^{p} \\
& +56 p^{4}(q / L)^{p} \ln (q / L)^{2}+8 p^{6}(q / L)^{2 p} \ln (q / L)^{3}+4 p^{3}(q / L)^{2 p} \\
& +10 p^{2}(q / L)^{p}+48(q / L)^{2 p} p^{5}+102(q / L)^{p} p^{4}+112 p^{5}(q / L)^{p} \ln (q / L)^{2} \\
& +32(q / L)^{2 p} p^{6} \ln (q / L)+24(q / L)^{p} p^{6} \ln (q / L)^{2}+32 p^{5}(q / L)^{p} \ln (q / L)^{3} \\
& +104(q / L)^{p} p^{5} \ln (q / L)+24(q / L)^{p} p^{6} \ln (q / L)^{3}+6(q / L)^{p} p^{6} \ln (q / L)^{4} \\
& +16 p^{4}(q / L)^{2 p} \ln (q / L)+50 p^{5} \ln (q / L)(q / L)^{2 p}, \\
& n_{R}^{(2) Q}=-6 p^{3}(q / L)^{2 p}-8 p^{5}(q / L)^{2 p} \ln (q / L)-6 p^{4} \ln (q / L)(q / L)^{2 p} \\
& +6 p^{4}(q / L)^{2 p}+10 p^{3}(q / L)^{p}-14 p^{2}(q / L)^{p}-20 p^{2}-12 p \\
& -30 p^{2} \ln (q / L)+8(q / L)^{p} p^{6} \ln (q / L)^{2} l(p)^{2}-20 p^{3}(q / L)^{p} \ln (q / L) \\
& -4 p^{5}(q / L)^{2 p} \ln (q / L)^{2} l(p)^{2}-90 p^{3} \ln (q / L)-36 p^{3} \ln (q / L)^{2} \\
& -62(q / L)^{p} p^{4} l(p)^{2}-16(q / L)^{2 p} p^{5}-16 p^{4} \ln (q / L)^{3} \\
& -8 p^{5} \ln (q / L)^{2}-56 p^{4} \ln (q / L)-56 p^{4} \ln (q / L)^{2} \\
& -8 p^{5} \ln (q / L)^{3}-2 p^{5} \ln (q / L)^{4}-8(q / L)^{p} p^{5} \ln (q / L) \\
& +8(q / L)^{p} p^{6} \ln (q / L)^{3}+2(q / L)^{p} p^{6} \ln (q / L)^{4}-82 p^{3} \\
& -16 p^{4}(q / L)^{p} \ln (q / L)^{2}-34 p^{4} \ln (q / L)(q / L)^{p}, \\
& n_{R}^{(3) Q}=-15 p^{3} \ln (q / L)+2 p^{3}(q / L)^{2 p}-4 p^{4}(q / L)^{2 p}-8 p^{5} \ln (q / L)^{2}(q / L)^{p} \\
& -9 p^{4} \ln (q / L)(q / L)^{p}-2 p^{4} \ln (q / L)^{2}(q / L)^{p}-2 p^{5} \ln (q / L)^{3}(q / L)^{p} \\
& +p^{3} \ln (q / L)(q / L)^{p}-8 p^{5} \ln (q / L)(q / L)^{p}-20 p^{3}(q / L)^{p}+9 p^{2}(q / L)^{p} \\
& -5 p^{4} \ln (q / L)^{3}-4 p^{5} \ln (q / L)^{2}-16 p^{4} \ln (q / L)-19 p^{4} \ln (q / L)^{2} \\
& -4 p^{5} \ln (q / L)^{3}-p^{5}+16(q / L)^{-p} p^{2}-6(q / L)^{-p} p+5(q / L)^{-p} p^{2} \ln (q / L)^{2} \\
& +(q / L)^{-p} p^{3} \ln (q / L)^{3}+10(q / L)^{-p} p^{2} \ln (q / L)+3(q / L)^{-p} p^{3} \ln (q / L)^{2} \\
& +6(q / L)^{-p} p \ln (q / L)+4(q / L)^{-p} p^{3} \ln (q / L)+6(q / L)^{-p}-28 p^{2} \\
& -24(q / L)^{2 p} p^{4}+7 p^{3}-3 p^{3} \ln (q / L)^{2}+3 p^{2} \ln (q / L)+12 p .
\end{aligned}
$$

In the case when the Ricci scalar curvature $R(q, p)$ vanishes, the underlying quarkonium system is found to be in equilibrium. Such a state of the configuration can arise with $\left\{n^{(i) Q}=0, i=0,1,2,3\right\}$, if the other factors of the scalar curvature remain non-zero. In the other case, when the $R(q, p)$ diverges, the configuration goes over a transition. Such an extreme behavior of the quarkonia is expected to happen, when either the index $p$ or the the numerator of the determinant of the metric tensor $n_{g}^{Q}$ vanish. Geometrically, the intrinsic $q p$-surface becomes the flat Euclidean plane in the first case, while it gets infinitely curved in the second one.

For the choice of QCD parameters $L=1000$ and $b=1$, the Fig.(11) shows the plot of the determinant of the metric tensor. This plots explicates the nature of the stability of non-rotating configurations in the limit of massless quarkonia. The corresponding plot for the scalar curvature is depicted in the Fig.(4). This plot shows the global nature of the non-rotating configuration in the limit of massless quarkonia, under the effects of Gaussian fluctuations of the index $p$ and the parameter $q$. Notice, for the generic massless nonrotating quarkonium configurations, that the limiting scalar curvature interestingly simplifies to the slice shape of Fig.(11). Further, it is worth mentioning that the corresponding peaks of the determinant of the metric tensor, as depicted in the Fig.(1) indicate the graphical nature of the instability present in the general non-rotating massless quarkonia. Physically, the presence of peaks in the determinant of the metric tensor shows a non-trivial interaction in the system.

For the regime of the Coulambic potential, the respective surface plots of the determinant of the metric tensor and scalar curvature are respectively shown in the Figs.(2) and (5) for $p=0.1$. We observe that the stability 


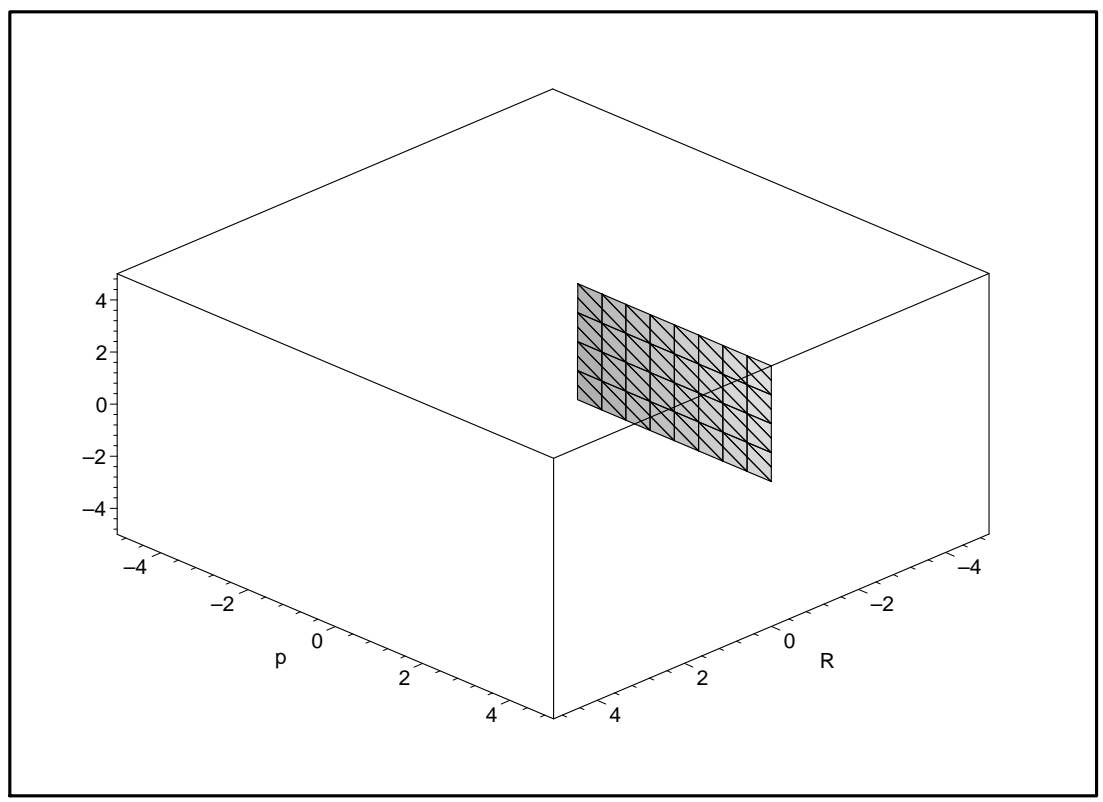

Figure 4: The curvature scalar plotted as a function of $q$, and the index parameter $p$, describing the fluctuations in massless non-rotating quarkonia.

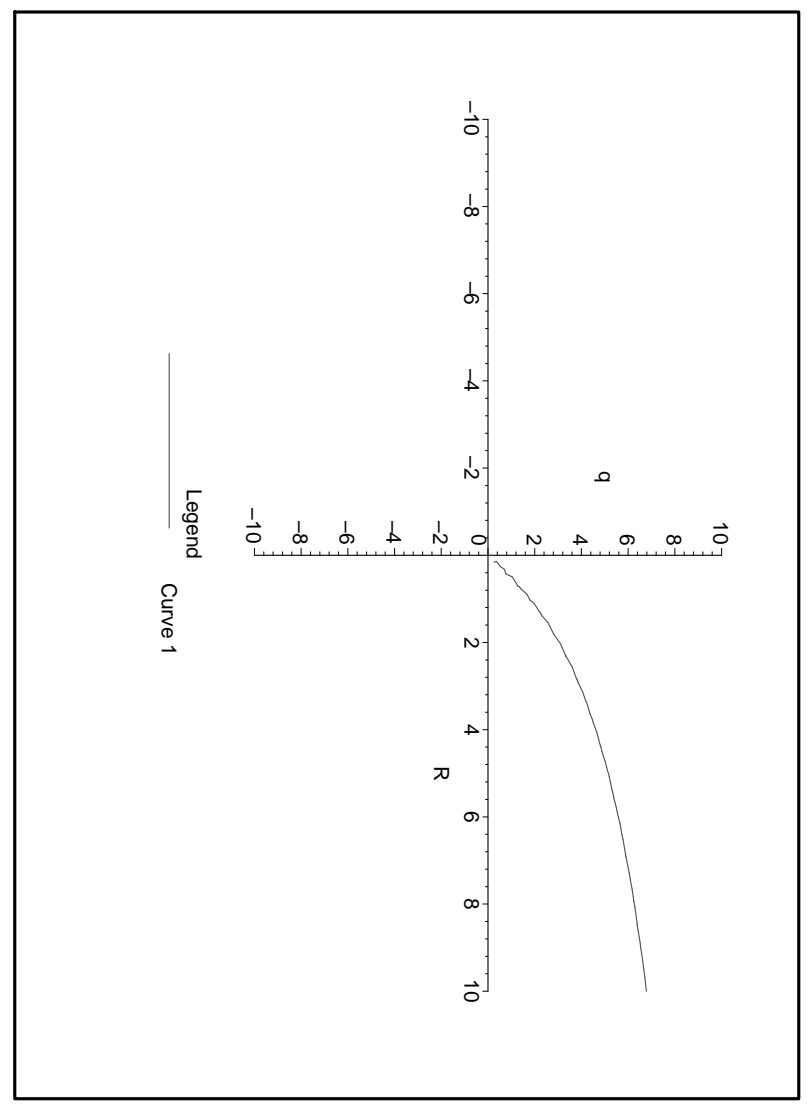

Figure 5: The curvature scalar plotted as a function of $q$, describing the fluctuations in massless non-rotating quarkonia near the Coulambic regime. 


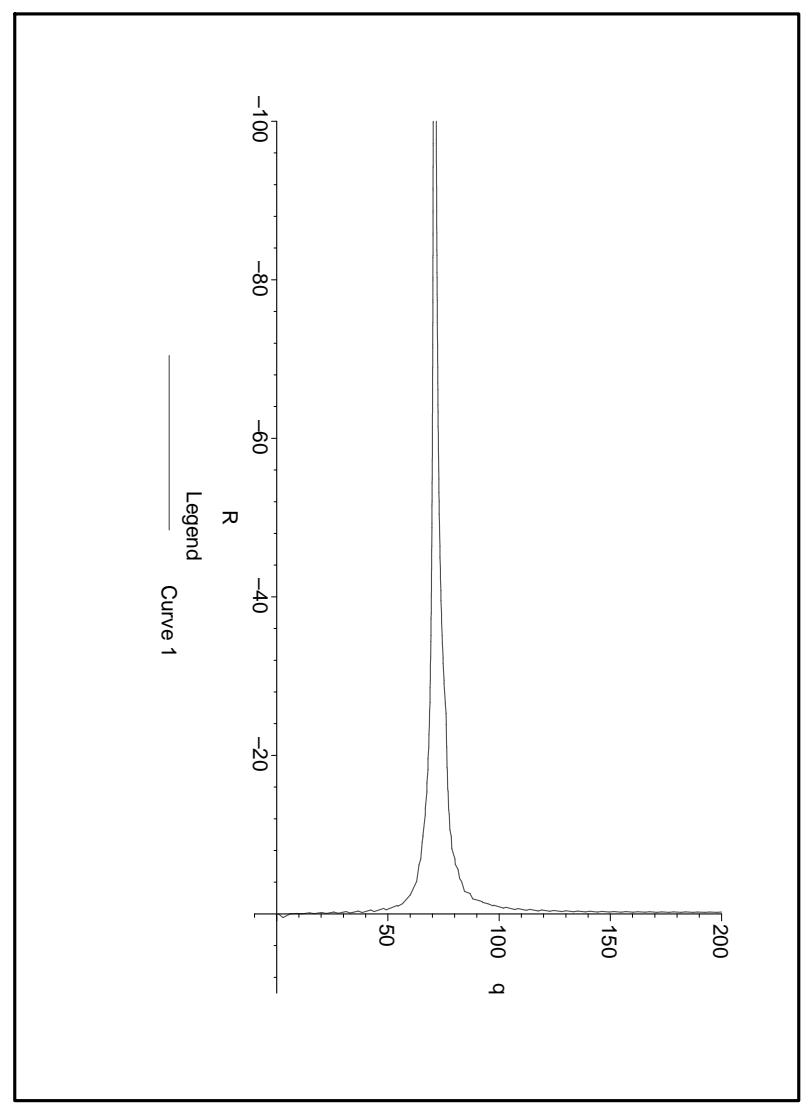

Figure 6: The curvature scalar plotted as a function of $q$, describing the fluctuations in massless non-rotating quarkonia in the rising regime. 
of massless non-rotating quarkonia exists in certain bands. A closer view shows that the limiting Coulambic quarkonia are decaying and interacting particles. For the regime of the rising potential, e.g., $p=0.51$, the respective surface plots of the determinant of the metric tensor and scalar curvature are respectively shown in the Figs. (3) and (6), which shows that the limiting rising potential quarkonia are stable and non-interacting particles. This follows from the fact that the determinant instability is present only for a specific set $q$.

\subsection{Regge Rotating Quarkonia}

In the present subsection, we analyze the nature of massless rotating quarkonia generated by the parameters $q, p$ and angular momentum $J$. Following Eqn.(6), we find that the modified strong QCD coupling takes the form

$$
A(q, p, J)=\frac{1}{b} \frac{p}{\ln \left(1+p(q / L)^{p}\right)}+\frac{1}{b_{1}} J(J+1) .
$$

To focus on the intrinsic geometry of the present case, we chose the parameters $q, p, J$ as the variables for the QCD coupling. As in the previous subsection, we may again exploit the definition of the Hessian function $\operatorname{Hess}(A(q, p, J))$ of the QCD coupling. Considering the analysis of the Regge model, we find that the components of the metric tensor in the framework has the same characterizations for the $g_{q q}, g_{q p}$ and $g_{p p}$ as obtained for the corresponding rotating massless quarkonia. The component of the metric tensor for the rotation parameter turns out to be $g_{J J}=\frac{2}{b_{1}}$. While, the remaining components of the metric tensor, involving $J$ and either the $q$ or $p$, vanish identically.

It follows that the pure pair correlations $\left\{g_{q q}, g_{p p}, J J\right\}$ between the parameters $\{q, p, J\}$ remain positive same as in the case of the non-rotating quarkonia. Further, our computation demonstrates the over-all nature of the parametric fluctuations. In fact, we find that the determinant of the metric tensor reduces to the following simple expression

$$
\|g\|=\frac{2 p^{3}}{b^{2} b_{1} q^{2} l(p)^{5} \exp (3 l(p))} n_{g}^{Q},
$$

where the $n_{g}^{Q}(q, p)$ remains the same as for the non-rotating case. It is worth mentioning that the Regge rotating massless quarkonia is well-behaved, as long as the corresponding rotating massless quarkonia remains so. Over the domain of the parameters $\{q, p, J\}$, we thus observe that the Gaussian fluctuations have the same set of thermodynamic metric stability structures, as long as $b_{1}>0$. The observation of the metric structure shows that the Regge rotating massless quarkonia is stable on the $q p$-surface if the index parameter $p$ and the function $n_{g}^{Q}(q, p)$, appearing in the numerator of the determinant of the metric tensor have the same sign.

Furthermore, we may easily analyze the underlying important conclusions for the specific considerations of Regge rotating massless quarkonia. As in the case of the non-rotating massless case, the global nature of the scalar curvature and associated phase transitions may thus be determined over the range of parameters describing the Regge rotating quarkonia of interest. Since the previous non-rotating configuration is trivially embedded in the Regge rotating configuration, we thus find that the thermodynamic scalar curvature remains exactly the same as for the non-rotating massless quarkonia. The equality of the two scalar curvatures shows that the Regge rotation keeps the same global thermodynamic stability structures as the non-rotating massless counterpart. In the Regge trajectory model, we thus find an interesting conclusion that all possible local and global thermodynamic stability behavior of the massless quarkonia remains the same up to the sign of $b_{1}$, as if there were no rotation in the underlying configuration.

The fact that the efficiency of the rotation induces a mass to the quarkonium is analyzed by considering the Bloch-Nordsieck resummation of the angular phases. As per the analysis of the next subsections, our method offers a non-linear generic characterization for the quarkonium configurations, which we consider to be neither purely massless nor purely rotating, but a strongly coupled QCD.

\section{Massive Quarkonia}

In this section, we extend the intrinsic geometric analysis for realistic quarkonia and compute the associated local and global thermodynamic quantities in the subsequent subsections. Following the notations of the previous 
section, a given generic quarkonia can analytically be easily analyzed about some local equilibrium, if we can fix one of the parameter. The logic simply follows from the fact that the underlying configurations reduce to an intrinsic surface, and thus are easy to compute. Such an assumption is allowed for the specific phases of the QCD, whether one considers the Coulomb phase or the rising phase. Our analysis certainly does not stop here, but we indeed extend it for the general quarkonia with $\{q, p, J\}$ all fluctuating. Let us firstly illustrate the cases for the two parameter configurations with either $\{q, J\}$ or $\{q, m\}$ fluctuating and then systematically for the generic quarkonia.

\subsection{Quarkonia in QJ-plane}

In this subsection, we shall use the essential features of thermodynamic geometry, thus developed, to describe the quarkonia using modified strong QCD coupling, with an increasing number of parameters. Let us focus our attention on the geometric nature of the local and global correlations in the neighbourhood of small fluctuations, in the chosen quarkonium configurations. As per the consideration of massive quarkonia, the associated resummed strong QCD coupling is given by

$$
A(q, J):=\frac{1}{b} \frac{p}{\ln \left(1+p(q / L)^{p}\right)} \ln \left(\frac{\sqrt{J}+\sqrt{J-q}}{\sqrt{J}-\sqrt{J-q}}\right)(1-J(0, a \sqrt{q})),
$$

where $J(\nu, x)$ is the Bessel function of the first kind of the order $\nu$. As stated earlier, the thermodynamic metric in the parameter space is given by the Hessian matrix $\operatorname{Hess}(A(q, J))$ of the strong QCD coupling with

respect to the variables defining the thermodynamic manifolds. In order to simplify the subsequent expressions, let us define the logarithmic factor of the concerned Bloch-Nordsieck rotation as

$$
f(q, J):=\ln \left(\frac{\sqrt{J}+\sqrt{J-q}}{\sqrt{J}-\sqrt{J-q}}\right) .
$$

In this framework, it turns out that the fluctuation nature of parametric pair correlations may be easily divulged in terms of the momentum transfer and rotation parameter of the underlying quarkonia. Following Eqn.(10), we find, under the Gaussian fluctuations of $\{q, J\}$, that the components of the metric tensor are

$$
\begin{aligned}
g_{q q} & =-\frac{p}{4 b l(p)^{3} \exp (2 l(p)) q^{5 / 2}(J-q)^{3 / 2}}\left(n_{11}^{(0) J}+n_{11}^{(1) J} l(p)+n_{11}^{(2) J} l(p)^{2}\right), \\
g_{q p} & =\frac{p}{2 b l(p)^{2} \exp (l(p)) q^{3 / 2} \sqrt{J}(J-q)^{3 / 2}}\left(n_{12}^{(0) J}+n_{12}^{(1) J} l(p)\right), \\
g_{p p} & =-\frac{p}{2 b l(p) J^{3 / 2}(J-q)^{3 / 2}}(2 J-q)(1-J(0, a \sqrt{q})) .
\end{aligned}
$$

As a result, we find without any approximation that the factors in the numerator of the local pair correlation associated with the $q q$-components are expressed as

$$
\begin{aligned}
n_{11}^{(0) J}= & 8 p^{4} f\left((q / L)^{p}\right)^{2} \sqrt{J-q}\left(q^{3 / 2}-q^{3 / 2} J(0, a \sqrt{q})-\sqrt{q} J+\sqrt{q} J(0, a \sqrt{q}) J\right), \\
n_{11}^{(1) J}= & -4 p^{2} f \sqrt{J-q}(q / L)^{p}\left(J(1, a \sqrt{q}) a q^{2}+q^{3 / 2}-q^{(3 / 2} J(0, a \sqrt{q})+J(1, a \sqrt{q}) a q J\right. \\
& -\sqrt{q} J+\sqrt{q} J(0, a \sqrt{q}) J)+8 p^{2}(q / L)^{p}\left(\sqrt{J} q^{3 / 2}+J^{3 / 2} \sqrt{q} J(0, a \sqrt{q})\right. \\
& \left.-\sqrt{J} q^{3 / 2} J(0, a \sqrt{q})-J^{3 / 2} \sqrt{q}\right)+4 p^{3} f \sqrt{J-q}(q / L)^{p}(\sqrt{q} J-\sqrt{q} J(0, a \sqrt{q}) J \\
& \left.+q^{3 / 2} J(0, a \sqrt{q})-q^{3 / 2}\right)+4 p^{3} f \sqrt{J-q}\left((q / L)^{p}\right)^{2}\left(q^{3 / 2}+\sqrt{q} J(0, a \sqrt{q}) J\right. \\
& \left.-q^{3 / 2} J(0, a \sqrt{q})+J(1, a \sqrt{q}) a q J-\sqrt{q} J-J(1, a \sqrt{q}) a q^{2}\right) \\
& +8 p^{3}\left((q / L)^{p}\right)^{2}\left(\sqrt{J} q^{3 / 2}-\sqrt{J} q^{3 / 2} J(0, a \sqrt{q})+J^{3 / 2} \sqrt{q} J(0, a \sqrt{q})-J^{3 / 2} \sqrt{q}\right), \\
n_{11}^{(2) J}= & 2 \sqrt{J}\left(6 q^{3 / 2} p(q / L)^{p}+3 q^{3 / 2}-4 J^{3 / 2} \sqrt{q}-3 q^{3 / 2} J(0, a \sqrt{q})-2 J(1, a \sqrt{q}) a q^{2}\right) \\
& +f \sqrt{J-q}\left(J(0, a \sqrt{q}) a^{2} q^{5 / 2}-J(0, a \sqrt{q}) a^{2} q^{3 / 2} J+2 J(1, a \sqrt{q}) a q J\right. \\
& \left.-2 J(1, a \sqrt{q}) a q^{2}\right)+4 J^{3 / 2}(J(1, a \sqrt{q}) a q+\sqrt{q} J(0, a \sqrt{q}))
\end{aligned}
$$




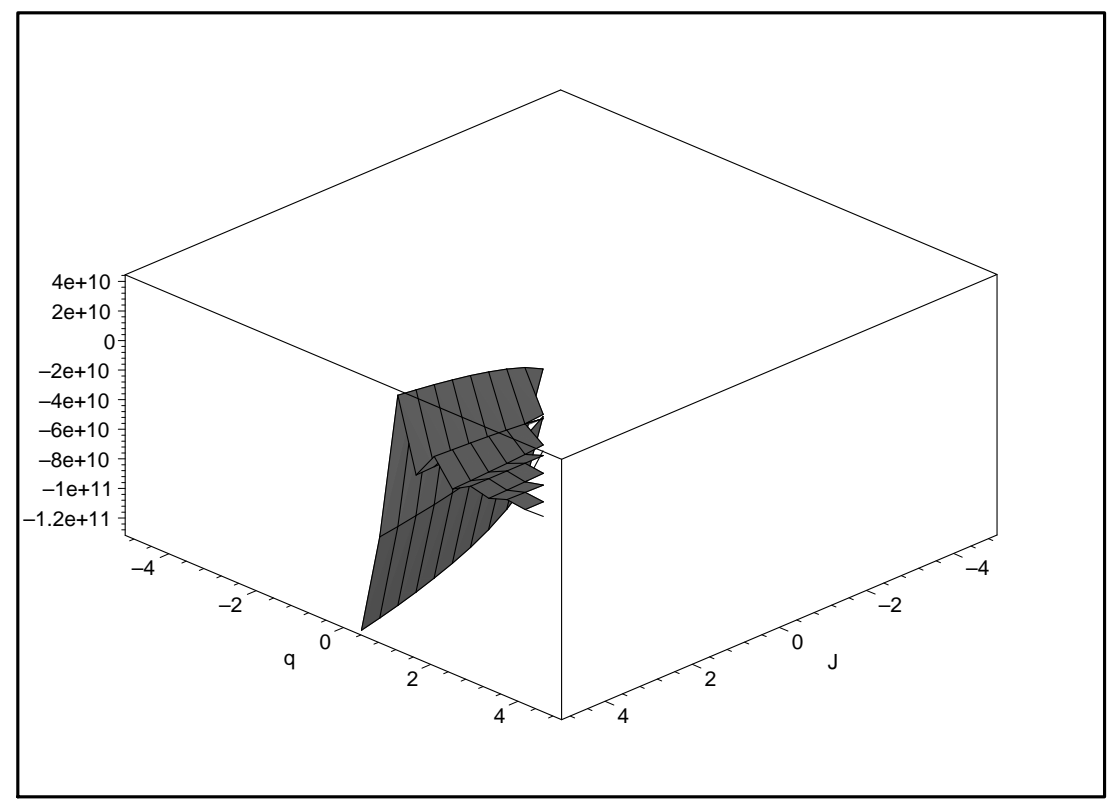

Figure 7: The $q q$-component of the metric tensor plotted as a function of $q$ and the angular momentum $J$, describing the heat capacity of the charge in massive rotating quarkonia.

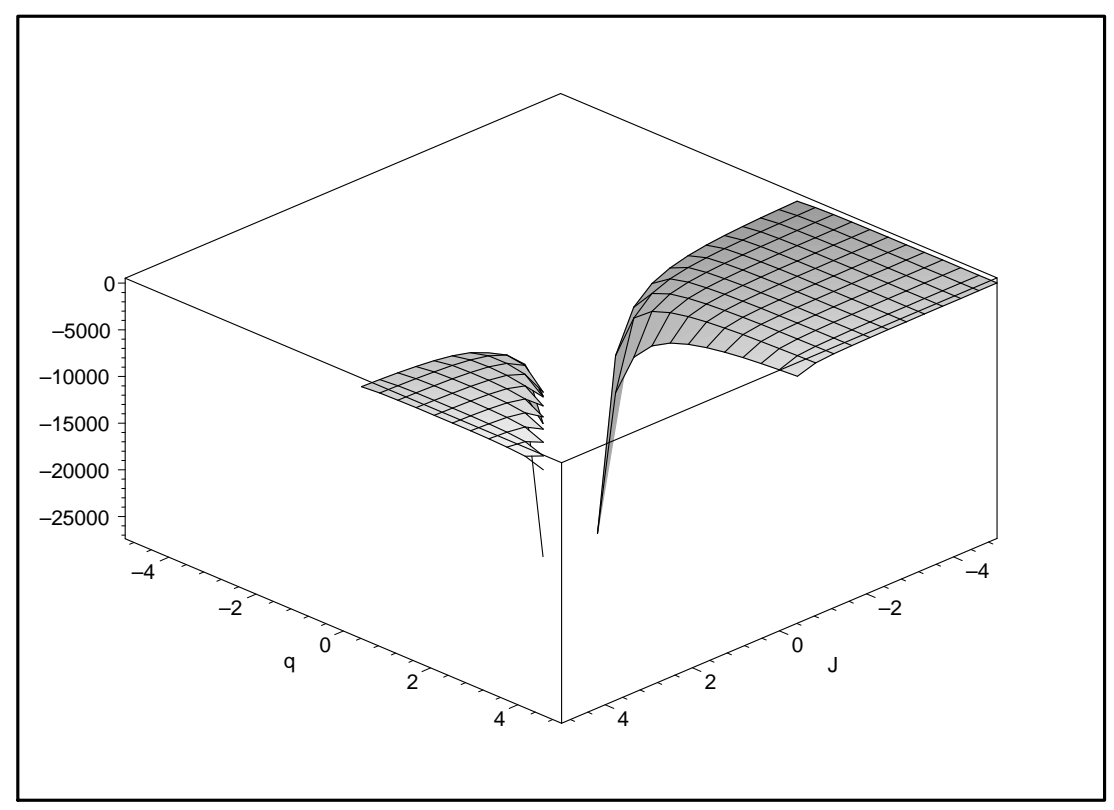

Figure 8: The $J J$-component of the metric tensor plotted as a function of $q$ and the angular momentum $J$, describing the heat capacity of the angular momentum in massive rotating quarkonia. 


$$
\begin{aligned}
& +2 p(q / L)^{p}\left(f a^{2} q^{5 / 2} \sqrt{J-q} J(0, a \sqrt{q})-f a^{2} q^{3 / 2} \sqrt{J-q} J(0, a \sqrt{q}) J\right. \\
& -2 f J(1, a \sqrt{q}) a q^{2} \sqrt{J-q}+2 f J(1, a \sqrt{q}) a q \sqrt{J-q} J+4 J^{3 / 2} J(1, a \sqrt{q}) a q \\
& \left.-4 J^{3 / 2} \sqrt{q}+4 J^{3 / 2} \sqrt{q} J(0, a \sqrt{q})-4 \sqrt{J} J(1, a \sqrt{q}) a q^{2}-6 \sqrt{J} q^{3 / 2} J(0, a \sqrt{q})\right) \\
& +p^{2}\left((q / L)^{p}\right)^{2}\left(f a^{2} q^{5 / 2} \sqrt{J-q} J(0, a \sqrt{q})-f a^{2} q^{3 / 2} \sqrt{J-q} J(0, a \sqrt{q}) J\right. \\
& +2 f J(1, a \sqrt{q}) a q \sqrt{J-q} J-2 f J(1, a \sqrt{q}) a q^{2} \sqrt{J-q}-4 \sqrt{J} J(1, a \sqrt{q}) a q^{2} \\
& +4 J^{3 / 2} J(1, a \sqrt{q}) a q+4 J^{3 / 2} \sqrt{q} J(0, a \sqrt{q})-4 J^{3 / 2} \sqrt{q}+6 \sqrt{J} q^{3 / 2} \\
& \left.-6 \sqrt{J} q^{3 / 2} J(0, a \sqrt{q})\right) .
\end{aligned}
$$

While, the factors in the numerator of the associated $q J$-component are given by

$$
\begin{aligned}
n_{12}^{(0) J}= & 2 p^{2}(q / L)^{p}\left(q^{3 / 2}-J^{3 / 2}+J(0, a \sqrt{J})-J(0, a \sqrt{J})\right), \\
n_{12}^{(1) J}= & q^{3 / 2}+a q J J(1, a \sqrt{J})-a q^{2} J(1, a \sqrt{J})-q^{3 / 2} J(0, a \sqrt{J}) \\
& +(q / L)^{p} p\left(q^{3 / 2}+a q J J(1, a \sqrt{J})-a q^{2} J(1, a \sqrt{J})-q^{3 / 2} J(0, a \sqrt{J})\right) .
\end{aligned}
$$

Herewith, we see that the geometric nature of parametric pair correlations turns out to be remarkably interesting. The fluctuating quarkonia may be easily described in terms of the $q$ and $J$. For the configurations with the same sign of the index $p$ and constant $b$, it is evident that the principle components of the metric tensor, signifying self pair correlations, are positive definite functions in a non-trivial range $q$. The local stability requires that (i) $q q$ - fluctuations satisfy the constraint

$$
n_{11}^{(0) J}+n_{11}^{(1) J} l(p)+n_{11}^{(2) J} l(p)^{2}<0
$$

and (ii) $J J$ - fluctuations be constrained to the following limiting values of the Bessel function

$$
\begin{aligned}
J(0, a \sqrt{q}) & <1, q>2 J \\
& >1, \quad q<2 J
\end{aligned}
$$

A straightforward computation demonstrates the over-all nature of the parametric fluctuations. In this case, we find that the determinant of the metric tensor reduces to the following expression

$$
g=-\frac{p^{2}}{8 b^{2} l(p)^{4} \exp (2 l(p)) q^{5 / 2} J^{3 / 2}(J-q)^{3 / 2}}\left(n_{g}^{(0) J}+n_{g}^{(1) J} l(p)+n_{g}^{(2) J} l(p)^{2}\right),
$$

where the coefficients $\left\{n_{g}^{(1) J}, n_{g}^{(2) J}\right\}$ appearing in the determinant of the metric tensor factorize as

$$
\begin{aligned}
n_{g}^{(1) J} & =4 p^{2}(q / L)^{p}\left(n_{g}^{(12) J}+n_{g}^{(13) J} p(q / L)^{p}\right), \\
n_{g}^{(2) J} & =n_{g}^{(20) J}+2 n_{g}^{(21) J} p(q / L)^{p}+n_{g}^{(23) J}\left((q / L)^{p}\right)^{2} p^{2} .
\end{aligned}
$$

Explicitly, we find that all factors of the numerator of the determinant can be presented as

$$
\begin{aligned}
n_{g}^{(0) J}= & 8 p^{4}\left((q / L)^{p}\right)^{2}\left(\sqrt{J} \sqrt{q} \sqrt{J-q}+\sqrt{J} \sqrt{q} \sqrt{J-q} J(0, a \sqrt{q})^{2}\right. \\
& -f q^{3 / 2} J(0, a \sqrt{q})^{2}-f q^{3 / 2}+2 J f \sqrt{q}+2 f q^{3 / 2} J(0, a \sqrt{q}) \\
& \left.-2 \sqrt{J} \sqrt{q} \sqrt{J-q} J(0, a \sqrt{q})+2 J f \sqrt{q} J(0, a \sqrt{q})^{2}-4 J f \sqrt{q} J(0, a \sqrt{q})\right), \\
n_{g}^{(12) J}= & \left(f J(1, a \sqrt{q}) a q^{2}-f q^{3 / 2}-f J(1, a \sqrt{q}) a q^{2} J(0, a \sqrt{q})-f q^{3 / 2} J(0, a \sqrt{q})^{2}\right. \\
& +2 f q^{3 / 2} J(0, a \sqrt{q})+2 \sqrt{J} q J(1, a \sqrt{q}) a \sqrt{J-q} J(0, a \sqrt{q}) \\
& +2 J f \sqrt{q}+2 J f J(1, a \sqrt{q}) a q J(0, a \sqrt{q})+2 J f \sqrt{q} J(0, a \sqrt{q})^{2} \\
& -2 J f J(1, a \sqrt{q}) a q-2 \sqrt{J} q J(1, a \sqrt{q}) a \sqrt{J-q}+4 \sqrt{J} \sqrt{q} \sqrt{J-q} \\
& \left.+4 \sqrt{J} \sqrt{q} \sqrt{J-q} J(0, a \sqrt{q})^{2}-4 J f \sqrt{q} J(0, a \sqrt{q})-8 \sqrt{J} \sqrt{q} \sqrt{J-q} J(0, a \sqrt{q})\right),
\end{aligned}
$$




$$
\begin{aligned}
& n_{g}^{(13) J}=f\left(q^{3 / 2}+q^{3 / 2} J(0, a \sqrt{q})^{2}-2 J \sqrt{q} J(0, a \sqrt{q})^{2}-2 J \sqrt{q}\right. \\
& \left.-2 q^{3 / 2} J(0, a \sqrt{q})+4 J \sqrt{q} J(0, a \sqrt{q})\right)+\left((q / L)^{p}\right)\left(f J(1, a \sqrt{q}) a q^{2}\right. \\
& -f q^{3 / 2} J(0, a \sqrt{q})^{2}-f J(1, a \sqrt{q}) a q^{2} J(0, a \sqrt{q})-f q^{3 / 2} \\
& +2 f J \sqrt{q}-2 \sqrt{J} q J(1, a \sqrt{q}) a \sqrt{J-q}+2 f q^{3 / 2} J(0, a \sqrt{q}) \\
& +2 f J J(1, a \sqrt{q}) a q J(0, a \sqrt{q})-2 f J J(1, a \sqrt{q}) a q+2 f J \sqrt{q} J(0, a \sqrt{q})^{2} \\
& +2 \sqrt{J} q J(1, a \sqrt{q}) a \sqrt{J-q} J(0, a \sqrt{q})+4 \sqrt{J} \sqrt{q} \sqrt{J-q} J(0, a \sqrt{q})^{2} \\
& +4 \sqrt{J} \sqrt{q} \sqrt{J-q}-4 J f \sqrt{q} J(0, a \sqrt{q})-8 \sqrt{J} \sqrt{q} \sqrt{J-q} J(0, a \sqrt{q})), \\
& n_{g}^{(20) J}=2 \sqrt{J}\left(q^{3 / 2} J(1, a \sqrt{q})^{2} a^{2} \sqrt{J-q}+4 \sqrt{q} \sqrt{J-q}+4 \sqrt{q} \sqrt{J-q} J(0, a \sqrt{q})^{2}\right. \\
& +4 J(1, a \sqrt{q}) a q \sqrt{J-q} J(0, a \sqrt{q})-4 J(1, a \sqrt{q}) a q \sqrt{J-q} \\
& -8 \sqrt{q} \sqrt{J-q} J(0, a \sqrt{q}))+f\left(a^{2} q^{5 / 2} J(0, a \sqrt{q})^{2}-a^{2} q^{5 / 2} J(0, a \sqrt{q})\right. \\
& +2 a q^{2} J(1, a \sqrt{q})-2 J(1, a \sqrt{q}) a q^{2} J(0, a \sqrt{q})+2 J a^{2} q^{3 / 2} J(0, a \sqrt{q}) \\
& \left.-2 J a^{2} q^{3 / 2} J(0, a \sqrt{q})^{2}-4 J a q J(1, a \sqrt{q})+4 J J(1, a \sqrt{q}) a q J(0, a \sqrt{q})\right), \\
& n_{g}^{(21) J}=\left(f a^{2} q^{5 / 2} J(0, a \sqrt{q})^{2}-f a^{2} q^{5 / 2} J(0, a \sqrt{q})-2 f J(1, a \sqrt{q}) a q^{2} J(0, a \sqrt{q})\right. \\
& +2 f a q^{2} J(1, a \sqrt{q})+2 \sqrt{J} q^{3 / 2} J(1, a \sqrt{q})^{2} a^{2} \sqrt{J-q}+2 J f a^{2} q^{3 / 2} J(0, a \sqrt{q}) \\
& -2 J f a^{2} q^{3 / 2} J(0, a \sqrt{q})^{2}+4 J f J(1, a \sqrt{q}) a q J(0, a \sqrt{q})-4 J f a q J(1, a \sqrt{q}) \\
& +8 \sqrt{J} \sqrt{q} \sqrt{J-q} J(0, a \sqrt{q})^{2}-8 \sqrt{J} J(1, a \sqrt{q}) a q \sqrt{J-q}+8 \sqrt{J} \sqrt{q} \sqrt{J-q} \\
& +8 \sqrt{J} J(1, a \sqrt{q}) a q \sqrt{J-q} J(0, a \sqrt{q})-16 \sqrt{J} \sqrt{q} \sqrt{J-q} J(0, a \sqrt{q})), \\
& n_{g}^{(23) J}=f a^{2} q^{5 / 2} J(0, a \sqrt{q})^{2}-f a^{2} q^{5 / 2} J(0, a \sqrt{q})+2 f J(1, a \sqrt{q}) a q^{2} \\
& -2 f a q^{2} J(1, a \sqrt{q}) J(0, a \sqrt{q})+2 \sqrt{J} q^{3 / 2} J(1, a \sqrt{q})^{2} a^{2} \sqrt{J-q} \\
& +2 J f a^{2} q^{3 / 2} J(0, a \sqrt{q})-2 J f a^{2} q^{3 / 2} J(0, a \sqrt{q})^{2} \\
& +4 J f a q J(1, a \sqrt{q}) J(0, a \sqrt{q})-4 J f a q J(1, a \sqrt{q}) \\
& -8 \sqrt{J} J(1, a \sqrt{q}) a q \sqrt{J-q}+8 \sqrt{J} \sqrt{q} \sqrt{J-q} J(0, a \sqrt{q})^{2} \\
& +8 \sqrt{J} J(1, a \sqrt{q}) a q \sqrt{J-q} J(0, a \sqrt{q})+8 \sqrt{J} \sqrt{q} \sqrt{J-q} \\
& -16 \sqrt{J} \sqrt{q} \sqrt{J-q} J(0, a \sqrt{q}) .
\end{aligned}
$$

From Figs.(7) and (8), we see how the pure components of the metric tensor behave, when they are plotted against $q$ and the angular momentum. For the choice $L=22500, p=5 / 6, b=1$ and $a=100000$, the $q q$-heat capacity as depicted in the Fig.(7) shows a large variation of the minima and maxima, which respectively take in an order of the amplitude $+10^{10}$ and $-10^{11}$ and occur for $q, J \in(0,4)$. In this case, we further observe for all $J \in(0,4)$ that the underlying quarkonia become highly unstable as $q$ positively approaches the origin.

On the other hand, the $J J$-heat capacity in Fig.(8) shows only negative variation of order $-10^{5}$, which occurs when $q, J$ tend to the origin. In this case, we notice that all local interactions are present in the region $q \in(0,4)$ and $J \in(-4,4)$. The strength of these local interactions depends on the domain chosen in the $(q, J)$ space. These fluctuations signify the thermodynamical interactions present in the strongly coupled quarkonia.

The corresponding plot for the determinant of the metric tensor is depicted in Fig.(9). For the above choice of the parameters, the Fig.(91) shows the graphical nature of the determinant of the metric tensor. These plots explicate the thermodynamically (un)stability regions for the underlying massive quarkonia lying in a given QCD phase. Combining the effects of all fluctuations of the $\{q, J\}$, we observe that the quarkonia are stable for $q:=Q^{2} \in(1,4)$. In general, the global stability requires that the determinant of the metric tensor must be positive definite, which in the present case transform as

$$
n_{g}^{J}:=n_{g}^{(0) J}+n_{g}^{(1) J} l(p)+n_{g}^{(2) J} l(p)^{2}<0 .
$$

In this case, it turns out that the thermodynamic curvature may be written as the series of the charmonium logarithmic factor $l(p)$ and Bloch-Nordsieck logarithmic factor $f(q, J)$ of rotation as the coefficient of the expansion. Systematically, the exact expression for the scalar curvature takes the form

$$
R(q, J)=\frac{b l(p)}{2 p^{2}\left(n_{g}^{J}\right)^{2}} \sum_{n} B_{n} \times(l(p))^{n}
$$




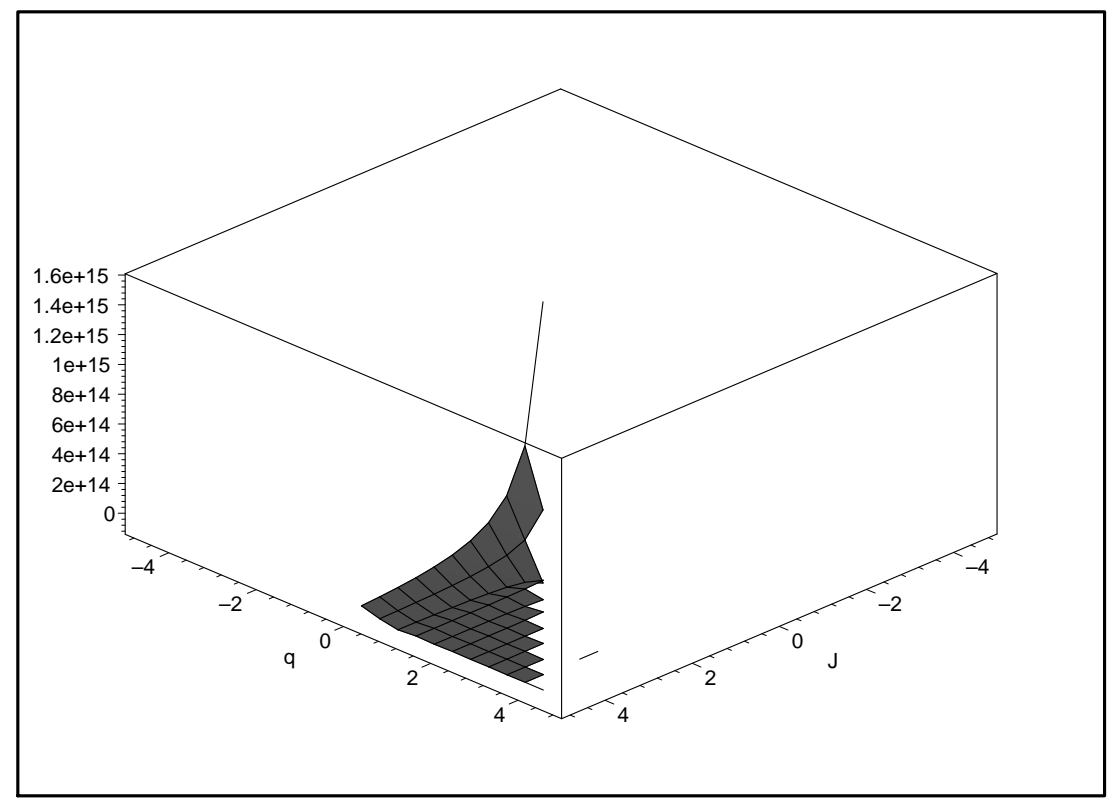

Figure 9: The determinant of the metric tensor plotted as a function of $q$ and the angular momentum $J$, describing the fluctuations in massive rotating quarkonia.

where the $B_{n}$ in the numerator of the scalar curvature are polynomials in $p$, whose coefficients are the functions of the Bloch-Nordsieck logarithmic factor $f(q, J)$. While, the denominator of the scalar curvature precisely takes the numerator of the determinant of the metric tensor as its square. The quantitative properties of the scalar curvature and the Riemann curvature tensor remain similar, as we shall discuss in the next subsection.

\subsection{Quarkonia in QM-plane}

In the present section, we analyze the nature of the fluctuating quarkonia generated by the momentum $Q$, and mass $m$. To focus on the general case, we choose the variable $Q$ as the transverse momentum with the understanding that $k=k_{\perp}$ and the mass considered as an arbitrary real parameter of the system. Following the convention of Bloch-Nordsieck resummation, the strong QCD coupling can be expressed as

$$
A(k, m)=\frac{1}{b} \frac{p}{\ln \left(1+p\left(k^{2} / L\right)^{p}\right)} \ln \left(\frac{m+\sqrt{m^{2}-k^{2}}}{m-\sqrt{m^{2}-k^{2}}}\right)(1-J(0, a k)) .
$$

When the Bloch-Nordsieck resummed strong QCD coupling $A(k, m)$ is allowed to fluctuate as a function of the $\{k, m\}$, we may again exploit the definition of the Hessian function $\operatorname{Hess}(A(k, m))$. Herewith, we find that the components of the metric tensor are given by

$$
\begin{aligned}
g_{k k} & =\frac{p}{b l(p)^{3} \exp \left(2(l(p)) k^{6}\left(m^{2}-k^{2}\right)^{3 / 2}\right.}\left(n_{11}^{(0) M}+n_{11}^{(1) M} l(p)+n_{11}^{(2) M} l(p)^{2}\right), \\
g_{k m} & =\frac{2 p}{b l(p)^{2} \exp (l(p)) k\left(m^{2}-k^{2}\right)^{3 / 2}}\left(n_{12}^{(0) M}+n_{12}^{(1) M} l(p)\right), \\
g_{k m} & =\frac{2 m p}{b l(p)\left(m^{2}-k^{2}\right)^{3 / 2}}(-1+J(0, a k)),
\end{aligned}
$$

where the coefficients $\left\{n_{g}^{(1) M}, n_{g}^{(2) M}\right\}$ appearing in the determinant of the metric tensor factorize as

$$
\begin{aligned}
& n_{11}^{(1) M}=2\left(k^{2} / L\right)^{p} p^{2}\left(n_{11}^{(12) M}+2 n_{11}^{(13) M} p\left(k^{2} / L\right)^{p}\right), \\
& n_{11}^{(2) M}=n_{11}^{(20) M}+2 p n_{11}^{(21) M}\left(k^{2} / L\right)^{p}+p^{2} n_{11}^{(22) M}\left(k^{2} / L\right)^{2 p} .
\end{aligned}
$$




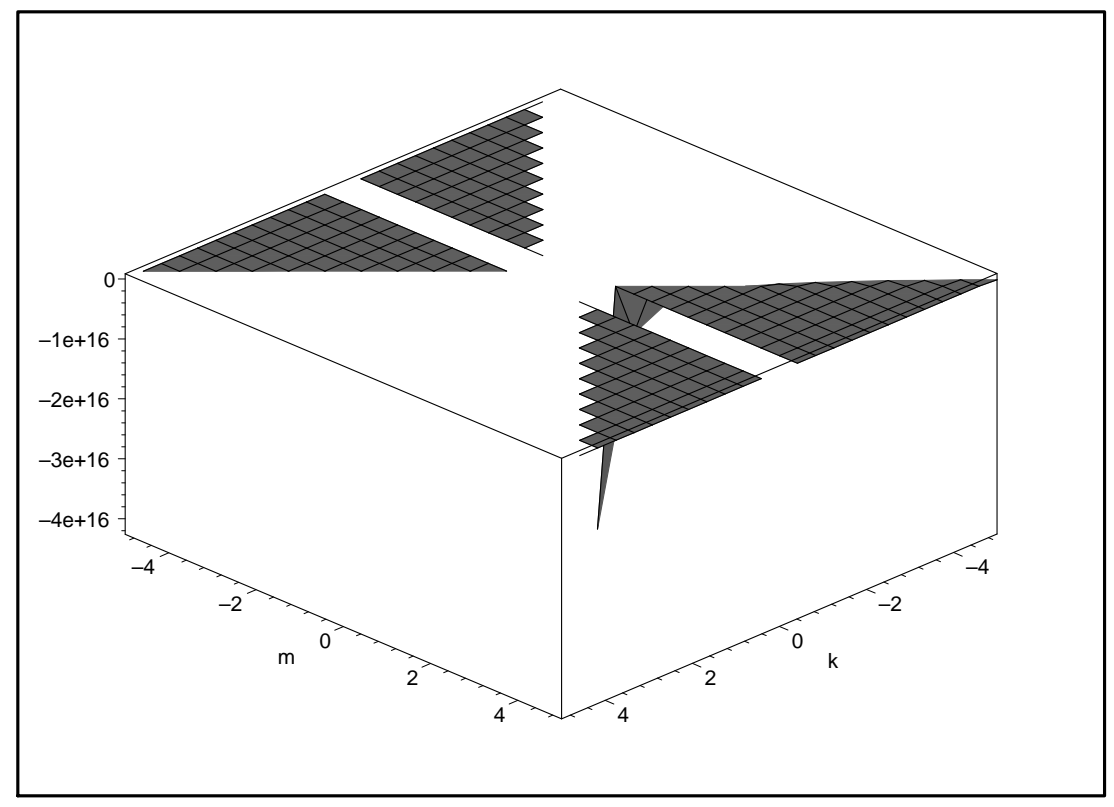

Figure 10: The $k k$-component of the metric tensor plotted as a function of the transverse momentum and mass $k, m$, describing the heat capacity of transverse momentum for the massive rotating quarkonia.

As it might be expected, we find without any approximation that the factors in the numerator of the local pair correlation associated with the $k k$-components can, as before, be easily expressed as

$$
\begin{aligned}
& n_{11}^{(0) M}=8 p^{4} f\left(\left(k^{2} / L\right)^{p}\right)^{2} \sqrt{m^{2}-k^{2}}\left(m^{2}-k^{2}+J(0, a k) k^{2}-J(0, a k) m^{2}\right), \\
& n_{11}^{(12) M}=f \sqrt{m^{2}-k^{2}} J(0, a k) k^{2}-f \sqrt{m^{2}-k^{2}} J(0, a k) m^{2}+4 m^{3}-4 m^{3} J(0, a k) \\
& +4 m k^{2} J(0, a k)+f \sqrt{m^{2}-k^{2}} m^{2}-f \sqrt{m^{2}-k^{2}} k^{2}-4 m k^{2} \\
& -2 f J(1, a k) a k \sqrt{m^{2}-k^{2}} m^{2}+2 f J(1, a k) a k^{3} \sqrt{m^{2}-k^{2}}, \\
& n_{11}^{(13) M}=f \sqrt{m^{2}-k^{2}} k^{2}+f\left(\left(k^{2} / L\right)^{p}\right)^{2} \sqrt{m^{2}-k^{2}} m^{2}-f\left(\left(k^{2} / L\right)^{p}\right)^{2} \sqrt{m^{2}-k^{2}} k^{2} \\
& -f \sqrt{m^{2}-k^{2}} J(0, a k) k^{2}-f \sqrt{m^{2}-k^{2}} m^{2}+f \sqrt{m^{2}-k^{2}} J(0, a k) m^{2} \\
& -f\left(\left(k^{2} / L\right)^{p}\right)^{2} \sqrt{m^{2}-k^{2}} J(0, a k) m^{2}+f\left(\left(k^{2} / L\right)^{p}\right)^{2} \sqrt{m^{2}-k^{2}} J(0, a k) k^{2} \\
& -2 f J(1, a k) a\left(\left(k^{2} / L\right)^{p}\right)^{2} k \sqrt{m^{2}-k^{2}} m^{2}+2 f J(1, a k) a\left(\left(k^{2} / L\right)^{p}\right)^{2} k^{3} \sqrt{m^{2}-k^{2}} \\
& +4 m k^{2}\left(\left(k^{2} / L\right)^{p}\right)^{2} J(0, a k)-4 m^{3}\left(\left(k^{2} / L\right)^{p}\right)^{2} J(0, a k)+4 m^{3}\left(\left(k^{2} / L\right)^{p}\right)^{2} \\
& -4 m k^{2}\left(\left(k^{2} / L\right)^{p}\right)^{2} \\
& n_{11}^{(20) M}=f a k^{3} \sqrt{m^{2}-k^{2}} J(1, a k)-f a k \sqrt{m^{2}-k^{2}} J(1, a k) m^{2}+f a^{2} k^{2} \sqrt{m^{2}-k^{2}} J(0, a k) m^{2} \\
& -f a^{2} k^{4} \sqrt{m^{2}-k^{2}} J(0, a k)+2 m^{3}+4 m k^{2} J(0, a k)-4 m^{3} k J(1, a k) a-4 m k^{2} \\
& -2 m^{3} l(p)^{2} J(0, a k)+4 m k^{3} J(1, a k) a, \\
& n_{11}^{(21) M}=f a k^{3} \sqrt{m^{2}-k^{2}} J(1, a k)-f a^{2} k^{4} \sqrt{m^{2}-k^{2}} J(0, a k)-f a k \sqrt{m^{2}-k^{2}} J(1, a k) m^{2} \\
& +f a^{2} k^{2} \sqrt{m^{2}-k^{2}} J(0, a k) m^{2}-2 m^{3} J(0, a k)+2 m^{3}-4 m^{3} k J(1, a k) a p-4 m k^{2} \\
& +4 m k^{3} J(1, a k) a+4 m k^{2} J(0, a k), \\
& n_{11}^{(22) M}=f a k^{3} \sqrt{m^{2}-k^{2}} J(1, a k)-f a^{2} k^{4} \sqrt{m^{2}-k^{2}} J(0, a k)+f a^{2} k^{2} \sqrt{m^{2}-k^{2}} J(0, a k) m^{2} \\
& -f a k \sqrt{m^{2}-k^{2}} J(1, a k) m^{2}+2 m^{3}-2 m^{3} J(0, a k)-4 m^{3} k J(1, a k) a \\
& -4 m k^{2}+4 m k^{2} J(0, a k)+4 m k^{3} J(1, a k) a \text {. }
\end{aligned}
$$

In the present case, the factors of the numerator of the $\mathrm{km}$-component of the metric tensor are 


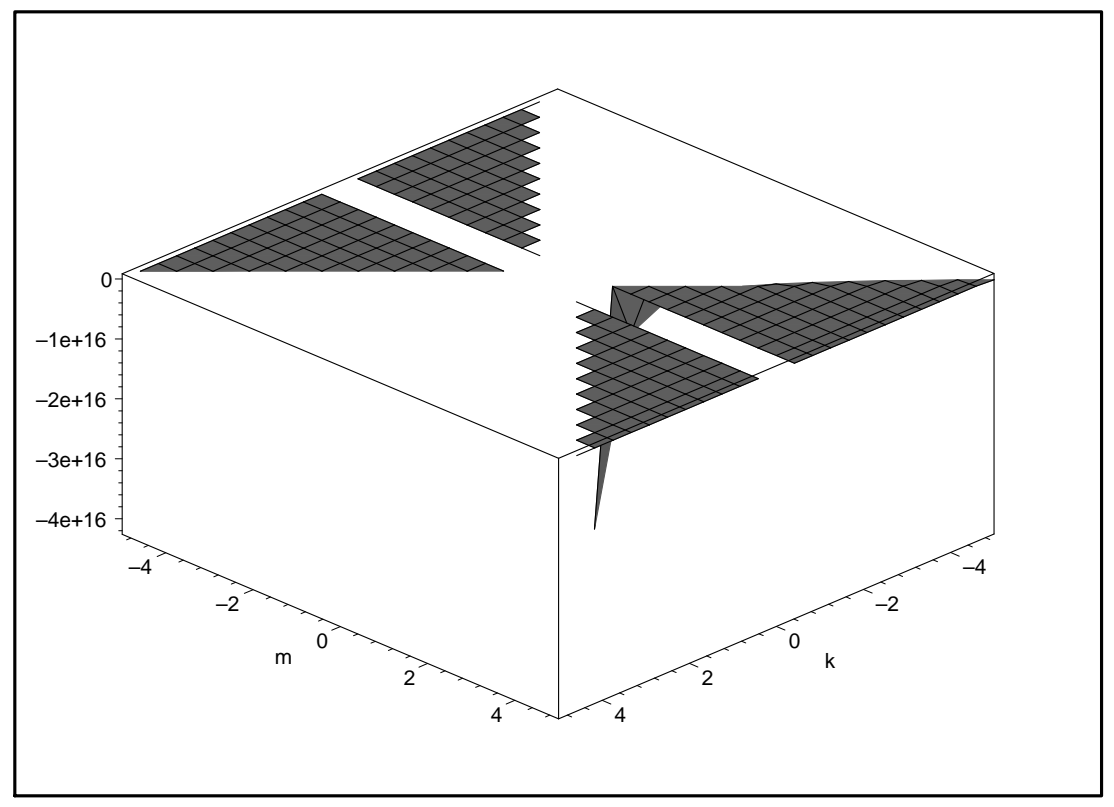

Figure 11: The $m m$-component of the metric tensor plotted as a function of the transverse momentum and mass $k, m$, describing the heat capacity of mass for the massive rotating quarkonia.

$$
\begin{aligned}
n_{12}^{(0) M}= & 2 p^{2}\left(k^{2} / L\right)^{p}\left(k^{2}+J(0, a k) m^{2}-J(0, a k) k^{2}-m^{2}\right), \\
n_{12}^{(1) M}= & k^{2}-k^{2} J(0, a k)+J(1, a k) a k m^{2}-J(1, a k) a k^{3} \\
& +J(1, a k) a k m^{2} p\left(k^{2} / L\right)^{p}-J(1, a k) a k^{3} p\left(k^{2} / L\right)^{p} \\
& -k^{2} J(0, a k) p\left(k^{2} / L\right)^{p}+k^{2} p\left(k^{2} / L\right)^{p} .
\end{aligned}
$$

When the fluctuations of the quarkonia are described in terms of the transverse momentum $k$ and mass $m$, we observe that the principle components of the metric tensor, signifying self pair correlations, are positive definite functions in a non-trivial range of $k$. For the configurations with the same sign of the index $p$ and constant $b$, it turns out that the local stability requires that (i) $q q$ - fluctuations satisfy the constraint

$$
n_{11}^{(0) M}+n_{11}^{(1) M} l(p)+n_{11}^{(2) M} l(p)^{2}>0,
$$

and (ii) $m m$ - fluctuations pose a constraint on the Bessel function such that the underlying transverse momentum of the configuration must be limited to the condition of $J(0, a k)>1$.

The determinant of the metric tensor turns out to be in a similar rational form, as it was obtained in the previous subsection. However, the simplifications are relatively straightforward, which in turn implies the following compact expression for the determinant of the metric tensor

$$
g=-\frac{2 p^{2}}{b^{2} l(p)^{4} \exp \left(l(p)^{2}\right) k^{2}\left(m^{2}-k^{2}\right)^{3 / 2}}\left(n_{g}^{(0) M}+n_{g}^{(1) M} l(p)+n_{g}^{(2) M} l(p)^{2}\right),
$$

with the following factorization

$$
\begin{aligned}
& n_{g}^{(1) M}=2 p^{2}\left(k^{2} / L\right)^{p}\left(\left(n_{g}^{(12) M}+p\left(\left(k^{2} / L\right)^{p}\right) n_{g}^{(13) M}\right),\right. \\
& n_{g}^{(2) M}=n_{g}^{(20) M}+2 n_{g}^{(21) M} p\left(k^{2} / L\right)^{p}+n_{g}^{(22) M}\left(\left(k^{2} / L\right)^{p}\right)^{2} p^{2} .
\end{aligned}
$$

In this case, it is relatively easy to obtain that the coefficients are given by

$$
n_{g}^{(0) M}=8 p^{4}\left(\sqrt{m^{2}-k^{2}}\left(\left(k^{2} / L\right)^{p}\right)^{2}+\sqrt{m^{2}-k^{2}}\left(\left(k^{2} / L\right)^{p}\right)^{2} J(0, a k)^{2}\right.
$$




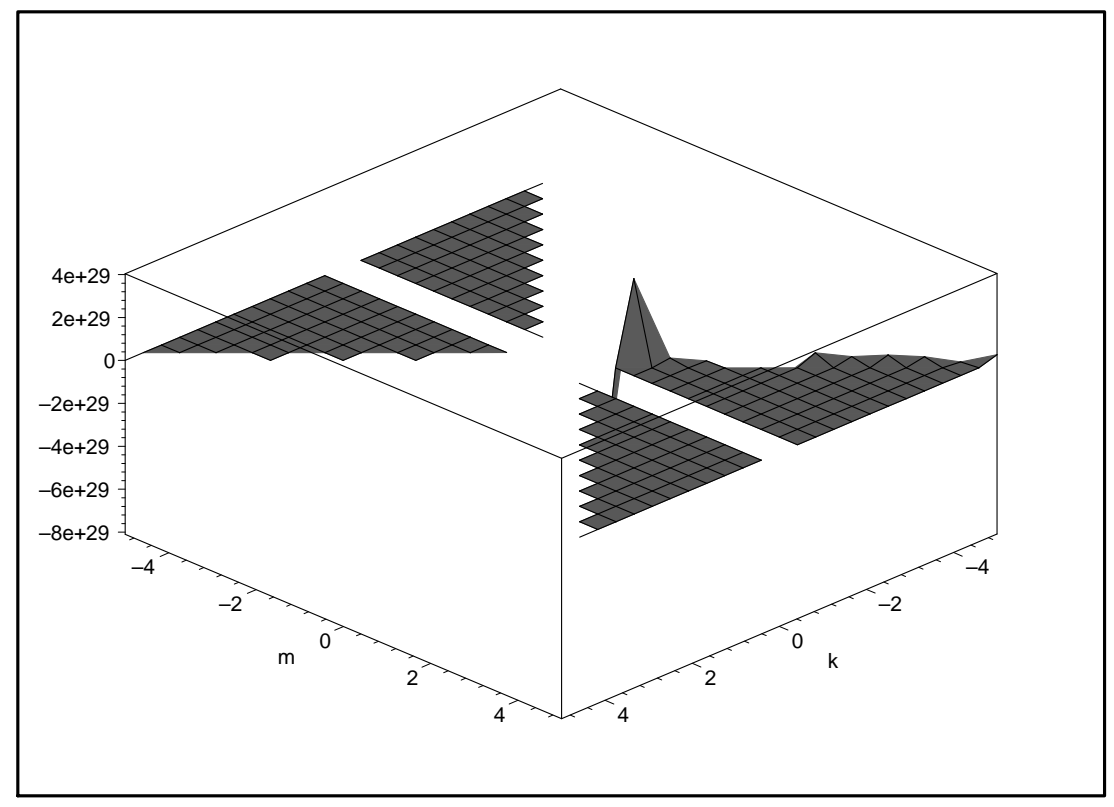

Figure 12: The determinant of the metric tensor plotted as a function of the transverse momentum and mass $k, m$, describing the fluctuations in massive rotating quarkonia.

$$
\begin{aligned}
& -2 m f\left(\left(k^{2} / L\right)^{p}\right)^{2} J(0, a k)+m f\left(\left(k^{2} / L\right)^{p}\right)^{2}+m f\left(\left(k^{2} / L\right)^{p}\right)^{2} J(0, a k)^{2} \\
& \left.-2 \sqrt{m^{2}-k^{2}}\left(\left(k^{2} / L\right)^{p}\right)^{2} J(0, a k)\right), \\
& n_{g}^{(12) M}=m f-2 m p f+2 m f J(1, a k) a k J(0, a k)+m f J(0, a k)^{2} \\
& +4 m p f J(0, a k)-2 m p f J(0, a k)^{2}-2 m f J(0, a k) \\
& +4 \sqrt{m^{2}-k^{2}} J(0, a k)^{2}+4 \sqrt{m^{2}-k^{2}} \\
& +4 \sqrt{m^{2}-k^{2}} J(0, a k) J(1, a k) a k-2 m f J(1, a k) a k \\
& -4 \sqrt{m^{2}-k^{2}} J(1, a k) a k-8 \sqrt{m^{2}-k^{2}} J(0, a k), \\
& n_{g}^{(13) M}=m f+2 m f J(1, a k) a k J(0, a k)-8 \sqrt{m^{2}-k^{2}} J(0, a k) \\
& +4 \sqrt{m^{2}-k^{2}}+4 \sqrt{m^{2}-k^{2}} J(0, a k) J(1, a k) a k \\
& +4 \sqrt{m^{2}-k^{2}} J(0, a k)^{2}-4 \sqrt{m^{2}-k^{2}} J(1, a k) a k \\
& -2 m f J(0, a k)-2 m f J(1, a k) a k+m f J(0, a k)^{2}, \\
& n_{g}^{(20) M}=m\left(f a^{2} k^{2} J(0, a k)-f a k J(1, a k)+f a k J(1, a k) J(0, a k)\right. \\
& \left.-f a^{2} k^{2} J(0, a k)^{2}\right)+2 \sqrt{m^{2}-k^{2}}\left(1+J(0, a k)^{2}\right. \\
& \left.+J(1, a k)^{2} a^{2} k^{2}+2 k J(0, a k) J(1, a k) a-2 k J(1, a k) a-2 J(0, a k)\right), \\
& n_{g}^{(21) M}=m\left(f a^{2} k^{2} J(0, a k)-f a^{2} k^{2} J(0, a k)^{2}+f a k J(1, a k) J(0, a k)\right. \\
& -f a k J(1, a k))+2 \sqrt{m^{2}-k^{2}}\left(1+J(1, a k)^{2} a^{2} k^{2}\right. \\
& \left.+J(0, a k)^{2}-2 k J(1, a k) a-2 J(0, a k)+2 k J(1, a k) a J(0, a k)\right), \\
& n_{g}^{(22) M}=m f\left(a k J(1, a k) J(0, a k)+a^{2} k^{2} J(0, a k)-a k J(1, a k)\right. \\
& \left.-a^{2} k^{2} J(0, a k)^{2}\right)+2 \sqrt{m^{2}-k^{2}}\left(1+J(1, a k)^{2} a^{2} k^{2}\right. \\
& \left.-2 J(0, a k)+2 k J(1, a k) a J(0, a k)+2 J(0, a k)^{2}-2 k J(1, a k) a\right) \text {. }
\end{aligned}
$$

As per the requirement of the positive definiteness of the determinant of the metric tensor, the global stability of underlying quarkonia with fluctuating $\{k, m\}$ leads to the following constraint

$$
n_{g}^{(0) M}+n_{g}^{(1) M} l(p)+n_{g}^{(2) M} l(p)^{2}<0 .
$$


The globally covariant Riemann curvature tensor $R_{k m k m}$ offers a similar property, as described in the foregoing subsection. Specifically, we find that the $R_{k m k m}$ has various factors of $\left\{\left(m^{2}-k^{2}\right)^{n / 2} \mid n \in Z\right\}$ and various powers of the logarithmic coupling $l(p)$. Some of the interesting terms of $R_{k m k m}$ are $-600 m^{6}\left(m^{2}-k^{2}\right)^{11 / 2} l(p)^{4} k^{18}$, $+712 m^{18}\left(m^{2}-k^{2}\right)^{9 / 2} l(p)^{4} k^{8}, \ldots$ and $-1244 m^{28}\left(m^{2}-k^{2}\right)^{7 / 2} l(p)^{4} k^{8}$. It is worth mentioning that $R_{k m k m}$ contains approximately 50,000 terms whose presentation is quite lengthy for the present paper. Nevertheless, the globally invariant scalar curvature and globally covariant Riemann curvature tensor can straightforwardly be obtained. As described in the previous subsection, the globally covariant physical properties of two parameter quarkonia follow directly from the product of two geometrically invariant quantities, viz. $R$ and $\|g\|$. As per our consideration of the foregoing subsections for the globally invariant scalar curvature $R$ and determinant of the metric tensor $\|g\|$, the covariant properties, viz., $R_{k m k m}$ follow directly for the two parameter quarkonia.

From Figs.(10) and (11), we see that the graphical views of the heat capacities are defined as pure components of the metric tensor. The plots have been depicted against the transverse momentum $k$ and mass $m$. In the present case, the choice of parameters is made as $L=22500, p=5 / 6, b=1$ and $a=100000$. Notice that the graphical nature of all thermodynamic interactions remains the same, as long as the parameter $b$ has a fixed sign.

The $k k$-heat capacity Fig. (10) shows a large variation of minima when going from a negative $k$ for a given positive mass $m \in(0,5)$. The order of instability turns out to be as high as $-10^{16}$. In this case, we further find in the other quarqants that the underlying quarkonia remains nearly stable in three distinct pathes.

On the other hand, the $\mathrm{mm}$-heat capacity Fig.(11) indicates a similar behavior of the negative variation. In contrast to the foregoing case, in this case we find that the local interactions are present only in four disjoint regions, all of which exclude the origin. As expected, the strength of the above interactions depends on the domain chosen in the $(k, m)$ plane, and thus the nature of the stability of quarkonia with the fluctuating $\{k, m\}$.

The corresponding plot for the determinant of the metric tensor depicted in the Fig.(12), shows the graphical nature of the determinant of the metric tensor. These plots explicate the of regions of the thermodynamic (un)stability for the underlying massive quarkonia lying in a given QCD phase. Specifically, the significance of the fluctuations of $\{k, m\}$ is that they show thermodynamical interactions in the strongly coupled massive quarkonia. The above figures indicate that the quarkonia can be highly unstable even in the linear Regge regime. As the non-linear effects become stronger and stronger, it is thus expected that the thermodynamic instability and correlations would grow further. This motivates us to extend our analysis to the general quarkonium configuration.

\subsection{Generic Quarkonia}

In the present subsection, we analyze the properties of general massive rotating quarkonia, when all parameters of the theory are allowed to fluctuate. To do so, let us consider the scale $q$, index of effective potential $p$ and angular momentum $J$ as the parameters of the present interest. In the framework of the Bloch-Nordsieck resummation, the strong QCD coupling takes the following form

$$
A(q, p, J)=\frac{1}{b} \frac{p}{\ln \left(1+p(q / L)^{p}\right)} \ln \left(\frac{\sqrt{J}+\sqrt{J-q}}{\sqrt{J}+\sqrt{J-q}}\right)(1-J(0, a \sqrt{q})) .
$$

After some simplification, we find that the components of the metric tensor are

$$
\begin{aligned}
g_{q q} & =-\frac{p}{4 b l(p)^{3} \exp (2 l(p)) q^{5 / 2}(J-q)^{3 / 2}}\left(n_{11}^{(0) G}+n_{11}^{(1) G} l(p)+n_{11}^{(2) G} l(p)^{2}\right), \\
g_{q p} & =\frac{1}{2 b l(p)^{3} \exp (2 l(p)) q^{3 / 2}(J-q)^{1 / 2}}\left(n_{12}^{(0) G}+n_{12}^{(1) G} l(p)+n_{12}^{(2) G} l(p)^{2}\right), \\
g_{q J} & =\frac{p}{2 b l(p)^{2} \exp (l(p)) q^{3 / 2}(J-q)^{3 / 2} J^{1 / 2}}\left(n_{13}^{(0) G}+n_{13}^{(1) G} l(p)\right), \\
g_{p p} & =\frac{f}{b l(p)^{3} \exp (2 l(p))}(-1+J(0, a \sqrt{q}))\left(n_{22}^{(0) G}+n_{22}^{(1) G} l(p)\right), \\
g_{p J} & =-\frac{1}{b l(p)^{2} \exp (l(p))(J-q)^{1 / 2} J^{1 / 2}}(-1+J(0, a \sqrt{q}))\left(n_{23}^{(0) G}+\left(l(p) n_{23}^{(1) G}\right),\right. \\
g_{J J} & =\frac{p}{2 b l(p)(J-q)^{3 / 2} J^{3 / 2}}(-1+J(0, a \sqrt{(q)}))(2 J-q),
\end{aligned}
$$


where the coefficients $\left\{n_{11}^{(1) G}, n_{11}^{(2) G}, n_{12}^{(1) G}, n_{12}^{(2) G}\right\}$ appearing in the components of the metric tensor factorize as mentioned before. In the powers of $p$, the exact factorizations are given as follows: (i) the $q q$-component

$$
\begin{aligned}
& n_{11}^{(1) G}=4 p^{2}(q / L)^{p} n_{11}^{(12) G}+4 p^{3}\left((q / L)^{p}\right)^{2} n_{11}^{(12) G} \\
& n_{11}^{(2) G}=n_{11}^{(20) G}+2 p(q / L)^{p} n_{11}^{(21) G}+p^{2}\left((q / L)^{p}\right)^{2}
\end{aligned}
$$

(ii) the $q p$-component

$$
\begin{aligned}
& n_{12}^{(1) G}=p(q / L)^{p} n_{12}^{(11) G}+p^{2}\left((q / L)^{p}\right)^{2} n_{12}^{(12) G}, \\
& n_{12}^{(2) G}=n_{12}^{(20) G}+2 p(q / L)^{p} n_{11}^{(21) G}+p^{2}\left((q / L)^{p}\right)^{2} n_{12}^{(22) G} .
\end{aligned}
$$

Without any approximation, we obtain that the factors in the numerator of the $q q$-components are given by

$$
\begin{aligned}
& n_{11}^{(0) G}=8 p^{4} f\left((q / L)^{p}\right)^{2} \sqrt{J-q}\left(q^{3 / 2}+\sqrt{q} J(0, a \sqrt{q}) J-\sqrt{q} J\right. \\
& \left.-q^{3 / 2} J(0, a \sqrt{q})\right) \text {, } \\
& n_{11}^{(12) G}=f \sqrt{J-q} q^{3 / 2}-f \sqrt{J-q} q^{3 / 2} J(0, a \sqrt{q})-f \sqrt{J-q} \sqrt{q} J \\
& +f J(1, a \sqrt{q}) a q \sqrt{J-q} J-f J(1, a \sqrt{q}) a q^{2} \sqrt{J-q} \\
& +f \sqrt{J-q} \sqrt{q} J(0, a \sqrt{q}) J-2 \sqrt{J} q^{3 / 2} J(0, a \sqrt{q}) \\
& -2 J^{3 / 2} \sqrt{q}+2 J^{3 / 2} \sqrt{q} J(0, a \sqrt{q})+2 \sqrt{J} q^{3 / 2} \\
& +p f \sqrt{J-q} \sqrt{q} J-p f \sqrt{J-q} \sqrt{q} J(0, a \sqrt{q}) J \\
& -p f \sqrt{J-q} q^{3 / 2}+p f \sqrt{J-q} q^{3 / 2} J(0, a \sqrt{q}), \\
& n_{11}^{(13) G}=f J(1, a \sqrt{q}) a q \sqrt{J-q} J-f J(1, a \sqrt{q}) a q^{2} \sqrt{J-q} \\
& -f \sqrt{J-q} \sqrt{q} J-f \sqrt{J-q} q^{3 / 2} J(0, a \sqrt{q}) \\
& +f \sqrt{J-q} \sqrt{q} J(0, a \sqrt{q}) J+f \sqrt{J-q} q^{3 / 2} \\
& -2 \sqrt{J} q^{3 / 2} J(0, a \sqrt{q})+2 J^{3 / 2} \sqrt{q} J(0, a \sqrt{q}) \\
& +2 \sqrt{J} q^{3 / 2}-2 J^{3 / 2} \sqrt{q}, \\
& n_{11}^{(20) G}=f a\left(a q^{5 / 2} \sqrt{J-q} J(0, a \sqrt{q})-a q^{3 / 2} \sqrt{J-q} J(0, a \sqrt{q}) J\right. \\
& \left.+2 q \sqrt{J-q} J(1, a \sqrt{q}) J-2 q^{2} \sqrt{J-q} J(1, a \sqrt{q})\right) \\
& +2 \sqrt{J}\left(2 J \sqrt{q} J(0, a \sqrt{q})-2 J(1, a \sqrt{q}) a q^{2}\right. \\
& \left.+2 J J(1, a \sqrt{q}) a q+3 q^{3 / 2}-4 J^{3 / 2} \sqrt{q}-3 q^{3 / 2} J(0, a \sqrt{q})\right), \\
& n_{11}^{(21) G}=f a^{2} q^{5 / 2} \sqrt{J-q} J(0, a \sqrt{q})-f a^{2} q^{3 / 2} \sqrt{J-q} J(0, a \sqrt{q}) J \\
& +2 f a q \sqrt{J-q} J(1, a \sqrt{q}) J-2 f a q^{2} \sqrt{J-q} J(1, a \sqrt{q}) \\
& -4 J^{3 / 2} \sqrt{q}+4 J^{3 / 2} J(1, a \sqrt{q}) a q-4 \sqrt{J} J(1, a \sqrt{q}) a q^{2} \\
& +4 J^{3 / 2} \sqrt{q} J(0, a \sqrt{q})+6 \sqrt{J} q^{3 / 2}-6 \sqrt{J} q^{3 / 2} J(0, a \sqrt{q}), \\
& n_{11}^{(22) G}=f a^{2} q^{5 / 2} \sqrt{J-q} J(0, a \sqrt{q})-f a^{2} q^{3 / 2} \sqrt{J-q} J(0, a \sqrt{q}) J \\
& +2 f J(1, a \sqrt{q}) a q \sqrt{J-q} J-2 f J(1, a \sqrt{q}) a q^{2} \sqrt{J-q} \\
& -4 \sqrt{J} J(1, a \sqrt{q}) a q^{2}+4 J^{3 / 2} \sqrt{q} J(0, a \sqrt{q}) \\
& -4 J^{3 / 2} \sqrt{q}+4 J^{3 / 2} J(1, a \sqrt{q}) a q+6 \sqrt{J} q^{3 / 2} \\
& -6 \sqrt{J} q^{3 / 2} J(0, a \sqrt{q}) \text {. }
\end{aligned}
$$

In a parallel way, the factors in the numerator of the $q p$-components turn out to be

$$
n_{12}^{(0) G}=4 p^{3} f\left((q / L)^{p}\right)^{2}(\sqrt{J-q} \sqrt{q}-\sqrt{J-q} \sqrt{q} J(0, a \sqrt{q})
$$




$$
\begin{aligned}
& +p \sqrt{J-q} \sqrt{q} \ln (q / L)-p \sqrt{J-q} \sqrt{q} J(0, a \sqrt{q}) \ln (q / L)), \\
n_{12}^{(11) G}= & 2 \sqrt{J} \sqrt{q}-2 p^{2} f \sqrt{J-q} \sqrt{q} \ln (q / L)-6 p f \sqrt{J-q} \sqrt{q} \\
& +2 p^{2} f \sqrt{J-q} \sqrt{q} J(0, a \sqrt{q}) \ln (q / L)-2 \sqrt{J} \sqrt{q} J(0, a \sqrt{q}) \\
& -f J(1, a \sqrt{q}) a q \sqrt{J-q}+2 p \sqrt{J} \sqrt{q} \ln (q / L) \\
& -2 p \sqrt{J} \sqrt{q} J(0, a \sqrt{q}) \ln (q / L)+6 p f \sqrt{J-q} \sqrt{q} J(0, a \sqrt{q}) \\
& -p f J(1, a \sqrt{q}) a q \sqrt{J-q} \ln (q / L), \\
n_{12}^{(12) G}= & 2 \sqrt{J} \sqrt{q}-f J(1, a \sqrt{q}) a q \sqrt{J-q}+2 p \sqrt{J} \sqrt{q} \ln (q / L) \\
& -2 \sqrt{J} \sqrt{q} J(0, a \sqrt{q})-2 p \sqrt{J} \sqrt{q} J(0, a \sqrt{q}) \ln (q / L) \\
& +4 p f \sqrt{J-q} \sqrt{q} J(0, a \sqrt{q})-4 p f \sqrt{J-q} \sqrt{q} \\
& -p f J(1, a \sqrt{q}) a q \sqrt{J-q} \ln (q / L), \\
n_{12}^{(20) G}= & f a q \sqrt{J-q} J(1, a \sqrt{q})+2 \sqrt{J} \sqrt{q} J(0, a \sqrt{q})-2 \sqrt{J} \sqrt{q}, \\
n_{12}^{(20) G}= & a q \sqrt{J-q} J(1, a \sqrt{q})+2 \sqrt{J} \sqrt{q} J(0, a \sqrt{q})-2 \sqrt{J} \sqrt{q}, \\
n_{12}^{(22) G}= & 2 \sqrt{J} \sqrt{q} J(0, a \sqrt{q})-2 \sqrt{J} \sqrt{q}+f a q \sqrt{J-q} J(1, a \sqrt{q}) .
\end{aligned}
$$

The factors in the numerator of the $q J$-components are expressed as

$$
\begin{aligned}
n_{13}^{(0) G}= & 2 p^{2}(q / L)^{p}\left(q^{3 / 2}+\sqrt{q} J(0, a \sqrt{q}) J-q^{3 / 2} J(0, a \sqrt{q})-\sqrt{q} J\right), \\
n_{13}^{(1) G}= & q\left(q^{1 / 2}-q^{1 / 2} J(0, a \sqrt{q})+J(1, a \sqrt{q}) a J-J(1, a \sqrt{q}) a q\right) \\
& +p(q / L)^{p}\left(q^{3 / 2}-q^{3 / 2} J(0, a \sqrt{q})+J(1, a \sqrt{q}) a q J-J(1, a \sqrt{q}) a q^{2}\right) .
\end{aligned}
$$

The corresponding factors in the numerator of the $p p$-components are given by

$$
\begin{aligned}
n_{22}^{(0) G}= & -2 p(q / L)^{2 p}\left(1+2 p \ln (q / L)+p^{2} \ln (q / L)^{2}\right), \\
n_{22}^{(1) G}= & (q / L)^{p}\left(2+4 p \ln (q / L)+p^{2} \ln (q / L)^{2}\right) \\
& +p(q / L)^{2 p}(1+2 p \ln (q / L)) .
\end{aligned}
$$

Finally, the factors in the numerator of the $p J$-components are

$$
\begin{aligned}
n_{23}^{(0) G} & =-p(q / L)^{p}-p^{2} \ln (q / L)(q / L)^{p}, \\
n_{23}^{(1) G} & =1+p(q / L)^{p} .
\end{aligned}
$$

Herewith, we observe that the principle components of the metric tensor, signifying self pair correlations, remain positive definite functions in non-trivial intervals of the parameters. The local stability of the configuration requires the following three simultaneously constraints. For the same sign of $\{p, b$, the thermodynamic stability enforces: (i) the $q q$ - fluctuations satisfy

$$
n_{11}^{(0) G}+n_{11}^{(1) G} l(p)+n_{11}^{(2) G} l(p)^{2},
$$

(ii) the $J J$ - fluctuations remain within the limiting values of the Bessel function

$$
\begin{aligned}
J(0, a \sqrt{q}) & >1,2 J<q, \\
& <1,2 J>q,
\end{aligned}
$$

and (iii) the $p p$ - fluctuations satisfy

$$
\begin{aligned}
n_{22}^{(0) G}+n_{22}^{(1) G} l(p) & >0, \quad J(0, a \sqrt{q})>1, \\
& <0, \quad J(0, a \sqrt{q})<1
\end{aligned}
$$

for the same sign of $\{f, b\}$. Correspondingly, the three dimensional views of the heat capacities are shown in the following plots for the general rotating massive case. For the purpose of the present and subsequent diagrammatic views, we shall focus on the case of $p=5 / 6$ and do not take any approximation against the 


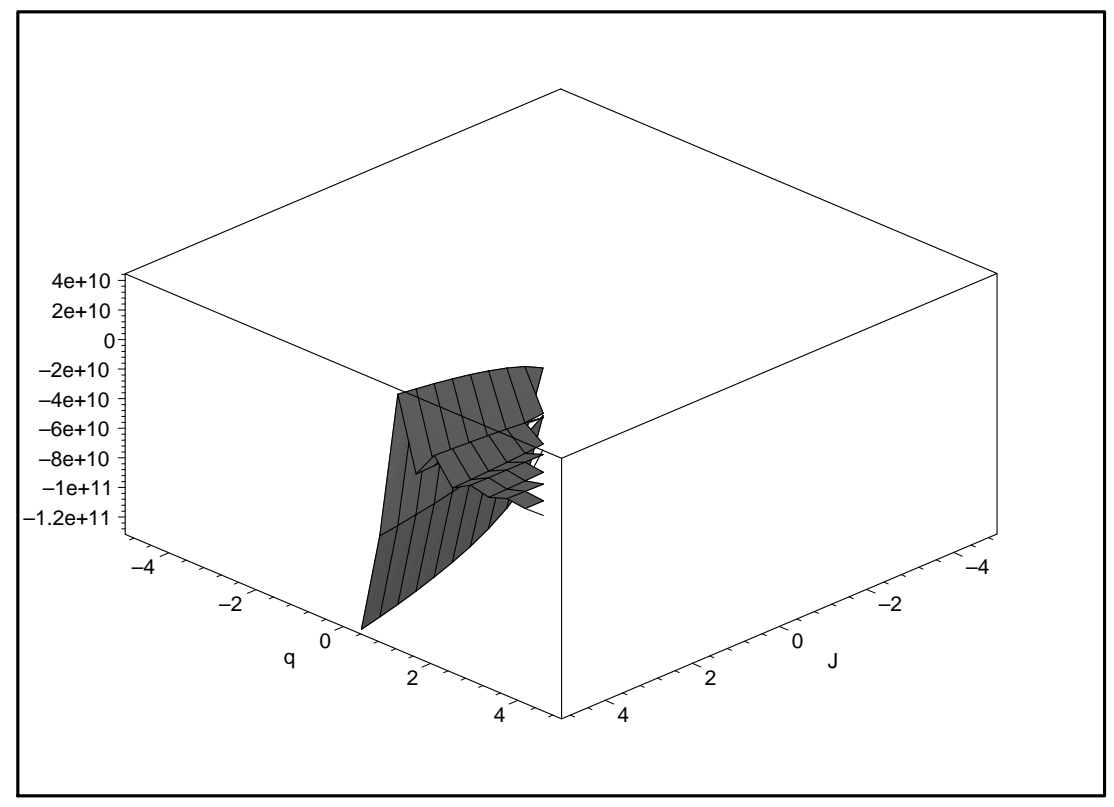

Figure 13: The $q q$-component of the metric tensor plotted as a function of $q$ and the angular momentum, $J$, describing the nature of the heat capacity of the $J J$-component for general massive rotating quarkonia.

Bessel function. Thereby, we graphically illustrate the local stability of the rotating quarkonia, which is in accordance with the above positivity constraints of the specific heat capacities.

As a function of scale parameter $q:=Q^{2}$ and angular momentum $J$, the $q q$-component is shown in the Fig.(13). As mentioned before, let us illustrate the present case for the same choice of the parameters, viz., $L=$ $22500, b=1$ and $a=100000$. Consequently, we find that the graphical nature of the underlying thermodynamic nature of the quarkonia remains the same, as long as the parameter $b$ has a fixed sign.

The $p p$-heat capacity is depicted in the Fig.(14). For a small $q$, this shows a large minimum of depth $10^{6}$, in the regime of the angular momentum $J \in(0,5)$. It turns out that the underlying $p p$-fluctuations of the quarkonia remain nearly stable in the other quadrants.

Similarly, the $J J$-heat capacity is shown in the corresponding Fig. (15), which indicates a similar behavior of the negative variation, as mentioned in the case of the $Q J$-plan. The silent feature of the $J J$-fluctuations is that we find the two distinct local behaviors for $J>0$ and $J<0$. In the limit of vanishing $q$ and $J$, the local interactions reach an order of $10^{4}$. For fluctuating $\{q, p, J\}$, the strength of the thermodynamic interactions depends on the domain chosen in the $(q, p, J)$ plane, and thus so does the thermodynamic stability of the quarkonia.

We further observe that the Gaussian fluctuations of the most general quarkonia comply with expected thermodynamic stability. Physically, the heat capacities under the fluctuations, which are defined as the selfpair correlations, remain positive quantities in the domain of the parameters and thus form the well-defined basis of the manifold $\left(M_{3}, g\right)$. Subsequently, our computation demonstrates the intrinsic geometric nature of the parametric fluctuations. The quarkonia remain globally stable under the fluctuations, if the associated principle minors, viz., $\left\{p_{2}, p_{3}\right\}$ remain positive definite functions. Herewith, the $q p$-surface is stable if we have a positive surface minor

$$
p_{S}^{G}:=-\frac{1}{4 b^{2} l(p)^{5} \exp (3 l(p)) q^{5 / 2}(J-q)^{3 / 2}}\left(n_{S}^{(0) G}+n_{g}^{(1) G} l(p)+n_{S}^{(2) G} l(p)^{2}+n_{S}^{(3) G} l(p)^{3}\right),
$$

where the $n_{S}^{(0) G}$ has the following illuminating factorization

$$
n_{S}^{(0) G}=8 f^{2}\left((q / L)^{p}\right)^{3}\left(p^{4} n_{S}^{(01) G}+p^{5} n_{S}^{(02) G}+p^{6} n_{S}^{(03) G}\right) .
$$

Interestingly, the factors $\left\{n_{S}^{(01) G}, n_{S}^{(02) G}, n_{S}^{(03) G}\right\}$ can be expressed as 


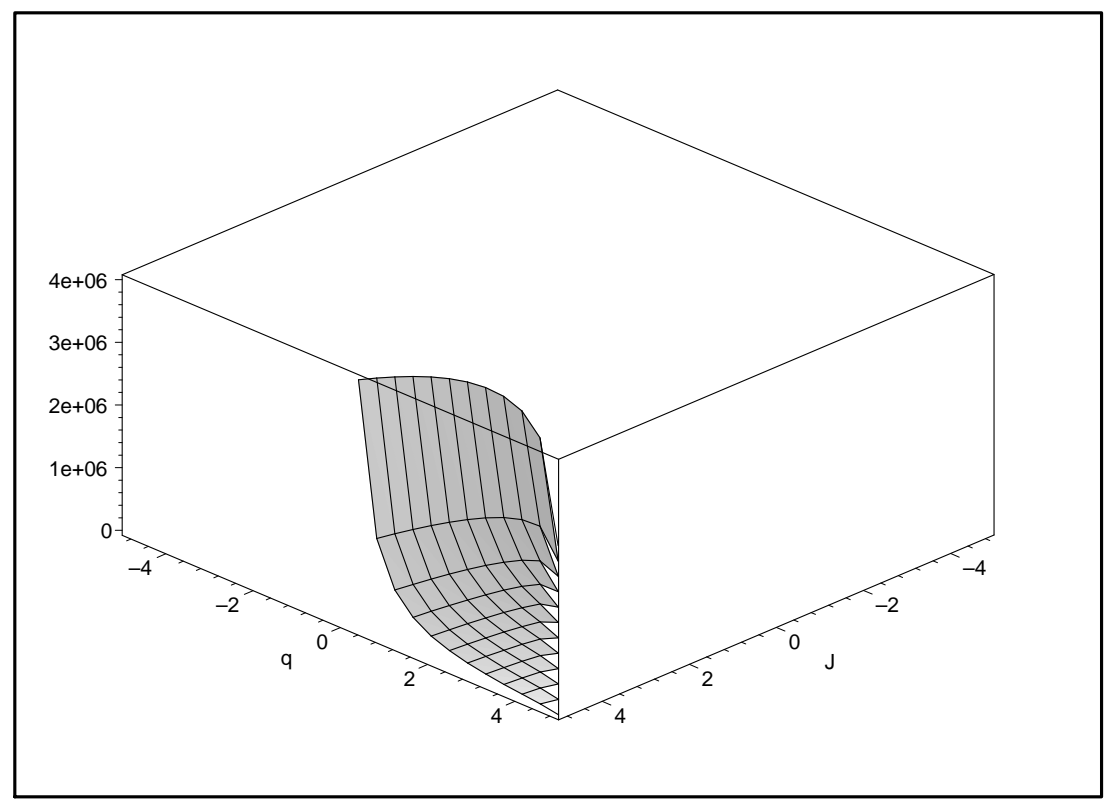

Figure 14: The $p p$-component of the metric tensor plotted as a function of the scale $q$, and the angular momentum, $J$, describing the nature of the heat capacity of the $J J$-component for general massive rotating quarkonia.

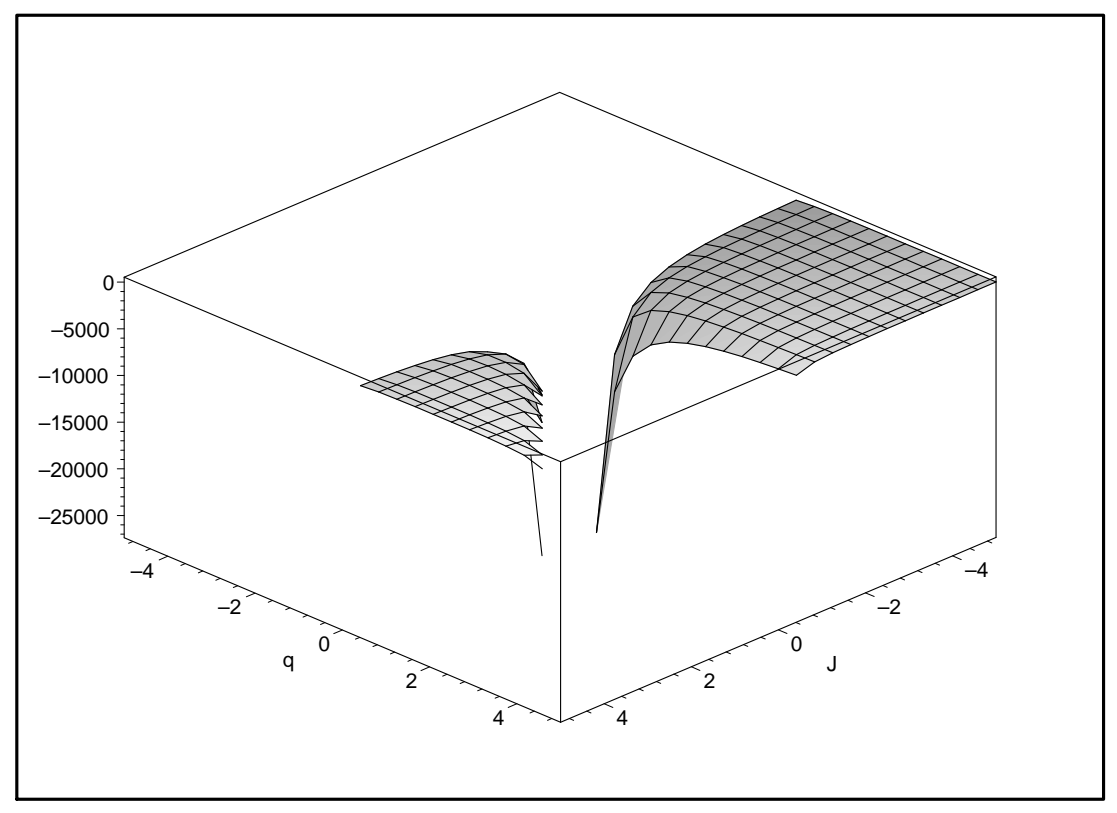

Figure 15: The $J J$-component of the metric tensor plotted as a function of scale and the angular momentum, viz., $q, J$, describing the nature of the heat capacity of the $J J$-component for general massive rotating quarkonia. 


$$
\begin{aligned}
n_{S}^{(01) G}= & q^{3 / 2} \sqrt{J-q}+q^{3 / 2} \sqrt{J-q} J(0, a \sqrt{q})^{2}-J \sqrt{q} \sqrt{J-q} \\
& -J \sqrt{q} \sqrt{J-q} J(0, a \sqrt{q})^{2}-2 q^{3 / 2} \sqrt{J-q} J(0, a \sqrt{q}) \\
& +2 J \sqrt{q} \sqrt{J-q} J(0, a \sqrt{q}) \\
n_{S}^{(02) G}= & 2 q^{3 / 2} \sqrt{J-q} \ln (q / L)-2 J \sqrt{q} \sqrt{J-q} J(0, a \sqrt{q})^{2} \ln (q / L) \\
& +2 q^{3 / 2} \sqrt{J-q} J(0, a \sqrt{q})^{2} \ln (q / L)+3 q^{3 / 2} \sqrt{J-q} \\
& -3 \sqrt{q} \sqrt{J-q} J J(0, a \sqrt{q})^{2}+3 q^{3 / 2} \sqrt{J-q} J(0, a \sqrt{q})^{2} \\
& +4 J \sqrt{q} \sqrt{J-q} J(0, a \sqrt{q}) \ln (q / L)-2 J \sqrt{q} \sqrt{J-q} \ln (q / L) \\
& -6 q^{3 / 2} \sqrt{J-q} J(0, a \sqrt{q})+6 \sqrt{q} \sqrt{J-q} J J(0, a \sqrt{q}) \\
& \left.-3 \sqrt{q} \sqrt{J-q} J-4 q^{3 / 2} \sqrt{J-q} J(0, a \sqrt{q}) \ln (q / L)\right), \\
& q^{3 / 2} \sqrt{J-q} \ln (q / L)^{2}+q^{3 / 2} \sqrt{J-q} J(0, a \sqrt{q})^{2} \ln (q / L)^{2} \\
& -J \sqrt{q} \sqrt{J-q} J(0, a \sqrt{q})^{2} \ln (q / L)^{2}-2 \sqrt{q} \sqrt{J-q} J \ln (q / L) \\
& +2 q^{3 / 2} \sqrt{J-q} J(0, a \sqrt{q})^{2} \ln (q / L)+2 J \sqrt{q} \sqrt{J-q} J(0, a \sqrt{q}) \ln (q / L)^{2} \\
& +2 q^{3 / 2} \sqrt{J-q} \ln (q / L)-2 q^{3 / 2} \sqrt{J-q} J(0, a \sqrt{q}) \ln (q / L)^{2} \\
& -2 \sqrt{q} \sqrt{J-q} J J(0, a \sqrt{q})^{2} \ln (q / L)+4 \sqrt{q} \sqrt{J-q} J J(0, a \sqrt{q}) \ln (q / L) \\
& -J \sqrt{q} \sqrt{J-q} \ln (q / L)^{2}-4 q^{3 / 2} \sqrt{J-q} J(0, a \sqrt{q}) \ln (q / L) .
\end{aligned}
$$

It is worth mentioning that the factors of the $l(p)$-terms can further be expressed as

$$
n_{S}^{(1) G}=(q 2 / L)^{2 p} n_{S}^{(12) G}+\left(\left(k^{2} / L\right)^{3 p} n_{S}^{(13) G}\right)
$$

with the following additional structures

$$
\begin{aligned}
& n_{S}^{(12) G}=n_{S}^{(120) G}+n_{S}^{(121) G} f+n_{S}^{(122) G} f^{2}, \\
& n_{S}^{(13) G}=n_{S}^{(130) G}+n_{S}^{(131) G} f+n_{S}^{(132) G} f^{2} .
\end{aligned}
$$

We find in particular that the 120-factor reduces to the following explicit expression

$$
\begin{aligned}
n_{S}^{(120) G}= & 4\left(\sqrt{q} \sqrt{J-q} J p^{2}+\sqrt{q} \sqrt{J-q} p^{4} J J(0, a \sqrt{q})^{2} \ln (q / L)^{2}\right. \\
& -2 q^{2} \sqrt{J} p^{3} f J(1, a \sqrt{q}) a \ln (q / L)-2 \sqrt{q} \sqrt{J-q} J J(0, a \sqrt{q}) p^{2} \\
& +2 \sqrt{q} \sqrt{J-q} p^{3} J J(0, a \sqrt{q})^{2} \ln (q / L)-2 \sqrt{q} \sqrt{J-q} p^{4} J J(0, a \sqrt{q}) \ln (q / L)^{2} \\
& \left.-8 \sqrt{q} \sqrt{J-q} p^{3} J J(0, a \sqrt{q}) \ln (q / L)\right) .
\end{aligned}
$$

The 121- and 122-factors factorize as follows

$$
\begin{aligned}
& n_{S}^{(121) G}=p^{2} n_{S}^{(1212) G}+2 p^{3} n_{S}^{(2113) G}+p^{4} n_{S}^{(1214) G} \\
& n_{S}^{(122) G}=p^{2} n_{S}^{(2122) G}+2 p^{3} n_{S}^{(2123) G}+p^{4} n_{S}^{(2124) G}+4 p^{5} n_{S}^{(2125) G}
\end{aligned}
$$

with the following expressions for the sub-factors

$$
\begin{aligned}
n_{S}^{(1212) G}= & \sqrt{J}\left(J q a J(1, a \sqrt{q})-q^{2} J(1, a \sqrt{q}) a+q^{2} J(1, a \sqrt{q}) a J(0, a \sqrt{q})\right. \\
& -J q a J(1, a \sqrt{q}) J(0, a \sqrt{q})-2 J \sqrt{q}-2 J \sqrt{q} J(0, a \sqrt{q})^{2}+3 q^{3 / 2} \\
& \left.+3 q^{3 / 2} J(0, a \sqrt{q})^{2}+4 J \sqrt{q} J(0, a \sqrt{q})-6 q^{3 / 2} J(0, a \sqrt{q})\right), \\
n_{S}^{(2113) G=} & \sqrt{J}\left(q^{2} J(0, a \sqrt{q}) \ln (q / L) J(1, a \sqrt{q}) a+J q J(1, a \sqrt{q}) a \ln (q / L)\right.
\end{aligned}
$$




$$
\begin{aligned}
& -J q J(1, a \sqrt{q}) a J(0, a \sqrt{q}) \ln (q / L)-2 J \sqrt{q} \ln (q / L)+3 q^{3 / 2} \\
& -2 J \sqrt{q} J(0, a \sqrt{q})^{2} \ln (q / L)-3 J \sqrt{q}+3 q^{3 / 2} J(0, a \sqrt{q})^{2} \ln (q / L) \\
& +3 q^{3 / 2} J(0, a \sqrt{q})^{2}-3 J \sqrt{q} J(0, a \sqrt{q})^{2}+3 q^{3 / 2} \ln (q / L) \\
& +4 J \sqrt{q} J(0, a \sqrt{q}) \ln (q / L)+6 J \sqrt{q} J(0, a \sqrt{q}) \\
& \left.-6 q^{3 / 2} J(0, a \sqrt{q}) \ln (q / L)-6 q^{3 / 2} J(0, a \sqrt{q})\right), \\
& n_{S}^{(1214) G}=\sqrt{J}\left(q^{2} J(1, a \sqrt{q}) a J(0, a \sqrt{q}) \ln (q / L)^{2}+J q \ln (q / L)^{2} J(1, a \sqrt{q}) a\right. \\
& -q^{2} \ln (q / L)^{2} J(1, a \sqrt{q}) a-J q J(0, a \sqrt{q}) \ln (q / L)^{2} J(1, a \sqrt{q}) a \\
& -2 J \sqrt{q} J(0, a \sqrt{q})^{2} \ln (q / L)^{2}+3 q^{3 / 2} J(0, a \sqrt{q})^{2} \ln (q / L)^{2} \\
& +3 q^{3 / 2} \ln (q / L)^{2}+4 J \sqrt{q} J(0, a \sqrt{q}) \ln (q / L)^{2} \\
& +4 q^{3 / 2} J(0, a \sqrt{q})^{2} \ln (q / L)+4 q^{3 / 2} \ln (q / L)-4 J \sqrt{q} \ln (q / L) \\
& -4 J \sqrt{q} J(0, a \sqrt{q})^{2} \ln (q / L)-6 q^{3 / 2} J(0, a \sqrt{q}) \ln (q / L)^{2} \\
& \left.+8 J \sqrt{q} J(0, a \sqrt{q}) \ln (q / L)-8 q^{3 / 2} J(0, a \sqrt{q}) \ln (q / L)\right) \text {. }
\end{aligned}
$$

The 122-factors take five powers of $p$, whose precise expressions take the form

$$
\begin{aligned}
& n_{S}^{(2122) G}=a q \sqrt{J-q}\left(q^{1 / 2} J a J(1, a \sqrt{q})^{2}-q^{3 / 2} a J(1, a \sqrt{q})^{2}+2 J q^{1 / 2} a J(0, a \sqrt{q})^{2}\right.
\end{aligned}
$$

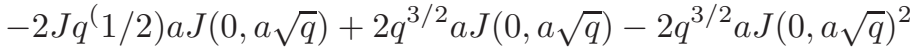

$$
\begin{aligned}
& -4 J J(1, a \sqrt{q}) J(0, a \sqrt{q})+4 q J(1, a \sqrt{q}) J(0, a \sqrt{q})-4 q J(1, a \sqrt{q}) \\
& +4 J J(1, a \sqrt{q})) \text {, } \\
& n_{S}^{(2123) G}=\sqrt{J-q}\left(q^{3 / 2} J a^{2} J(1, a \sqrt{q})^{2} \ln (q / L)-q^{5 / 2} J(1, a \sqrt{q})^{2} a^{2} \ln (q / L)\right. \\
& +2 J q^{3 / 2} a^{2} J(0, a \sqrt{q})^{2} \ln (q / L)+2 q^{5 / 2} a^{2} J(0, a \sqrt{q}) \ln (q / L) \\
& -2 q^{5 / 2} a^{2} J(0, a \sqrt{q})^{2} \ln (q / L)-2 J q^{3 / 2} a^{2} J(0, a \sqrt{q}) \ln (q / L) \\
& +4 q^{2} a J(1, a \sqrt{q}) J(0, a \sqrt{q}) \ln (q / L)-4 J q a J(1, a \sqrt{q}) J(0, a \sqrt{q}) \ln (q / L) \\
& +4 J q a J(1, a \sqrt{q}) \ln (q / L)-4 q^{3 / 2}+4 \sqrt{q} J p^{5} J(0, a \sqrt{q})^{2} \ln (q / L) \\
& -4 q^{2} a J(1, a \sqrt{q}) \ln (q / L)+6 q J a J(1, a \sqrt{q})-6 q J a J(1, a \sqrt{q}) J(0, a \sqrt{q}) \\
& +6 q^{2} a J(1, a \sqrt{q}) J(0, a \sqrt{q})-6 q^{2} a J(1, a \sqrt{q})+8 q^{3 / 2} J(0, a \sqrt{q}) \\
& -8 J \sqrt{q} J(0, a \sqrt{q})) \\
& n_{S}^{(2124) G}=\sqrt{J-q}\left(q^{3 / 2} J J(1, a \sqrt{q})^{2} a^{2} \ln (q / L)^{2}-q^{5 / 2} J(1, a \sqrt{q})^{2} a^{2} \ln (q / L)^{2}\right. \\
& +2 q^{5 / 2} a^{2} J(0, a \sqrt{q}) \ln (q / L)^{2}-2 q^{5 / 2} a^{2} J(0, a \sqrt{q})^{2} \ln (q / L)^{2} \\
& -2 J q^{3 / 2} a^{2} J(0, a \sqrt{q}) \ln (q / L)^{2}+2 J q^{3 / 2} a^{2} J(0, a \sqrt{q})^{2} \ln (q / L)^{2} \\
& -4 J q a J(1, a \sqrt{q}) J(0, a \sqrt{q}) \ln (q / L)^{2}+4 J q a J(1, a \sqrt{q}) \ln (q / L)^{2} \\
& +4 q^{2} a J(1, a \sqrt{q}) J(0, a \sqrt{q}) \ln (q / L)^{2}-4 q^{2} a J(1, a \sqrt{q}) \ln (q / L)^{2} \\
& +8 q^{2} J(0, a \sqrt{q}) \ln (q / L) a J(1, a \sqrt{q})-8 q J J(0, a \sqrt{q}) \ln (q / L) a J(1, a \sqrt{q}) \\
& +8 q J \ln (q / L) a J(1, a \sqrt{q})-8 q^{2} J(1, a \sqrt{q}) a \ln (q / L) \\
& +16 J \sqrt{q} J(0, a \sqrt{q})^{2} \ln (q / L)+16 J \sqrt{q} \ln (q / L) \\
& -16 q^{3 / 2} J(0, a \sqrt{q})^{2} \ln (q / L)-16 q^{3 / 2} \ln (q / L) \\
& +28 \sqrt{q} J J(0, a \sqrt{q})^{2}-28 q^{3 / 2} J(0, a \sqrt{q})^{2} \\
& +28 \sqrt{q} J-28 q^{3 / 2}-32 J \sqrt{q} J(0, a \sqrt{q}) \ln (q / L) \\
& \left.+32 q^{3 / 2} J(0, a \sqrt{q}) \ln (q / L)-56 \sqrt{q} J J(0, a \sqrt{q})+56 q^{3 / 2} J(0, a \sqrt{q})\right), \\
& n_{S}^{(2125) G}=\sqrt{J-q}\left(J \sqrt{q} \ln (q / L)^{2}+J \sqrt{q} J(0, a \sqrt{q})^{2} \ln (q / L)^{2}-q^{3 / 2} \ln (q / L)^{2}\right. \\
& -2 q^{3 / 2} J(0, a \sqrt{q})^{2} \ln (q / L)-2 J \sqrt{q} J(0, a \sqrt{q}) \ln (q / L)^{2}+2 \sqrt{q} J \ln (q / L) \\
& +2 q^{3 / 2} J(0, a \sqrt{q}) \ln (q / L)^{2}-4 \sqrt{q} J J(0, a \sqrt{q}) \ln (q / L) \\
& \left.+4 q^{3 / 2} J(0, a \sqrt{q}) \ln (q / L)\right) \text {. }
\end{aligned}
$$


Further, we take advantage of the following factorizations for the 131 and 132 expressions

$$
\begin{aligned}
& n_{S}^{(131) G}=4 p^{3} n_{S}^{(1313) G}+8 p^{4} n_{S}^{(1314) G}+4 p^{5} n_{S}^{(1315) G}, \\
& n_{S}^{(132) G}=p^{3} n_{S}^{(1323) G}+2 p^{4} n_{S}^{(1324) G}+p^{5} n_{S}^{(1325) G} .
\end{aligned}
$$

Subsequently, the factors of 130 and 131 appear in the powers of the $p$, and are given by

$$
\begin{aligned}
& n_{S}^{(130) G}=4 \sqrt{q} \sqrt{J-q} p^{3} J\left(1+p^{2} J(0, a \sqrt{q})^{2} \ln (q / L)^{2}+J(0, a \sqrt{q})^{2}\right. \\
& +p^{2} \ln (q / L)^{2}+2 p \ln (q / L)+2 p J(0, a \sqrt{q})^{2} \ln (q / L)-2 J(0, a \sqrt{q}) \\
& \left.-2 p^{2} J(0, a \sqrt{q}) \ln (q / L)^{2}-4 p J(0, a \sqrt{q}) \ln (q / L)\right) \text {, } \\
& n_{S}^{(1313) G}=J^{3 / 2} q a J(1, a \sqrt{q})-q^{2} \sqrt{J} J(1, a \sqrt{q}) a+q^{2} a J(1, a \sqrt{q}) \sqrt{J} J(0, a \sqrt{q}) \\
& -J^{3 / 2} q J(1, a \sqrt{q}) a J(0, a \sqrt{q})-2 J^{3 / 2} \sqrt{q}-2 J^{3 / 2} \sqrt{q} J(0, a \sqrt{q})^{2} \\
& +3 q^{3 / 2} \sqrt{J}+3 q^{3 / 2} \sqrt{J} J(0, a \sqrt{q})^{2}+4 J^{3 / 2} \sqrt{q} J(0, a \sqrt{q}) \\
& -6 q^{3 / 2} \sqrt{J} J(0, a \sqrt{q}) \text {, } \\
& n_{S}^{(1314) G}=J^{3 / 2} q J(1, a \sqrt{q}) a \ln (q / L)+q^{2} \sqrt{J} J(1, a \sqrt{q}) a J(0, a \sqrt{q}) \ln (q / L) \\
& -J^{3 / 2} q J(1, a \sqrt{q}) a J(0, a \sqrt{q}) \ln (q / L)-q^{2} \sqrt{J} J(1, a \sqrt{q}) a \ln (q / L) \\
& -2 J^{3 / 2} \sqrt{q} J(0, a \sqrt{q})^{2} \ln (q / L)-2 J^{3 / 2} \sqrt{q} \ln (q / L)+3 q^{3 / 2} \sqrt{J} \ln (q / L) \\
& +3 q^{3 / 2} \sqrt{J}+3 q^{3 / 2} \sqrt{J} J(0, a \sqrt{q})^{2}-3 J^{3 / 2} \sqrt{q}-3 J^{3 / 2} \sqrt{q} J(0, a \sqrt{q})^{2} \\
& +3 q^{3 / 2} \sqrt{J} J(0, a \sqrt{q})^{2} \ln (q / L)+4 J^{3 / 2} \sqrt{q} J(0, a \sqrt{q}) \ln (q / L) \\
& +6 J^{3 / 2} \sqrt{q} J(0, a \sqrt{q})-6 q^{3 / 2} \sqrt{J} J(0, a \sqrt{q})-6 q^{3 / 2} \sqrt{J} J(0, a \sqrt{q}) \ln (q / L), \\
& n_{S}^{(1315) G}=J^{3 / 2} q \ln (q / L)^{2} J(1, a \sqrt{q}) a-q^{2} \sqrt{J} J(1, a \sqrt{q}) a \ln (q / L)^{2} \\
& +q^{2} \sqrt{J} J(0, a \sqrt{q}) \ln (q / L)^{2} J(1, a \sqrt{q}) a-J^{3 / 2} q J(0, a \sqrt{q}) \ln (q / L)^{2} J(1, a \sqrt{q}) a \\
& -2 J^{3 / 2} \sqrt{q} \ln (q / L)^{2}-2 J^{3 / 2} \sqrt{q} J(0, a \sqrt{q})^{2} \ln (q / L)^{2}+3 q^{3 / 2} \sqrt{J} \ln (q / L)^{2} \\
& +3 q^{3 / 2} \sqrt{J} J(0, a \sqrt{q})^{2} \ln (q / L)^{2}-4 J^{3 / 2} \sqrt{q} J(0, a \sqrt{q})^{2} \ln (q / L) \\
& -4 J^{3 / 2} \sqrt{q} \ln (q / L)+4 q^{3 / 2} \sqrt{J} J(0, a \sqrt{q})^{2} \ln (q / L)+4 q^{3 / 2} \sqrt{J} \ln (q / L) \\
& +4 J^{3 / 2} \sqrt{q} J(0, a \sqrt{q}) \ln (q / L)^{2}-6 q^{3 / 2} \sqrt{J} J(0, a \sqrt{q}) \ln (q / L)^{2} \\
& -8 q^{3 / 2} \sqrt{J} J(0, a \sqrt{q}) \ln (q / L)+8 J^{3 / 2} \sqrt{q} J(0, a \sqrt{q}) \ln (q / L) .
\end{aligned}
$$

The factors of 132-powers, appearing as the powers of the $p$, are

$$
\begin{aligned}
& n_{S}^{(1323) G}=q^{3 / 2} \sqrt{J-q} J J(1, a \sqrt{q})^{2} a^{2}-q^{5 / 2} \sqrt{J-q} a^{2} J(1, a \sqrt{q})^{2}
\end{aligned}
$$

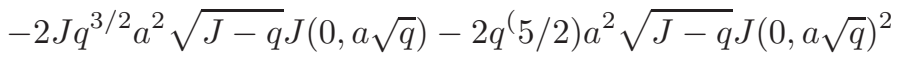

$$
\begin{aligned}
& +2 q^{5 / 2} a^{2} \sqrt{J-q} J(0, a \sqrt{q})-4 J q J(1, a \sqrt{q}) a \sqrt{J-q} J(0, a \sqrt{q}) \\
& +4 J q a \sqrt{J-q} J(1, a \sqrt{q})+2 J q^{3 / 2} a^{2} \sqrt{J-q} J(0, a \sqrt{q})^{2} \\
& +4 q^{2} a \sqrt{J-q} J(1, a \sqrt{q}) J(0, a \sqrt{q})-4 q^{2} a \sqrt{J-q} J(1, a \sqrt{q}), \\
& n_{S}^{(1324) G}=q^{3 / 2} \sqrt{J-q} J a^{2} J(1, a \sqrt{q})^{2} \ln (q / L)-q^{5 / 2} \sqrt{J-q} a^{2} J(1, a \sqrt{q})^{2} \ln (q / L) \\
& +2 J \sqrt{q} \sqrt{J-q}-2 q^{3 / 2} \sqrt{J-q}+2 J \sqrt{q} \sqrt{J-q} J(0, a \sqrt{q})^{2}
\end{aligned}
$$

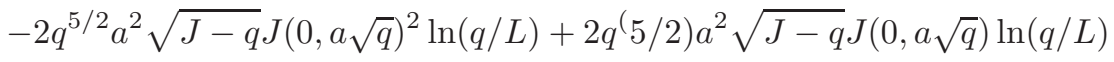

$$
\begin{aligned}
& -2 J q^{3 / 2} a^{2} \sqrt{J-q} J(0, a \sqrt{q}) \ln (q / L)+2 J q^{3 / 2} a^{2} \sqrt{J-q} J(0, a \sqrt{q})^{2} \ln (q / L) \\
& -2 q^{3 / 2} \sqrt{J-q} J(0, a \sqrt{q})^{2}-4 q^{2} J(1, a \sqrt{q}) a \sqrt{J-q} \ln (q / L) \\
& +4 J q J(1, a \sqrt{q}) a \sqrt{J-q} \ln (q / L)-4 J \sqrt{q} \sqrt{J-q} J(0, a \sqrt{q}) \\
& +4 q^{3 / 2} \sqrt{J-q} J(0, a \sqrt{q})-4 J q a \sqrt{J-q} J(1, a \sqrt{q}) J(0, a \sqrt{q}) \ln (q / L)
\end{aligned}
$$




$$
\begin{aligned}
+ & 4 q^{2} J(1, a \sqrt{q}) a \sqrt{J-q} J(0, a \sqrt{q}) \ln (q / L)-6 q^{2} \sqrt{J-q} J(1, a \sqrt{q}) a \\
& +6 q^{2} \sqrt{J-q} J(0, a \sqrt{q}) J(1, a \sqrt{q}) a+6 q \sqrt{J-q} J J(1, a \sqrt{q}) a \\
& -6 q \sqrt{J-q} J J(0, a \sqrt{q}) J(1, a \sqrt{q}) a, \\
n_{S}^{(1325) G}= & q^{3 / 2} \sqrt{J-q} J J(1, a \sqrt{q})^{2} a^{2} \ln (q / L)^{2}-q^{5 / 2} \sqrt{J-q} J(1, a \sqrt{q})^{2} a^{2} \ln (q / L)^{2} \\
+ & 2 q^{5 / 2} a^{2} \sqrt{J-q} J(0, a \sqrt{q}) \ln (q / L)^{2}+2 J q^{3 / 2} a^{2} \sqrt{J-q} J(0, a \sqrt{q})^{2} \ln (q / L)^{2} \\
& -2 q^{5 / 2} a^{2} \sqrt{J-q} J(0, a \sqrt{q})^{2} \ln (q / L)^{2}-2 J q^{3 / 2} a^{2} \sqrt{J-q} J(0, a \sqrt{q}) \ln (q / L)^{2} \\
& +4 q^{2} J(1, a \sqrt{q}) a \sqrt{J-q} J(0, a \sqrt{q}) \ln (q / L)^{2}+4 J q a \sqrt{J-q} J(1, a \sqrt{q}) \ln (q / L)^{2} \\
& -4 J q J(1, a \sqrt{q}) a \sqrt{J-q} J(0, a \sqrt{q}) \ln (q / L)^{2}-4 q^{2} J(1, a \sqrt{q}) a \sqrt{J-q} \ln (q / L)^{2} \\
& -8 q^{3 / 2} \sqrt{J-q} \ln (q / L)-8 q^{3 / 2} \sqrt{J-q} J(0, a \sqrt{q})^{2} \ln (q / L)+8 J \sqrt{q} \sqrt{J-q} \ln (q / L) \\
+ & 8 J \sqrt{q} \sqrt{J-q} J(0, a \sqrt{q})^{2} \ln (q / L)+8 q \sqrt{J-q} J J(1, a \sqrt{q}) a \ln (q / L) \\
& -8 q \sqrt{J-q} J(0, a \sqrt{q}) \ln (q / L) J(1, a \sqrt{q}) a-8 q^{2} \sqrt{J-q} J(1, a \sqrt{q}) a \ln (q / L) \\
+ & 8 q^{2} \sqrt{J-q} J(0, a \sqrt{q}) \ln (q / L) J(1, a \sqrt{q}) a+16 \sqrt{q} \sqrt{J-q} J \\
+ & 16 \sqrt{q} \sqrt{J-q} J J(0, a \sqrt{q})^{2}-16 J \sqrt{q} \sqrt{J-q} J(0, a \sqrt{q}) \ln (q / L) \\
& -16 q^{3 / 2} \sqrt{J-q}+16 q^{3 / 2} \sqrt{J-q} J(0, a \sqrt{q}) \ln (q / L)-16 q^{3 / 2} \sqrt{J-q} J(0, a \sqrt{q})^{2} \\
& -32 \sqrt{q} \sqrt{J-q} J J(0, a \sqrt{q})+32 q^{3 / 2} \sqrt{J-q} J(0, a \sqrt{q}) .
\end{aligned}
$$

It turns out that the factor of the $l(p)^{2}$ terms can be expressed as

$$
n_{S}^{(2) G}=(q / L)^{p} n_{S}^{(21) G}+(q / L)^{2 p} n_{S}^{(22) G}+\left((q / L)^{3 p} n_{S}^{(23) G}\right)
$$

with the following factorizations

$$
\begin{aligned}
& n_{S}^{(21) G}=n_{S}^{(210) G}+n_{S}^{(211) G} f+n_{S}^{(212) G} f^{2}, \\
& n_{S}^{(22) G}=n_{S}^{(220) G}+n_{S}^{(221) G} f+n_{S}^{(222) G} f^{2}, \\
& n_{S}^{(23) G}=n_{S}^{(230) G}+n_{S}^{(231) G} f+n_{S}^{(232) G} f^{2}
\end{aligned}
$$

and sub-factorizations of the following specific forms

$$
\begin{aligned}
& n_{S}^{(211) G}=4 p n_{S}^{(2111) G}+8 p^{2} n_{S}^{(2112) G}+2 p^{3} n_{S}^{(2113) G} \\
& n_{S}^{(212) G}=2 p n_{S}^{(2121) G}+2 p^{2} n_{S}^{(2122) G}+p^{3} n_{S}^{(2123) G} .
\end{aligned}
$$

The precise sub-factors of the 21-factorizations are

$$
\begin{aligned}
n_{S}^{(210) G}= & -8 \sqrt{q} \sqrt{J-q} J p\left(1+J(0, a \sqrt{q})^{2}+p \ln (q / L)-2 J(0, a \sqrt{q})\right. \\
& \left.+p J(0, a \sqrt{q})^{2} \ln (q / L)-2 p J(0, a \sqrt{q}) \ln (q / L)\right) \\
n_{S}^{(2111) G}= & 2 J^{3 / 2} \sqrt{q} J(0, a \sqrt{q})^{2}+2 J^{3 / 2} \sqrt{q}-2 q^{3 / 2} \sqrt{J} J(0, a \sqrt{q})^{2} \\
& -3 q^{3 / 2} \sqrt{J}-4 J^{3 / 2} \sqrt{q} J(0, a \sqrt{q})+6 q^{3 / 2} \sqrt{J} J(0, a \sqrt{q}) \\
n_{S}^{(2112) G}= & J^{3 / 2} q J(1, a \sqrt{q}) a J(0, a \sqrt{q}) \ln (q / L)+q^{2} \sqrt{J} J(1, a \sqrt{q}) a \ln (q / L) \\
& -J^{3 / 2} q J(1, a \sqrt{q}) a \ln (q / L)-q^{2} \sqrt{J} J(1, a \sqrt{q}) a J(0, a \sqrt{q}) \ln (q / L) \\
& +2 J^{3 / 2} \sqrt{q} J(0, a \sqrt{q})^{2} \ln (q / L)+2 J^{3 / 2} \sqrt{q} \ln (q / L) \\
& -3 q^{3 / 2} \sqrt{J} J(0, a \sqrt{q})^{2} \ln (q / L)-3 q^{3 / 2} \sqrt{J} J(0, a \sqrt{q})^{2} \\
& -3 q^{3 / 2} \sqrt{J}-3 q^{3 / 2} \sqrt{J} \ln (q / L)+3 J^{3 / 2} \sqrt{q} \\
& +3 J^{3 / 2} \sqrt{q} J(0, a \sqrt{q})^{2}-4 J^{3 / 2} \sqrt{q} J(0, a \sqrt{q}) \ln (q / L) \\
& -6 J^{3 / 2} \sqrt{q} J(0, a \sqrt{q})+6 q^{3 / 2} \sqrt{J} J(0, a \sqrt{q})
\end{aligned}
$$




$$
\begin{aligned}
& +6 q^{3 / 2} \sqrt{J} J(0, a \sqrt{q}) \ln (q / L), \\
n_{S}^{(2113) G=} & 2 J^{3 / 2} \sqrt{q} \ln (q / L)^{2}+2 q^{2} \sqrt{J} J(1, a \sqrt{q}) a \ln (q / L)^{2} \\
& -2 J^{3 / 2} q J(1, a \sqrt{q}) a \ln (q / L)^{2}-2 q^{2} \sqrt{J} J(1, a \sqrt{q}) a J(0, a \sqrt{q}) \ln (q / L)^{2} \\
& +2 J^{3 / 2} q J(1, a \sqrt{q}) a J(0, a \sqrt{q}) \ln (q / L)^{2}-3 q^{3 / 2} \sqrt{J} \ln (q / L)^{2} \\
& -3 q^{3 / 2} \sqrt{J} J(0, a \sqrt{q})^{2} \ln (q / L)^{2}+4 J^{3 / 2} \sqrt{q} \ln (q / L) \\
& -4 J^{3 / 2} \sqrt{q} J(0, a \sqrt{q}) \ln (q / L)^{2}-4 q^{3 / 2} \sqrt{J} \ln (q / L) \\
& -4 q^{3 / 2} \sqrt{J} J(0, a \sqrt{q})^{2} \ln (q / L)+4 J^{3 / 2} \sqrt{q} J(0, a \sqrt{q})^{2} \ln (q / L) \\
& +6 q^{3 / 2} \sqrt{J} J(0, a \sqrt{q}) \ln (q / L)^{2}+6 q^{3 / 2} \sqrt{J} J(0, a \sqrt{q}) \ln (q / L) \\
& +2 J^{3 / 2} \sqrt{q} J(0, a \sqrt{q})^{2} \ln (q / L)^{2}-8 J^{3 / 2} \sqrt{q} J(0, a \sqrt{q}) \ln (q / L) .
\end{aligned}
$$

For the 212-components, we have the following factors

$$
\begin{aligned}
& n_{S}^{(2121) G}=\sqrt{J-q}\left(q^{5 / 2} a^{2} J(1, a \sqrt{q})^{2}+J q^{3 / 2} a^{2} J(0, a \sqrt{q})+q^{(5 / 2) a^{2} J(0, a \sqrt{q})^{2}}\right.
\end{aligned}
$$

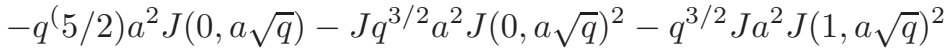

$$
\begin{aligned}
& +2 J q a J(1, a \sqrt{q}) J(0, a \sqrt{q})-2 q^{2} a J(1, a \sqrt{q}) J(0, a \sqrt{q})+2 q^{2} a J(1, a \sqrt{q}) \\
& -2 J q a J(1, a \sqrt{q})) \text {, } \\
& n_{S}^{(2122) G}=\sqrt{J-q}\left(q^{5 / 2} a^{2} J(1, a \sqrt{q})^{2} \ln (q / L)-q^{3 / 2} J a^{2} J(1, a \sqrt{q})^{2} \ln (q / L)\right. \\
& -2 q^{5 / 2} a^{2} J(0, a \sqrt{q}) \ln (q / L)-2 J q^{3 / 2} a^{2} J(0, a \sqrt{q})^{2} \ln (q / L) \\
& +2 q^{5 / 2} a^{2} J(0, a \sqrt{q})^{2} \ln (q / L)-4 q^{2} a J(1, a \sqrt{q}) J(0, a \sqrt{q}) \ln (q / L) \\
& +4 J q a J(1, a \sqrt{q}) J(0, a \sqrt{q}) \ln (q / L)+4 q J a J(1, a \sqrt{q}) J(0, a \sqrt{q}) \\
& +2 J q^{3 / 2} a^{2} J(0, a \sqrt{q}) \ln (q / L)-4 J q a J(1, a \sqrt{q}) \ln (q / L) \\
& +4 q^{2} a J(1, a \sqrt{q}) \ln (q / L)-6 q J a J(1, a \sqrt{q}) \\
& \left.-6 q^{2} a J(1, a \sqrt{q}) J(0, a \sqrt{q})+6 q^{2} a J(1, a \sqrt{q})\right), \\
& n_{S}^{(2123) G}=\sqrt{J-q}\left(q^{5 / 2} a^{2} J(0, a \sqrt{q})^{2} \ln (q / L)^{2}-q^{5 / 2} a^{2} J(0, a \sqrt{q}) \ln (q / L)^{2}\right. \\
& +J q^{3 / 2} a^{2} J(0, a \sqrt{q}) \ln (q / L)^{2}-J q^{3 / 2} a^{2} J(0, a \sqrt{q})^{2} \ln (q / L)^{2} \\
& -2 q^{2} a J(1, a \sqrt{q}) J(0, a \sqrt{q}) \ln (q / L)^{2}+2 J q a J(1, a \sqrt{q}) J(0, a \sqrt{q}) \ln (q / L)^{2} \\
& +2 q^{2} a J(1, a \sqrt{q}) \ln (q / L)^{2}-2 J q a J(1, a \sqrt{q}) \ln (q / L)^{2} \\
& +4 q^{2} a J(1, a \sqrt{q}) \ln (q / L)+4 q J a J(1, a \sqrt{q}) J(0, a \sqrt{q}) \ln (q / L) \\
& \left.-4 q J a J(1, a \sqrt{q}) \ln (q / L)-4 q^{2} a J(1, a \sqrt{q}) J(0, a \sqrt{q}) \ln (q / L)\right) \text {. }
\end{aligned}
$$

Similarly, it follows that the 22- factors obey the following particular relations

$$
\begin{aligned}
& n_{S}^{(221) G}=2 p^{2} n_{S}^{(2121) G}+4 p^{3} n_{S}^{(2122) G}+2 p^{4} n_{S}^{(2213) G}, \\
& n_{S}^{(222) G}=p^{2} n_{S}^{(2221) G}+2 p^{3} n_{S}^{(2222) G}+p^{4} n_{S}^{(2223) G} .
\end{aligned}
$$

In the above relations, the factors of the 220 and 221-components are given by

$$
\begin{aligned}
n_{S}^{(220) G}= & 16 J \sqrt{q} \sqrt{J-q} p^{2}(2 J(0, a \sqrt{q})+2 p J(0, a \sqrt{q}) \ln (q / L) \\
& \left.-p J(0, a \sqrt{q})^{2} \ln (q / L)-p \ln (q / L)-J(0, a \sqrt{q})^{2}-1\right), \\
n_{S}^{(2121) G}= & \sqrt{J}\left(2 J q J(1, a \sqrt{q}) a-2 q^{2} J(1, a \sqrt{q}) a+2 q^{2} J(1, a \sqrt{q}) a J(0, a \sqrt{q})\right. \\
& -2 J q J(1, a \sqrt{q}) a J(0, a \sqrt{q})+6 J \sqrt{q} J(0, a \sqrt{q})^{2}-9 q^{3 / 2} J(0, a \sqrt{q})^{2} \\
& \left.+6 J \sqrt{q}-9 q^{3 / 2}-12 J \sqrt{q} J(0, a \sqrt{q})+18 q^{3 / 2} J(0, a \sqrt{q})\right), \\
n_{S}^{(2122) G}= & \sqrt{J}\left(2 q^{2} J(1, a \sqrt{q}) a \ln (q / L)-2 J q J(1, a \sqrt{q}) a \ln (q / L)-40 J \sqrt{q} J(0, a \sqrt{q})\right. \\
& +2 J q J(0, a \sqrt{q}) \ln (q / L) J(1, a \sqrt{q}) a-2 q^{2} J(0, a \sqrt{q}) \ln (q / L) J(1, a \sqrt{q}) a
\end{aligned}
$$




$$
\begin{aligned}
& +12 J \sqrt{q} \ln (q / L)+12 J \sqrt{q} J(0, a \sqrt{q})^{2} \ln (q / L)-18 q^{3 / 2} J(0, a \sqrt{q})^{2} \ln (q / L) \\
& -18 q^{3 / 2} \ln (q / L)+20 J \sqrt{q}-20 q^{3 / 2}+20 J \sqrt{q} J(0, a \sqrt{q})^{2}-20 q^{3 / 2} J(0, a \sqrt{q})^{2} \\
& \left.-24 J \sqrt{q} J(0, a \sqrt{q}) \ln (q / L)+36 q^{3 / 2} J(0, a \sqrt{q}) \ln (q / L)+40 q^{3 / 2} J(0, a \sqrt{q})\right) \text {, } \\
& n_{S}^{(2213) G}=\sqrt{J}\left(2 q^{2} \ln (q / L)^{2} J(1, a \sqrt{q}) a+2 J \sqrt{q} \ln (q / L)^{2}-4 q^{3 / 2} \ln (q / L)\right. \\
& -2 J q \ln (q / L)^{2} J(1, a \sqrt{q}) a+2 J q J(0, a \sqrt{q}) \ln (q / L)^{2} J(1, a \sqrt{q}) a \\
& -2 q^{2} J(0, a \sqrt{q}) \ln (q / L)^{2} J(1, a \sqrt{q}) a+2 J \sqrt{q} J(0, a \sqrt{q})^{2} \ln (q / L)^{2} \\
& -3 q^{3 / 2} \ln (q / L)^{2}-3 q^{3 / 2} J(0, a \sqrt{q})^{2} \ln (q / L)^{2}+4 J \sqrt{q} \ln (q / L) \\
& -4 J \sqrt{q} J(0, a \sqrt{q}) \ln (q / L)^{2}+4 J \sqrt{q} J(0, a \sqrt{q})^{2} \ln (q / L) \\
& -4 q^{3 / 2} J(0, a \sqrt{q})^{2} \ln (q / L)+6 q^{3 / 2} J(0, a \sqrt{q}) \ln (q / L)^{2} \\
& \left.-8 J \sqrt{q} J(0, a \sqrt{q}) \ln (q / L)+8 q^{3 / 2} J(0, a \sqrt{q}) \ln (q / L)\right) .
\end{aligned}
$$

In this case, we further obtain that the 222 -factors are

$$
\begin{aligned}
n_{S}^{(2221) G}= & a q\left(3 q^{3 / 2} a \sqrt{J-q} J(0, a \sqrt{q})^{2}+3 J q^{1 / 2} a \sqrt{J-q} J(0, a \sqrt{q})\right. \\
& -3 q^{3 / 2} a \sqrt{J-q} J(0, a \sqrt{q})-3 J q^{1 / 2} a \sqrt{J-q} J(0, a \sqrt{q})^{2} \\
& +4 q^{3 / 2} \sqrt{J-q} J(1, a \sqrt{q})^{2} a-4 q^{1 / 2} \sqrt{J-q} J(1, a \sqrt{q})^{2} a \\
& -6 q \sqrt{J-q} J(1, a \sqrt{q}) J(0, a \sqrt{q})-6 J \sqrt{J-q} J(1, a \sqrt{q}) \\
& +6 J \sqrt{J-q} J(1, a \sqrt{q}) J(0, a \sqrt{q})+6 q \sqrt{J-q} J(1, a \sqrt{q})), \\
n_{S}^{(2222) G}= & a \sqrt{J-q}\left(2 q^{5 / 2} J(1, a \sqrt{q})^{2} a \ln (q / L)-2 q^{3 / 2} J J(1, a \sqrt{q})^{2} a \ln (q / L)\right. \\
& +3 q(5 / 2) a J(0, a \sqrt{q})^{2} \ln (q / L)-3 q^{5 / 2} a J(0, a \sqrt{q}) \ln (q / L) \\
& -3 J q^{3 / 2} a J(0, a \sqrt{q})^{2} \ln (q / L)+3 J q^{3 / 2} a J(0, a \sqrt{q}) \ln (q / L) \\
& +6 J q J(1, a \sqrt{q}) J(0, a \sqrt{q}) \ln (q / L)-6 q^{2} J(1, a \sqrt{q}) J(0, a \sqrt{q}) \ln (q / L) \\
& -6 J q J(1, a \sqrt{q}) \ln (q / L)+6 q^{2} J(1, a \sqrt{q}) \ln (q / L)-10 q J J(1, a \sqrt{q}) \\
& \left.+10 q^{2} J(1, a \sqrt{q})+10 q J J(1, a \sqrt{q}) J(0, a \sqrt{q})-10 q^{2} J(1, a \sqrt{q}) J(0, a \sqrt{q})\right), \\
= & a \sqrt{J-q}\left(q^{5 / 2} a J(0, a \sqrt{q})^{2} \ln (q / L)^{2}-q^{5 / 2} a J(0, a \sqrt{q}) \ln (q / L)^{2}\right. \\
& +J q^{3 / 2} a J(0, a \sqrt{q}) \ln (q / L)^{2}-J q^{3 / 2} a J(0, a \sqrt{q})^{2} \ln (q / L)^{2} \\
& +2 q^{2} J(1, a \sqrt{q}) \ln (q / L)^{2}-2 q^{2} J(1, a \sqrt{q}) J(0, a \sqrt{q}) \ln (q / L)^{2} \\
& -2 J q J(1, a \sqrt{q}) \ln (q / L)^{2}+2 J q J(1, a \sqrt{q}) J(0, a \sqrt{q}) \ln (q / L)^{2} \\
& -4 q J J(1, a \sqrt{q}) \ln (q / L)+4 q J J(1, a \sqrt{q}) J(0, a \sqrt{q}) \ln (q / L) \\
& \left.+4 q^{2} J(1, a \sqrt{q}) \ln (q / L)-4 q^{2} J(1, a \sqrt{q}) J(0, a \sqrt{q}) \ln (q / L)\right) . \\
n_{S}^{(2223) G}(L) &
\end{aligned}
$$

After applying a similar technique, it turns out that the 23 factors are

$$
\begin{aligned}
n_{S}^{(231) G} & =2 p^{3} n_{S}^{(2312) G}+4 p^{4} n_{S}^{(2313) G}, \\
n_{S}^{(232) G} & =p^{3} n_{S}^{(2323) G}+2 p^{4} n_{S}^{(2324) G},
\end{aligned}
$$

where the 230 and 231 sub-factors are expressed as

$$
\begin{aligned}
& n_{S}^{(230) G}=8 J p^{3} \sqrt{q} \sqrt{J-q}(2 J(0, a \sqrt{q})+2 p J(0, a \sqrt{q}) \ln (q / L) \\
& \left.-p J(0, a \sqrt{q})^{2} \ln (q / L)-J(0, a \sqrt{q})^{2}-p \ln (q / L)-1\right), \\
& n_{S}^{(2312) G}=\sqrt{J}\left(2 J q J(1, a \sqrt{q}) a+2 q^{2} J(1, a \sqrt{q}) a J(0, a \sqrt{q})+2 J \sqrt{q} l(p)^{2}\right. \\
& -2 J q J(1, a \sqrt{q}) a J(0, a \sqrt{q})-2 q^{2} J(1, a \sqrt{q}) a+2 J \sqrt{q} J(0, a \sqrt{q})^{2} \\
& -3 q^{3 / 2}\left((q / L)^{p}\right)^{3}-3 q^{3 / 2} J(0, a \sqrt{q})^{2}-4 J \sqrt{q} J(0, a \sqrt{q}) \\
& \left.+6 q^{3 / 2} J(0, a \sqrt{q})\right) \text {, }
\end{aligned}
$$




$$
\begin{aligned}
n_{S}^{(2313) G}= & \sqrt{q J}\left(2 J \ln (q / L)+2 J J(0, a \sqrt{q})^{2} \ln (q / L)-3 q J(0, a \sqrt{q})^{2} \ln (q / L)\right. \\
& -3 q \ln (q / L)-4 J J(0, a \sqrt{q}) \ln (q / L)-4 q+4 J+4 J J(0, a \sqrt{q})^{2} \\
& -4 q J(0, a \sqrt{q})^{2}+6 q J(0, a \sqrt{q}) \ln (q / L)+8 q J(0, a \sqrt{q}) \\
& -8 J J(0, a \sqrt{q})) .
\end{aligned}
$$

While, the 232 factors come as per the following identifications

$$
\begin{aligned}
n_{S}^{(2323) G}= & a f^{2} \sqrt{J-q}\left(q^{5 / 2} a J(0, a \sqrt{q})^{2}+J q^{3 / 2} a J(0, a \sqrt{q})-J q^{3 / 2} a J(0, a \sqrt{q})^{2}\right. \\
& -q^{5 / 2} a J(0, a \sqrt{q})+2 q^{5 / 2} J(1, a \sqrt{q})^{2} a-2 q^{3 / 2} J J(1, a \sqrt{q})^{2} a \\
& -2 q^{2} J(1, a \sqrt{q}) J(0, a \sqrt{q})-2 J q J(1, a \sqrt{q}) \\
& \left.+2 J q J(1, a \sqrt{q}) J(0, a \sqrt{q})+2 p^{3} q^{2} J(1, a \sqrt{q})\right) \\
n_{S}^{(2324) G}= & a f^{2} \sqrt{J-q}\left(q^{5 / 2} J(1, a \sqrt{q})^{2} a \ln (q / L)+q^{5 / 2} a J(0, a \sqrt{q})^{2} \ln (q / L)\right. \\
& -q^{5 / 2} a J(0, a \sqrt{q}) \ln (q / L)-q^{3 / 2} J J(1, a \sqrt{q})^{2} a \ln (q / L) \\
& +J q^{3 / 2} a J(0, a \sqrt{q}) \ln (q / L)-J q^{3 / 2} a J(0, a \sqrt{q})^{2} \ln (q / L) \\
& +2 J q J(1, a \sqrt{q}) J(0, a \sqrt{q}) \ln (q / L)-2 J q J(1, a \sqrt{q}) \ln (q / L) \\
& -2 q^{2} J(1, a \sqrt{q}) J(0, a \sqrt{q}) \ln (q / L)+4 q^{2} J(1, a \sqrt{q}) \\
& +4 q J J(0, a \sqrt{q}) J(1, a \sqrt{q})-4 q^{2} J(1, a \sqrt{q}) J(0, a \sqrt{q}) \\
& \left.+2 q^{2} J(1, a \sqrt{q}) \ln (q / L)-4 q J J(1, a \sqrt{q})\right) .
\end{aligned}
$$

We notice further that the factors of the $l(p)^{3}$-terms can be expressed as

$$
n_{S}^{(3) G}=n_{S}^{(30) G}+(q / L)^{p} n_{S}^{(31) G}+(q / L)^{2 p} n_{S}^{(32) G}+\left((q / L)^{3 p} n_{S}^{(33) G}\right) .
$$

Following similar factorizations, we have the following terms

$$
\begin{aligned}
n_{S}^{(30) G} & =n_{S}^{(300) G}+n_{S}^{(301) G} f+n_{S}^{(302) G} f^{2}, \\
n_{S}^{(31) G} & =n_{S}^{(310) G}+n_{S}^{(311) G} f+n_{S}^{(312) G} f^{2}, \\
n_{S}^{(32) G} & =n_{S}^{(320) G}+n_{S}^{(321) G} f+n_{S}^{(322) G} f^{2}, \\
n_{S}^{(33) G} & =n_{S}^{(330) G}+n_{S}^{(331) G} f+n_{S}^{(332) G} f^{2},
\end{aligned}
$$

which factor nicely. As per the expectation, we find that the 30 -factors are

$$
\begin{aligned}
n_{S}^{(300) G} & =4 \sqrt{q} \sqrt{J-q} J\left(1-2 J(0, a \sqrt{q})+J(0, a \sqrt{q})^{2}\right), \\
n_{S}^{(301) G} & =4 a \sqrt{J} J(1, a \sqrt{q})\left(q^{2}-q^{2} J(0, a \sqrt{q})-J q+J q J(0, a \sqrt{q})\right), \\
n_{S}^{(302) G} & =f^{2} a^{2} q^{3 / 2} \sqrt{J-q} J(1, a \sqrt{q})^{2}(J-q) .
\end{aligned}
$$

Similarly, the 31-factors are obtained as

$$
\begin{aligned}
n_{S}^{(310) G}= & 12 \sqrt{q} \sqrt{J-q} p J\left(1+J(0, a \sqrt{q})^{2}-2 J(0, a \sqrt{q})\right), \\
n_{S}^{(311) G}= & 12 q p a \sqrt{J}(q J(1, a \sqrt{q})-q J(1, a \sqrt{q}) J(0, a \sqrt{q}) \\
& -J J(1, a \sqrt{q})+J J(1, a \sqrt{q}) J(0, a \sqrt{q})), \\
n_{S}^{(312) G}= & 3 q^{3 / 2} \sqrt{J-q} f^{2} a^{2} J(1, a \sqrt{q})^{2} p(J-q) .
\end{aligned}
$$

The 32-factors reduce to 


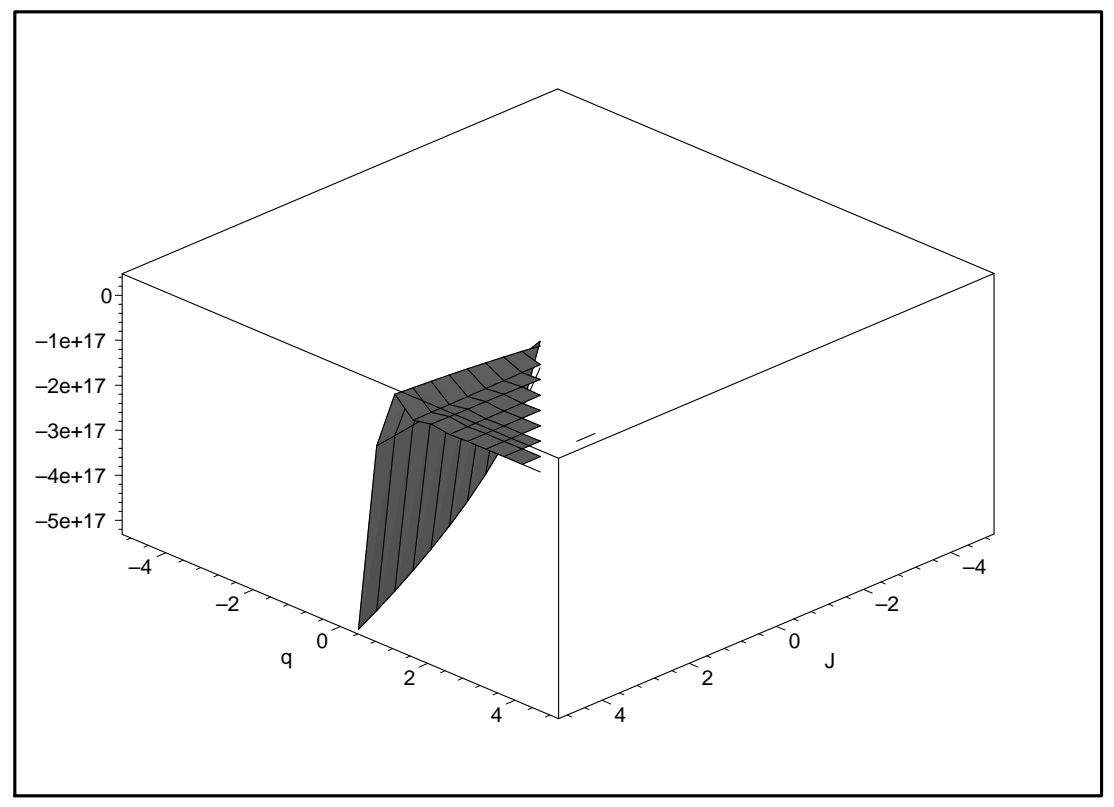

Figure 16: The surface minor $p_{S}^{G}$ plotted as a function of the scale and angular momentum, viz., $q, J$, describing the nature of the stability of the $q p$-surface for general massive rotating quarkonia.

$$
\begin{aligned}
n_{S}^{(320) G}= & 12 \sqrt{q} \sqrt{J-q} p^{2} J\left(1+J(0, a \sqrt{q})^{2}-2 J(0, a \sqrt{q})\right), \\
n_{S}^{(321) G}= & 12 a q p^{2} \sqrt{J}(q J(1, a \sqrt{q})-q J(1, a \sqrt{q}) J(0, a \sqrt{q}) \\
& +J J(1, a \sqrt{q}) J(0, a \sqrt{q})-J J(1, a \sqrt{q})), \\
n_{S}^{(322) G}= & 3 q^{3 / 2} \sqrt{J-q} f^{2} a^{2} J(1, a \sqrt{q})^{2} p^{2}(J-q) .
\end{aligned}
$$

Finally, it follows that the 33 -factors are given by

$$
\begin{aligned}
& n_{S}^{(330) G}=4 \sqrt{q} \sqrt{J-q} p^{3} J\left(J(0, a \sqrt{q})^{2}-2 J J(0, a \sqrt{q})+1\right), \\
& n_{S}^{(331) G}=4 a q p^{3} \sqrt{J} J(1, a \sqrt{q})(q-q J(0, a \sqrt{q})-J+J J(0, a \sqrt{q})), \\
& n_{S}^{(332) G}=+q^{3 / 2} \sqrt{J-q} f^{2} a^{2} J(1, a \sqrt{q})^{2} p^{3}(J-q) .
\end{aligned}
$$

For the general quarkonia with $\{q, p, J\}$ fluctuating, the stability of the $q p$-surface requires that the principle minor $p_{S}^{G}$ remains positive on $\left(M_{3}, g\right)$. Correspondingly, this leads to the constraint that the $\{q, p, J\}$ satisfy

$$
n_{S}^{(0) G}+n_{g}^{(1) G} l(p)+n_{S}^{(2) G} l(p)^{2}+n_{S}^{(3) G} l(p)^{3}<0 .
$$

The three dimensional graphical views of the surface minor $p_{S}^{G}$ of generic quarkonia is depicted in the Fig.(16). For the limiting small scale $q \rightarrow 0^{+}$, the graphical view of the surface minor $p_{S}^{G}$ shows a large negative minima of the order of the height $-10^{17}$, which occurs in the regime of a large angular momentum $J \rightarrow 5^{-}$. When all the parameters are allowed to fluctuate and $b$ is an arbitrary real number, the exact intrinsic geometric characterization of the minor is explicitly obtained in the Eqn.(59), which forms the basis for the stability of the $q p$-surface.

After considering the contributions of the fluctuations to the strongly coupled massive rotating quarkonia, we obtain the following general expressions for the determinant of the metric tensor

$$
\|g\|=-\frac{p f(-1+J(0, a \sqrt{q}))}{8 b^{3} l(p)^{6} \exp (3 l(p)) q^{5 / 2} J^{3 / 2}(J-q)^{3 / 2}}\left(n_{g}^{(0) G}+n_{g}^{(1) G} l(p)+n_{g}^{(2) G} l(p)^{2}+n_{g}^{(3) G} l(p)^{3}\right),
$$


where the $n_{g}^{(0) G}$-term factorizes as

$$
n_{g}^{(0) G}=\left((q / L)^{p}\right)^{3}\left(8 p^{4} n_{g}^{(04) G}+8 p^{5} n_{g}^{(05) G}+8 p^{6} n_{g}^{(06) G}\right) .
$$

After simplification, we obtain the sub-factorizations

$$
\begin{aligned}
n_{g}^{(04) G} & =n_{g}^{(040) G}+f n_{g}^{(041) G}, \\
n_{g}^{(05) G} & =n_{g}^{(050) G}+f n_{g}^{(051) G}, \\
n_{g}^{(06) G} & =n_{g}^{(060) G}+f n_{g}^{(061) G} .
\end{aligned}
$$

As per our computation, the $\left\{n_{g}^{(040) G}, n_{g}^{(041) G}, n_{g}^{(050) G}, n_{g}^{(051) G}, n_{g}^{(060) G}, n_{g}^{(061) G}\right\}$ are expressed as

$$
\begin{aligned}
n_{g}^{(040) G}= & \sqrt{J} \sqrt{J-q} \sqrt{q}\left(2 J(0, a \sqrt{q})-J(0, a \sqrt{q})^{2}-1\right), \\
n_{g}^{(041) G}= & \left(q^{3 / 2}+q^{3 / 2} J(0, a \sqrt{q})^{2}-2 q^{3 / 2} J(0, a \sqrt{q})\right. \\
& \left.-2 J \sqrt{q} J(0, a \sqrt{q})^{2}-2 J \sqrt{q}+4 J \sqrt{q} J(0, a \sqrt{q})\right), \\
n_{g}^{(050) G}=\quad & \sqrt{J} \sqrt{J-q} \sqrt{q}(4 J(0, a \sqrt{q}) \ln (q / L)+6 J(0, a \sqrt{q}) \\
& \left.-2 \ln (q / L)-2 J(0, a \sqrt{q})^{2} \ln (q / L)-3-3 J(0, a \sqrt{q})^{2}\right), \\
n_{g}^{(051) G}=\quad & 3 q^{3 / 2}+3 q^{3 / 2} J(0, a \sqrt{q})^{2}+2 q^{3 / 2} J(0, a \sqrt{q})^{2} \ln (q / L) \\
+ & 2 q^{3 / 2} \ln (q / L)-6 q^{3 / 2} J(0, a \sqrt{q})+8 J \sqrt{q} J(0, a \sqrt{q}) \ln (q / L) \\
+ & 12 J \sqrt{q} J(0, a \sqrt{q})-4 J \sqrt{q} \ln (q / L)-6 J \sqrt{q} J(0, a \sqrt{q})^{2} \\
- & 4 J \sqrt{q} J(0, a \sqrt{q})^{2} \ln (q / L)-4 q^{3 / 2} J(0, a \sqrt{q}) \ln (q / L)-6 J \sqrt{q},
\end{aligned}
$$

and

$$
\begin{aligned}
n_{g}^{(060) G}= & \sqrt{J} \sqrt{J-q} \sqrt{q}\left(4 J(0, a \sqrt{q}) \ln (q / L)+2 J(0, a \sqrt{q}) \ln (q / L)^{2}\right. \\
& -2 \ln (q / L)-J(0, a \sqrt{q})^{2} \ln (q / L)^{2}-2 J(0, a \sqrt{q})^{2} \ln (q / L) \\
& \left.-\ln (q / L)^{2}\right) \\
n_{g}^{(061) G}= & q^{3 / 2} \ln (q / L)^{2}+q^{3 / 2} J(0, a \sqrt{q})^{2} \ln (q / L)^{2}+2 q^{3 / 2} \ln (q / L) \\
& +2 q^{3 / 2} J(0, a \sqrt{q})^{2} \ln (q / L)-2 J \sqrt{q} J(0, a \sqrt{q})^{2} \ln (q / L)^{2} \\
& -2 q^{3 / 2} J(0, a \sqrt{q}) \ln (q / L)^{2}-4 q^{3 / 2} J(0, a \sqrt{q}) \ln (q / L) \\
& -4 J \sqrt{q} J(0, a \sqrt{q})^{2} \ln (q / L)+4 J \sqrt{q} J(0, a \sqrt{q}) \ln (q / L)^{2} \\
& -2 J \sqrt{q} \ln (q / L)^{2}+8 J \sqrt{q} J(0, a \sqrt{q}) \ln (q / L) .
\end{aligned}
$$

We find that the $l(p)$ terms factorize as follows

$$
n_{g}^{(1) G}=\left((q / L)^{p}\right)^{2} n_{g}^{(12) G}+\left((q / L)^{p}\right)^{3} n_{g}^{(13) G}
$$

with the following sub-factorizations

$$
\begin{aligned}
& n_{g}^{(12) G}=n_{g}^{(120) G}+f n_{g}^{(121) G}, \\
& n_{g}^{(13) G}=n_{g}^{(130) G}+f n_{g}^{(131) G} .
\end{aligned}
$$

The factors of the 12-components are given by 


$$
\begin{aligned}
n_{g}^{(120) G} & =n_{g}^{(1202) G} p^{2}+n_{g}^{(1203) G} p^{3}+n_{g}^{(1204) G} p^{4}, \\
n_{g}^{(121) G} & =n_{g}^{(1212) G} p^{2}+n_{g}^{(1213) G} p^{3}+n_{g}^{(1214) G} p^{4}+n_{g}^{(1215) G} p^{5}
\end{aligned}
$$

with the following sub-factorizations

$$
\begin{aligned}
n_{g}^{(1202) G}= & 2 \sqrt{J} \sqrt{J-q}\left(a^{2} q^{3 / 2} J(0, a \sqrt{q})^{2}-a^{2} q^{3 / 2} J(0, a \sqrt{q})+6 J(1, a \sqrt{q}) a q\right. \\
& \left.-6 J(1, a \sqrt{q}) a q J(0, a \sqrt{q})-8 \sqrt{q} J(0, a \sqrt{q})^{2}-8 \sqrt{q}+16 \sqrt{q} J(0, a \sqrt{q})\right), \\
n_{g}^{(1203) G}= & 4 \sqrt{J} \sqrt{J-q}\left(a^{2} q^{3 / 2} J(0, a \sqrt{q})^{2} \ln (q / L)-a^{2} q^{3 / 2} J(0, a \sqrt{q}) \ln (q / L)\right. \\
& +6 J(1, a \sqrt{q}) a q+6 J(1, a \sqrt{q}) a q \ln (q / L)-6 J(1, a \sqrt{q}) a q J(0, a \sqrt{q}) \ln (q / L) \\
& -6 q J(1, a \sqrt{q}) a J(0, a \sqrt{q})-8 \sqrt{q} \ln (q / L)-8 \sqrt{q} J(0, a \sqrt{q})^{2} \ln (q / L) \\
& \left.-8 \sqrt{q} J(0, a \sqrt{q})^{2}-8 \sqrt{q}+16 \sqrt{q} J(0, a \sqrt{q})+16 \sqrt{q} J(0, a \sqrt{q}) \ln (q / L)\right), \\
n_{g}^{(1204) G}= & 2 \sqrt{J} \sqrt{J-q}\left(a^{2} q^{3 / 2} J(0, a \sqrt{q})^{2} \ln (q / L)^{2}-a^{2} q^{3 / 2} J(0, a \sqrt{q}) \ln (q / L)^{2}\right. \\
& -6 J(1, a \sqrt{q}) a q J(0, a \sqrt{q}) \ln (q / L)^{2}+6 J(1, a \sqrt{q}) a q \ln (q / L)^{2}+8 \sqrt{q} \\
& -8 \sqrt{q} \ln (q / L)-8 J(1, a \sqrt{q}) a q J(0, a \sqrt{q}) \ln (q / L)+8 \sqrt{q} J(0, a \sqrt{q})^{2} \\
& -8 \sqrt{q} \ln (q / L)^{2}-8 \sqrt{q} J(0, a \sqrt{q})^{2} \ln (q / L)^{2}-8 \sqrt{q} J(0, a \sqrt{q})^{2} \ln (q / L) \\
& +8 q \ln (q / L) J(1, a \sqrt{q}) a-16 \sqrt{q} J(0, a \sqrt{q})+16 \sqrt{q} J(0, a \sqrt{q}) \ln (q / L)^{2} \\
+ & 16 \sqrt{q} J(0, a \sqrt{q}) \ln (q / L)) .
\end{aligned}
$$

The 121-factors turn out to be

$$
\begin{aligned}
& n_{g}^{(1212) G}=a q\left(2 J q^{1 / 2} J(1, a \sqrt{q})^{2} a+2 a q^{3 / 2} J(0, a \sqrt{q})-2 a q^{3 / 2} J(0, a \sqrt{q})^{2}\right. \\
& +4 q J(1, a \sqrt{q}) J(0, a \sqrt{q})-4 J(1, a \sqrt{q}) q+4 J a q^{1 / 2} J(0, a \sqrt{q})^{2} \\
& -4 J a q^{1 / 2} J(0, a \sqrt{q})-8 J J(1, a \sqrt{q}) J(0, a \sqrt{q})+8 J J(1, a \sqrt{q}) \\
& \left.-q^{3 / 2} J(1, a \sqrt{q})^{2} a\right) \text {, } \\
& n_{g}^{(1213) G}=2\left(-q^{5 / 2} J(1, a \sqrt{q})^{2} a^{2} \ln (q / L)+2 a^{2} q^{5 / 2} J(0, a \sqrt{q}) \ln (q / L)\right. \\
& -2 a^{2} q^{5 / 2} J(0, a \sqrt{q})^{2} \ln (q / L)+2 J q^{3 / 2} J(1, a \sqrt{q})^{2} a^{2} \ln (q / L) \\
& +4 J(1, a \sqrt{q}) a q^{2} J(0, a \sqrt{q}) \ln (q / L)+4 J a^{2} q^{3 / 2} J(0, a \sqrt{q})^{2} \ln (q / L) \\
& -4 q^{3 / 2} J(0, a \sqrt{q})^{2}-4 J(1, a \sqrt{q}) a q^{2} \ln (q / L)-6 J(1, a \sqrt{q}) a q^{2} \\
& -4 J a^{2} q^{3 / 2} J(0, a \sqrt{q}) \ln (q / L)-4 q^{3 / 2}+6 J(1, a \sqrt{q}) a q^{2} J(0, a \sqrt{q}) \\
& +8 J \sqrt{q}+8 q^{3 / 2} J(0, a \sqrt{q})-8 J J(1, a \sqrt{q}) a q J(0, a \sqrt{q}) \ln (q / L) \\
& +8 J J(1, a \sqrt{q}) a q \ln (q / L)+8 J \sqrt{q} J(0, a \sqrt{q})^{2}+12 J J(1, a \sqrt{q}) a q \\
& -12 J J(1, a \sqrt{q}) a q J(0, a \sqrt{q})-16 J \sqrt{q} J(0, a \sqrt{q})) \text {, } \\
& n_{g}^{(1214) G}=2\left(-q^{5 / 2} J(1, a \sqrt{q})^{2} a^{2} \ln (q / L)^{2}+2 J q^{3 / 2} J(1, a \sqrt{q})^{2} a^{2} \ln (q / L)^{2}\right. \\
& +2 a^{2} q^{5 / 2} J(0, a \sqrt{q}) \ln (q / L)^{2}-2 a^{2} q^{5 / 2} J(0, a \sqrt{q})^{2} \ln (q / L)^{2} \\
& +4 J(1, a \sqrt{q}) a q^{2} J(0, a \sqrt{q}) \ln (q / L)^{2}-4 a q^{2} J(1, a \sqrt{q}) \ln (q / L)^{2} \\
& +4 J a^{2} q^{3 / 2} J(0, a \sqrt{q})^{2} \ln (q / L)^{2}-4 J a^{2} q^{3 / 2} J(0, a \sqrt{q}) \ln (q / L)^{2} \\
& +8 J(1, a \sqrt{q}) a q^{2} J(0, a \sqrt{q}) \ln (q / L)-8 J(1, a \sqrt{q}) a q^{2} \ln (q / L) \\
& +8 J a q J(1, a \sqrt{q}) \ln (q / L)^{2}-8 J J(1, a \sqrt{q}) a q J(0, a \sqrt{q}) \ln (q / L)^{2} \\
& -16 q^{3 / 2} J(0, a \sqrt{q})^{2} \ln (q / L)-16 J J(1, a \sqrt{q}) a q J(0, a \sqrt{q}) \ln (q / L) \\
& +16 J J(1, a \sqrt{q}) a q \ln (q / L)-16 q^{3 / 2} \ln (q / L)-28 q^{3 / 2} J(0, a \sqrt{q})^{2} \\
& -28 q^{3 / 2}+32 J \sqrt{q} \ln (q / L)+32 J \sqrt{q} J(0, a \sqrt{q})^{2} \ln (q / L)+56 J \sqrt{q} \\
& +32 q^{3 / 2} J(0, a \sqrt{q}) \ln (q / L)+56 q^{3 / 2} J(0, a \sqrt{q})+56 J \sqrt{q} J(0, a \sqrt{q})^{2} \\
& -64 J \sqrt{q} J(0, a \sqrt{q}) \ln (q / L)-112 J \sqrt{q} J(0, a \sqrt{q}) \text {, }
\end{aligned}
$$




$$
\begin{aligned}
n_{g}^{(1215) G=} & 4 \sqrt{q} \ln (q / L)\left(-q \ln (q / L)-q J(0, a \sqrt{q})^{2} \ln (q / L)+2 J \ln (q / L)\right. \\
& -2 q-2 q J(0, a \sqrt{q})^{2}+2 q J(0, a \sqrt{q}) \ln (q / L)+2 J J(0, a \sqrt{q})^{2} \ln (q / L) \\
& -4 J J(0, a \sqrt{q}) \ln (q / L)+4 J J(0, a \sqrt{q})^{2}+4 J+4 q J(0, a \sqrt{q}) \\
& -8 J J(0, a \sqrt{q})) .
\end{aligned}
$$

Similarly, the factors of 13-components are given as

$$
\begin{aligned}
& n_{g}^{(130) G}=n_{g}^{(1303) G} p^{3}+n_{g}^{(1304) G} p^{4}+n_{g}^{(1305) G} p^{5}, \\
& n_{g}^{(131) G}=n_{g}^{(1313) G} p^{3}+n_{g}^{(1314) G} p^{4}+n_{g}^{(1315) G} p^{5}+n_{g}^{(1316) G} p^{6} .
\end{aligned}
$$

The 130-sub-factors turn out to be

$$
\begin{aligned}
n_{g}^{(1303) G}= & 2 \sqrt{J} \sqrt{J-q}\left(a^{2} q^{3 / 2} J(0, a \sqrt{q})^{2}-a^{2} q^{3 / 2} J(0, a \sqrt{q})+6 J(1, a \sqrt{q}) a q\right. \\
& \left.-6 a q J(1, a \sqrt{q}) J(0, a \sqrt{q})-8 \sqrt{q}-8 \sqrt{q} J(0, a \sqrt{q})^{2}+16 \sqrt{q} J(0, a \sqrt{q})\right), \\
n_{g}^{(1304) G}= & 4 \sqrt{J} \sqrt{J-q}\left(a^{2} q^{3 / 2} J(0, a \sqrt{q})^{2} \ln (q / L)-a^{2} q^{3 / 2} J(0, a \sqrt{q}) \ln (q / L)\right. \\
& +6 q J(1, a \sqrt{q}) a-6 a q J(1, a \sqrt{q}) J(0, a \sqrt{q}) \ln (q / L)+6 J(1, a \sqrt{q}) a q \ln (q / L) \\
& -6 q J(1, a \sqrt{q}) a J(0, a \sqrt{q})-8 \sqrt{q}-8 \sqrt{q} J(0, a \sqrt{q})^{2} \ln (q / L)-8 \sqrt{q} \ln (q / L) \\
& \left.-8 \sqrt{q} J(0, a \sqrt{q})^{2}+16 \sqrt{q} J(0, a \sqrt{q}) \ln (q / L)+16 \sqrt{q} J(0, a \sqrt{q})\right), \\
n_{g}^{(1305) G}= & 2 \sqrt{J} \sqrt{J-q}\left(a^{2} q^{3 / 2} J(0, a \sqrt{q})^{2} \ln (q / L)^{2}-a^{2} q^{3 / 2} J(0, a \sqrt{q}) \ln (q / L)^{2}\right. \\
& +6 J(1, a \sqrt{q}) a q \ln (q / L)^{2}-6 J(1, a \sqrt{q}) a q J(0, a \sqrt{q}) \ln (q / L)^{2}-8 \sqrt{q} \ln (q / L) \\
& +8 \sqrt{q} J(0, a \sqrt{q})^{2}-8 \sqrt{q} J(0, a \sqrt{q})^{2} \ln (q / L)^{2}+8 \sqrt{q}-8 \sqrt{q} \ln (q / L)^{2} \\
& -8 q J(0, a \sqrt{q}) \ln (q / L) J(1, a \sqrt{q}) a+8 q \ln (q / L) J(1, a \sqrt{q}) a \\
& -8 \sqrt{q} J(0, a \sqrt{q})^{2} \ln (q / L)+16 \sqrt{q} J(0, a \sqrt{q}) \ln (q / L)^{2} \\
& +16 \sqrt{q} J(0, a \sqrt{q}) \ln (q / L)-16 \sqrt{q} J(0, a \sqrt{q})) .
\end{aligned}
$$

The 131-factors take the following explicit expressions

$$
\begin{aligned}
n_{g}^{(1313) G}= & a q\left(-q^{3 / 2} J(1, a \sqrt{q})^{2} a+2 J q^{1 / 2} J(1, a \sqrt{q})^{2} a+2 a q^{3 / 2} J(0, a \sqrt{q})\right. \\
& -2 a q^{3 / 2} J(0, a \sqrt{q})^{2}-4 J(1, a \sqrt{q}) q+4 J(1, a \sqrt{q}) q J(0, a \sqrt{q}) \\
& -4 J a q^{1 / 2} J(0, a \sqrt{q})+4 J a q^{1 / 2} J(0, a \sqrt{q})^{2}+8 J J(1, a \sqrt{q}) \\
& -8 J J(1, a \sqrt{q}) J(0, a \sqrt{q})), \\
n_{g}^{(1314) G}= & 2\left(-q^{5 / 2} J(1, a \sqrt{q})^{2} a^{2} \ln (q / L)-2 q^{3 / 2}-2 q^{3 / 2} J(0, a \sqrt{q})^{2}\right. \\
& +2 a^{2} q^{5 / 2} J(0, a \sqrt{q}) \ln (q / L)-2 a^{2} q^{5 / 2} J(0, a \sqrt{q})^{2} \ln (q / L) \\
& +2 J q^{3 / 2} J(1, a \sqrt{q})^{2} a^{2} \ln (q / L)+4 J \sqrt{q}+4 J \sqrt{q} J(0, a \sqrt{q})^{2} \\
& +4 J a^{2} q^{3 / 2} J(0, a \sqrt{q})^{2} \ln (q / L)+4 a q^{2} J(1, a \sqrt{q}) J(0, a \sqrt{q}) \ln (q / L) \\
& -4 J(1, a \sqrt{q}) a q^{2} \ln (q / L)-4 J a^{2} q^{3 / 2} J(0, a \sqrt{q}) \ln (q / L) \\
& +4 q^{3 / 2} J(0, a \sqrt{q})-6 J(1, a \sqrt{q}) a q^{2}+6 J(1, a \sqrt{q}) a q^{2} J(0, a \sqrt{q}) \\
& -8 J \sqrt{q} J(0, a \sqrt{q})+8 J J(1, a \sqrt{q}) a q \ln (q / L)+12 J J(1, a \sqrt{q}) a q \\
& -8 J a q J(1, a \sqrt{q}) J(0, a \sqrt{q}) \ln (q / L)-12 J J(1, a \sqrt{q}) a q J(0, a \sqrt{q})), \\
= & -q^{5 / 2} J(1, a \sqrt{q})^{2} a^{2} \ln (q / L)^{2}+2 a^{2} q^{5 / 2} J(0, a \sqrt{q}) \ln (q / L)^{2} \\
& \left.-2 a^{2} q^{5 / 2} J(0, a \sqrt{q})^{2} \ln (q / L)^{2}\right)+2 J q^{3 / 2} J(1, a \sqrt{q})^{2} a^{2} \ln (q / L)^{2} \\
& +4 J(1, a \sqrt{q}) a q^{2} J(0, a \sqrt{q}) \ln (q / L)^{2}-4 J(1, a \sqrt{q}) a q^{2} \ln (q / L)^{2} \\
& +4 J a^{2} q^{3 / 2} J(0, a \sqrt{q})^{2} \ln (q / L)^{2}-4 J a^{2} q^{3 / 2} J(0, a \sqrt{q}) \ln (q / L)^{2} \\
& +8 J J(1, a \sqrt{q}) a q \ln (q / L)^{2}-8 J J(1, a \sqrt{q}) a q J(0, a \sqrt{q}) \ln (q / L)^{2} \\
& +8 J(1, a \sqrt{q}) a q^{2} J(0, a \sqrt{q}) \ln (q / L)-8 J(1, a \sqrt{q}) a q^{2} \ln (q / L) \\
n_{g}^{(1315) G}(L) &
\end{aligned}
$$




$$
\begin{aligned}
& -8 q^{3 / 2} J(0, a \sqrt{q})^{2} \ln (q / L)-8 q^{3 / 2} \ln (q / L)+16 J \sqrt{q} \ln (q / L) \\
& +16 q^{3 / 2} J(0, a \sqrt{q}) \ln (q / L)+16 J J(1, a \sqrt{q}) a q \ln (q / L)-16 q^{3 / 2} \\
& -16 J J(1, a \sqrt{q}) a q J(0, a \sqrt{q}) \ln (q / L)+16 J \sqrt{q} J(0, a \sqrt{q})^{2} \ln (q / L) \\
& -16 q^{3 / 2} J(0, a \sqrt{q})^{2}+32 J \sqrt{q} J(0, a \sqrt{q})^{2}+32 q^{3 / 2} J(0, a \sqrt{q}) \\
& +32 J \sqrt{q}-32 J \sqrt{q} J(0, a \sqrt{q}) \ln (q / L)-64 J \sqrt{q} J(0, a \sqrt{q}), \\
n_{g}^{(1316) G}= & -32 J \sqrt{q} \ln (q / L) .
\end{aligned}
$$

It follows that the $l(p)^{2}$ terms factorize as

$$
n_{g}^{(2) G}=(q / L)^{p} n_{g}^{(21) G}+\left((q / L)^{p}\right)^{2} n_{g}^{(22) G}+\left((q / L)^{p}\right)^{3} n_{g}^{(23) G},
$$

where the sub-factorizations take the following forms

$$
\begin{aligned}
n_{g}^{(21) G} & =n_{g}^{(210) G}+f n_{g}^{(211) G}, \\
n_{g}^{(22) G} & =n_{g}^{(220) G}+f n_{g}^{(221) G}, \\
n_{g}^{(23) G} & =n_{g}^{(230) G}+f n_{g}^{(231) G} .
\end{aligned}
$$

Explicitly, we find that the individual factors appear as

$$
\begin{aligned}
& n_{g}^{(210) G}=n_{g}^{(2101) G} p+n_{g}^{(2102) G} p^{2}+n_{g}^{(2103) G} p^{3}, \\
& n_{g}^{(211) G}=n_{g}^{(2111) G} p+n_{g}^{(2112) G} p^{2}+n_{g}^{(2113) G} p^{3} .
\end{aligned}
$$

The 210-factors are given by

$$
\begin{aligned}
n_{g}^{(2101) G}= & 4 \sqrt{J} \sqrt{J-q}\left(a^{2} q^{3 / 2} J(0, a \sqrt{q})-q^{3 / 2} J(1, a \sqrt{q})^{2} a^{2}-a^{2} q^{3 / 2} J(0, a \sqrt{q})^{2}\right. \\
& -2 J(1, a \sqrt{q}) a q+2 J(1, a \sqrt{q}) a q J(0, a \sqrt{q})+4 \sqrt{q}+4 \sqrt{q} J(0, a \sqrt{q})^{2} \\
& -8 \sqrt{q} J(0, a \sqrt{q}))) \\
n_{g}^{(2102) G}= & 4 \sqrt{J} \sqrt{J-q}\left(a^{2} q^{3 / 2} J(0, a \sqrt{q}) \ln (q / L)-a^{2} q^{3 / 2} J(0, a \sqrt{q})^{2} \ln (q / L)\right. \\
& +6 J(1, a \sqrt{q}) a q J(0, a \sqrt{q}) \ln (q / L)+6 p^{2} J(1, a \sqrt{q}) a q J(0, a \sqrt{q}) \\
& -6 J(1, a \sqrt{q}) a q-6 J(1, a \sqrt{q}) a q \ln (q / L)+8 \sqrt{q} \ln (q / L) \\
& +8 \sqrt{q} J(0, a \sqrt{q})^{2} \ln (q / L)+10 \sqrt{q}+10 \sqrt{q} J(0, a \sqrt{q})^{2} \\
& -16 \sqrt{q} J(0, a \sqrt{q}) \ln (q / L)-20 \sqrt{q} J(0, a \sqrt{q})) \\
= & 2 \sqrt{J} \sqrt{J-q}\left(q^{3 / 2} J(1, a \sqrt{q})^{2} a^{2} \ln (q / L)^{2}+4 J(1, a \sqrt{q}) a q J(0, a \sqrt{q}) \ln (q / L)^{2}\right. \\
& +4 q J(0, a \sqrt{q}) \ln (q / L) J(1, a \sqrt{q}) a+4 \sqrt{q} J(0, a \sqrt{q})^{2}+4 \sqrt{q} \ln (q / L)^{2} \\
& -4 q \ln (q / L) J(1, a \sqrt{q}) a-4 J(1, a \sqrt{q}) a q \ln (q / L)^{2}-8 \sqrt{q} J(0, a \sqrt{q}) \\
& +4 \sqrt{q}+4 \sqrt{q} J(0, a \sqrt{q})^{2} \ln (q / L)^{2}+8 \sqrt{q} \ln (q / L)+8 \sqrt{q} J(0, a \sqrt{q})^{2} \ln (q / L) \\
& \left.-8 \sqrt{q} J(0, a \sqrt{q}) \ln (q / L)^{2}-16 \sqrt{q} J(0, a \sqrt{q}) \ln (q / L)\right) .
\end{aligned}
$$

While, the 211-factors are given as

$$
\begin{aligned}
n_{g}^{(2111) G}= & 2 a\left(a q^{5 / 2} J(0, a \sqrt{q})^{2}-a q^{5 / 2} J(0, a \sqrt{q})+q^{5 / 2} J(1, a \sqrt{q})^{2} a\right. \\
& +2 J(1, a \sqrt{q}) q^{2}-2 J(1, a \sqrt{q}) q^{2} J(0, a \sqrt{q})-2 J q^{3 / 2} J(1, a \sqrt{q})^{2} a \\
& -2 J a q^{3 / 2} J(0, a \sqrt{q})^{2}+2 J p a q^{3 / 2} J(0, a \sqrt{q})-4 J J(1, a \sqrt{q}) q \\
& +4 J J(1, a \sqrt{q}) q J(0, a \sqrt{q})) \\
n_{g}^{(2112) G}= & 2 a\left(q^{5 / 2} J(1, a \sqrt{q})^{2} a \ln (q / L)+2 a q^{5 / 2} J(0, a \sqrt{q})^{2} \ln (q / L)\right.
\end{aligned}
$$




$$
\begin{aligned}
& -2 J q^{3 / 2} J(1, a \sqrt{q})^{2} a \ln (q / L)-2 a q^{5 / 2} J(0, a \sqrt{q}) \ln (q / L) \\
& +4 J a q^{3 / 2} J(0, a \sqrt{q}) \ln (q / L)+4 J(1, a \sqrt{q}) q^{2} \ln (q / L) \\
& -4 J(1, a \sqrt{q}) q^{2} J(0, a \sqrt{q}) \ln (q / L)-4 J a q^{3 / 2} J(0, a \sqrt{q})^{2} \ln (q / L) \\
& +6 q^{2} J(1, a \sqrt{q})-6 q^{2} J(0, a \sqrt{q}) J(1, a \sqrt{q})-12 J q J(1, a \sqrt{q}) \\
& +8 J J(1, a \sqrt{q}) q J(0, a \sqrt{q}) \ln (q / L)-8 J J(1, a \sqrt{q}) q \ln (q / L) \\
& +12 J q J(0, a \sqrt{q}) J(1, a \sqrt{q})), \\
n_{g}^{(2113) G=}= & a q\left(a q^{3 / 2} J(0, a \sqrt{q})^{2} \ln (q / L)^{2}+2 q J(1, a \sqrt{q}) \ln (q / L)^{2}\right. \\
& -2 J(1, a \sqrt{q}) q J(0, a \sqrt{q}) \ln (q / L)^{2}-a q^{3 / 2} J(0, a \sqrt{q}) \ln (q / L)^{2} \\
& -4 q J(0, a \sqrt{q}) \ln (q / L) J(1, a \sqrt{q})+4 q \ln (q / L) J(1, a \sqrt{q}) \\
& +8 J J(0, a \sqrt{q}) \ln (q / L) J(1, a \sqrt{q})-8 J \ln (q / L) J(1, a \sqrt{q}) \\
& -4 J J(1, a \sqrt{q}) \ln (q / L)^{2}+4 J J(1, a \sqrt{q}) J(0, a \sqrt{q}) \ln (q / L)^{2} \\
& \left.+2 J a q^{1 / 2} J(0, a \sqrt{q}) \ln (q / L)^{2}-2 J a q^{1 / 2} J(0, a \sqrt{q})^{2} \ln (q / L)^{2}\right) .
\end{aligned}
$$

The 22-factors can be cascaded into the following form

$$
\begin{aligned}
& n_{g}^{(220) G}=n_{g}^{(2202) G} p^{2}+n_{g}^{(2203) G} p^{3}+n_{g}^{(2204) G} p^{4} \\
& n_{g}^{(221) G}=n_{g}^{(2212) G} p^{2}+n_{g}^{(2213) G} p^{3}+n_{g}^{(2214) G} p^{4}
\end{aligned}
$$

with the individual sub-factors

$$
\begin{aligned}
n_{g}^{(2202) G}= & 2 \sqrt{J} \sqrt{J-q}\left(4 J(1, a \sqrt{q}) a q J(0, a \sqrt{q})-4 a^{2} q^{3 / 2} J(0, a \sqrt{q})^{2}\right. \\
& -4 J(1, a \sqrt{q}) a q+4 a^{2} q^{3 / 2} J(0, a \sqrt{q})-5 q^{3 / 2} J(1, a \sqrt{q})^{2} a^{2} \\
& \left.+12 \sqrt{q}+12 \sqrt{q} J(0, a \sqrt{q})^{2}-24 \sqrt{q} J(0, a \sqrt{q})\right) \\
n_{g}^{(2203) G}= & 4 \sqrt{J} \sqrt{J-q}\left(-q^{3 / 2} J(1, a \sqrt{q})^{2} a^{2} \ln (q / L)+2 a^{2} q^{3 / 2} J(0, a \sqrt{q}) \ln (q / L)\right. \\
& -2 a^{2} q^{3 / 2} J(0, a \sqrt{q})^{2} \ln (q / L)+8 J(1, a \sqrt{q}) a q J(0, a \sqrt{q}) \ln (q / L) \\
& -8 J(1, a \sqrt{q}) a q \ln (q / L)+10 J(1, a \sqrt{q}) a q J(0, a \sqrt{q}) \\
& -10 q J(1, a \sqrt{q}) a+12 \sqrt{q} J(0, a \sqrt{q})^{2} \ln (q / L)+12 \sqrt{q} \ln (q / L) \\
& \left.+16 \sqrt{q}+16 \sqrt{q} J(0, a \sqrt{q})^{2}-24 \sqrt{q} J(0, a \sqrt{q}) \ln (q / L)-32 \sqrt{q} J(0, a \sqrt{q})\right), \\
n_{g}^{(2204) G}= & 2 \sqrt{J} \sqrt{J-q}\left(q^{3 / 2} J(1, a \sqrt{q})^{2} a^{2} \ln (q / L)^{2}+4 q J(0, a \sqrt{q}) \ln (q / L) J(1, a \sqrt{q}) a\right. \\
& +4 \sqrt{q}-4 J(1, a \sqrt{q}) a q \ln (q / L)^{2}+4 J(1, a \sqrt{q}) a q J(0, a \sqrt{q}) \ln (q / L)^{2} \\
& -4 q \ln (q / L) J(1, a \sqrt{q}) a+4 \sqrt{q} J(0, a \sqrt{q})^{2} \ln (q / L)^{2}+4 \sqrt{q} \ln (q / L)^{2} \\
& +4 \sqrt{J-q} J(0, a \sqrt{q})^{2}-8 \sqrt{q} J(0, a \sqrt{q})-8 \sqrt{q} J(0, a \sqrt{q}) \ln (q / L)^{2} \\
& \left.+8 \sqrt{q} \ln (q / L)+8 \sqrt{q} J(0, a \sqrt{q})^{2} \ln (q / L)-16 \sqrt{q} J(0, a \sqrt{q}) \ln (q / L)\right) .
\end{aligned}
$$

The 221-factors are given by the following expressions

$$
\begin{aligned}
n_{g}^{(2212) G}= & a\left(3 a q^{5 / 2} J(0, a \sqrt{q})^{2}-3 a q^{5 / 2} J(0, a \sqrt{q})+4 q^{5 / 2} J(1, a \sqrt{q})^{2} a\right. \\
& +6 J a q^{3 / 2} J(0, a \sqrt{q})-6 J(1, a \sqrt{q}) q^{2} J(0, a \sqrt{q})+6 J(1, a \sqrt{q}) q^{2} \\
& -6 J a q^{3 / 2} J(0, a \sqrt{q})^{2}-8 J q^{3 / 2} J(1, a \sqrt{q})^{2} a-12 J q J(1, a \sqrt{q}) \\
& +12 J J(1, a \sqrt{q}) q J(0, a \sqrt{q})), \\
n_{g}^{(2213) G}= & 2 a q\left(2 q^{3 / 2} J(1, a \sqrt{q})^{2} a \ln (q / L)+3 a q^{3 / 2} J(0, a \sqrt{q})^{2} \ln (q / L)\right. \\
& -3 a q^{3 / 2} J(0, a \sqrt{q}) \ln (q / L)-4 J q^{1 / 2} J(1, a \sqrt{q})^{2} a \ln (q / L) \\
& +6 J(1, a \sqrt{q}) q \ln (q / L)-6 J(1, a \sqrt{q}) q J(0, a \sqrt{q}) \ln (q / L) \\
& +6 J a q^{1 / 2} J(0, a \sqrt{q}) \ln (q / L)-6 J a q^{1 / 2} J(0, a \sqrt{q})^{2} \ln (q / L) \\
& -10 q J(0, a \sqrt{q}) J(1, a \sqrt{q})+12 J J(1, a \sqrt{q}) J(0, a \sqrt{q}) \ln (q / L)
\end{aligned}
$$




$$
\begin{aligned}
& +10 q J(1, a \sqrt{q})-12 J J(1, a \sqrt{q}) \ln (q / L)-20 J J(1, a \sqrt{q}) \\
& +20 J J(0, a \sqrt{q}) J(1, a \sqrt{q})), \\
n_{g}^{(2214) G}= & a q\left(a q^{3 / 2} J(0, a \sqrt{q})^{2} \ln (q / L)^{2}-a q^{3 / 2} J(0, a \sqrt{q}) \ln (q / L)^{2}\right. \\
& -2 J(1, a \sqrt{q}) q J(0, a \sqrt{q}) \ln (q / L)^{2}+2 J a q^{1 / 2} J(0, a \sqrt{q}) \ln (q / L)^{2} \\
& -2 J a q^{1 / 2} J(0, a \sqrt{q})^{2} \ln (q / L)^{2}+2 q J(1, a \sqrt{q}) \ln (q / L)^{2} \\
& +4 q \ln (q / L) J(1, a \sqrt{q})-4 q J(0, a \sqrt{q}) \ln (q / L) J(1, a \sqrt{q}) \\
& -4 J J(1, a \sqrt{q}) \ln (q / L)^{2}+4 J J(1, a \sqrt{q}) J(0, a \sqrt{q}) \ln (q / L)^{2} \\
& +8 J J(0, a \sqrt{q}) \ln (q / L) J(1, a \sqrt{q})-8 J \ln (q / L) J(1, a \sqrt{q})) .
\end{aligned}
$$

Similarly, the 23 -factors turn out to be

$$
\begin{aligned}
& n_{g}^{(230) G}=n_{g}^{(2303) G} p^{3}+n_{g}^{(2304) G} p^{4}, \\
& n_{g}^{(231) G}=n_{g}^{(2313) G} p^{3}+n_{g}^{(2314) G} p^{4} . \\
& n_{g}^{(2303) G}=2 \sqrt{J} \sqrt{q} \sqrt{J-q}\left(2 a^{2} q J(0, a \sqrt{q})-2 a^{2} q J(0, a \sqrt{q})^{2}-3 q J(1, a \sqrt{q})^{2} a^{2}\right. \\
& \left.+4 J(0, a \sqrt{q})^{2}+4-8 J(0, a \sqrt{q})\right), \\
& n_{g}^{(2304) G}=4 \sqrt{J} \sqrt{J-q}\left(a^{2} q^{3 / 2} J(0, a \sqrt{q}) \ln (q / L)-a^{2} q^{3 / 2} J(0, a \sqrt{q})^{2} \ln (q / L)\right. \\
& -q^{3 / 2} J(1, a \sqrt{q})^{2} a^{2} \ln (q / L)+2 a q J(1, a \sqrt{q}) J(0, a \sqrt{q}) \ln (q / L) \\
& -2 a q J(1, a \sqrt{q}) \ln (q / L)+4 \sqrt{q} \ln (q / L)+4 J(1, a \sqrt{q}) a q J(0, a \sqrt{q}) \\
& +4 \sqrt{q} J(0, a \sqrt{q})^{2} \ln (q / L)-4 J(1, a \sqrt{q}) a q+6 \sqrt{q}+6 \sqrt{q} J(0, a \sqrt{q})^{2} \\
& -8 \sqrt{q} J(0, a \sqrt{q}) \ln (q / L)-12 \sqrt{q} J(0, a \sqrt{q})),
\end{aligned}
$$

and

$$
\begin{aligned}
n_{g}^{(2313) G}= & a q\left(a q^{3 / 2} J(0, a \sqrt{q})^{2}-a q^{3 / 2} J(0, a \sqrt{q})+2 q^{3 / 2} J(1, a \sqrt{q})^{2} a\right. \\
& +2 q J(1, a \sqrt{q})-2 J(1, a \sqrt{q}) q J(0, a \sqrt{q})+2 J a q^{1 / 2} J(0, a \sqrt{q}) \\
& -2 J a q^{1 / 2} J(0, a \sqrt{q})^{2}-4 J q^{1 / 2} J(1, a \sqrt{q})^{2} a-4 J J(1, a \sqrt{q}) \\
& +4 J J(1, a \sqrt{q}) J(0, a \sqrt{q})) \\
n_{g}^{(2314) G}= & 2 a q\left(a q^{3 / 2} J(0, a \sqrt{q})^{2} \ln (q / L)+q^{3 / 2} J(1, a \sqrt{q})^{2} a \ln (q / L)\right. \\
& -a q^{3 / 2} J(0, a \sqrt{q}) \ln (q / L)-2 J q^{1 / 2} J(1, a \sqrt{q})^{2} a \ln (q / L) \\
& -2 J a q^{1 / 2} J(0, a \sqrt{q})^{2} \ln (q / L)-2 J(1, a \sqrt{q}) q J(0, a \sqrt{q}) \ln (q / L) \\
& +2 q J(1, a \sqrt{q}) \ln (q / L)+2 J a q^{1 / 2} J(0, a \sqrt{q}) \ln (q / L)+4 q J(1, a \sqrt{q}) \\
& -4 q J(0, a \sqrt{q}) J(1, a \sqrt{q})+4 J J(1, a \sqrt{q}) J(0, a \sqrt{q}) \ln (q / L) \\
& -4 J J(1, a \sqrt{q}) \ln (q / L)+8 J J(0, a \sqrt{q}) J(1, a \sqrt{q})-8 J J(1, a \sqrt{q})) .
\end{aligned}
$$

Finally, the $l(p)^{3}$ terms are given by

$$
n_{g}^{(3) G}=n_{g}^{(30) G}+(q / L)^{p} n_{g}^{(31) G}+\left((q / L)^{p}\right)^{2} n_{g}^{(32) G}+\left((q / L)^{p}\right)^{3} n_{g}^{(33) G},
$$

where the factors are expressed as

$$
\begin{aligned}
n_{g}^{(30) G} & =n_{g}^{(300) G}+f n_{g}^{(301) G} \\
n_{g}^{(31) G} & =n_{g}^{(310) G}+f n_{g}^{(311) G}, \\
n_{g}^{(32) G} & =n_{g}^{(320) G}+f n_{g}^{(321) G}, \\
n_{g}^{(33) G} & =n_{g}^{(330) G}+f n_{g}^{(331) G} .
\end{aligned}
$$




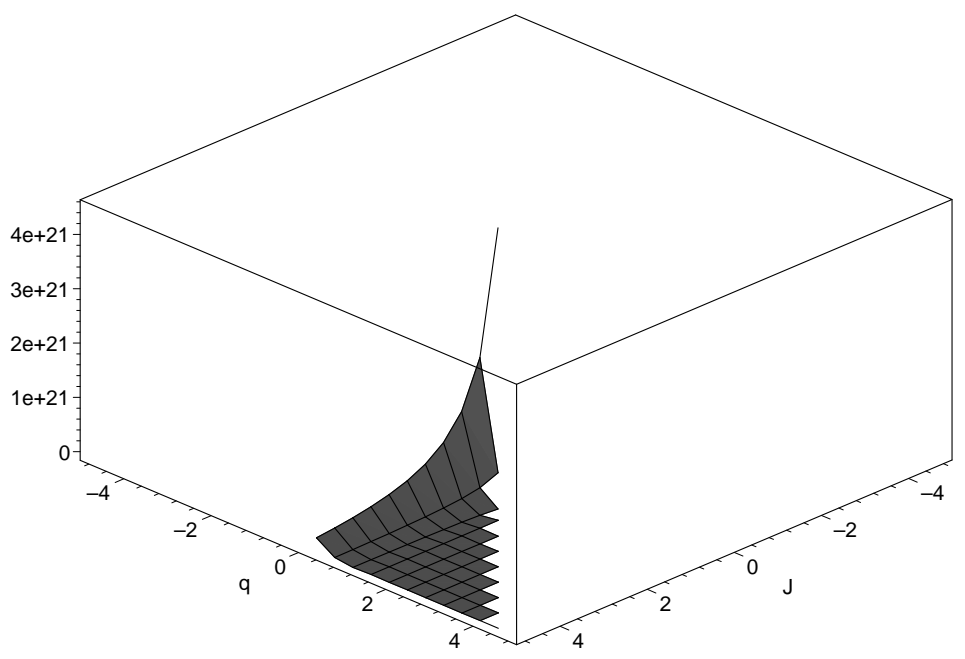

Figure 17: The dimensional behavior of the determinant of the metric tensor $\|g\|$ plotted as a function of the scale and angular momentum, viz., $q, J$, describing the nature of the stability of the general massive rotating quarkonia.

The 30-factors are given by the following expressions

$$
\begin{aligned}
n_{g}^{(300) G}= & 2 a q \sqrt{J} \sqrt{J-q}\left(a q^{1 / 2} J(0, a \sqrt{q})^{2}-a q^{1 / 2} J(0, a \sqrt{q})\right. \\
& \left.+2 J(1, a \sqrt{q}) J(0, a \sqrt{q})+2 q^{1 / 2} J(1, a \sqrt{q})^{2} a-2 J(1, a \sqrt{q})\right), \\
n_{g}^{(301) G=} & a^{2} q^{3 / 2} J(1, a \sqrt{q})^{2}(2 J-q) .
\end{aligned}
$$

The 31-factors take the following forms

$$
\begin{aligned}
& n_{g}^{(310) G}=6 a p q \sqrt{J} \sqrt{J-q}\left(a q^{1 / 2} J(0, a \sqrt{q})^{2}-a q^{1 / 2} J(0, a \sqrt{q})\right. \\
& \left.-2 J(1, a \sqrt{q})+2 J(0, a \sqrt{q}) J(1, a \sqrt{q})+2 q^{1 / 2} J(1, a \sqrt{q})^{2} a\right), \\
& n_{g}^{(311) G}=3 a^{2} q^{3 / 2} J(1, a \sqrt{q})^{2} p(2 J-q) .
\end{aligned}
$$

Similarly, the 32 -factors turn out to be

$$
\begin{aligned}
& n_{g}^{(320) G}=6 a q p^{2} \sqrt{J} \sqrt{J-q}\left(a q^{1 / 2} J(0, a \sqrt{q})^{2}-a q^{1 / 2} J(0, a \sqrt{q})\right. \\
& \left.-2 J(1, a \sqrt{q})+2 q^{1 / 2} J(1, a \sqrt{q})^{2} a+2 J(0, a \sqrt{q}) J(1, a \sqrt{q})\right), \\
& n_{g}^{(321) G}=3 a^{2} p^{2} q^{3 / 2} J(1, a \sqrt{q})^{2}(2 J-q) .
\end{aligned}
$$

Finally, the 33 -factors can be obtained as follows

$$
\begin{aligned}
n_{g}^{(330) G}= & 2 a q p^{3} \sqrt{J} \sqrt{J-q}\left(a q^{1 / 2} J(0, a \sqrt{q})^{2}-a q^{1 / 2} J(0, a \sqrt{q})\right. \\
& \left.+2 J(1, a \sqrt{q}) J(0, a \sqrt{q})-2 J(1, a \sqrt{q})+2 q^{1 / 2} J(1, a \sqrt{q})^{2} a\right), \\
n_{g}^{(331) G}= & a^{2} p^{3} J(1, a \sqrt{q})^{2} J(1, a \sqrt{q})^{2}(2 J-q) .
\end{aligned}
$$

From the three parameter quarkonia, the Fig.(17) offers the general three dimensional behavior of the determinant of the metric tensor. From the viewpoint of the fixed $p$ quarkonia in the Regge regime as previously 
described, we notice in the present case that the graph of the determinant of the metric tensor shows the similar nature of the Gaussian fluctuations of the strong QCD coupling. Notice that the underlying quarkonium configuration may become unstable in specific regions of the parameters. For instance, for small values of the momentum scale and angular momentum, viz $q, J \rightarrow 0^{+}$, we observe that the determinant of the metric tensor picks up a large amplitude of order $10^{21}$. However, for intermediate values of $q$ and angular momentum $q, J$, the Fig. (17) shows that the underlying configuration is well-stable and so is the basis for the three dimension intrinsic manifold $\left(M_{3}, g\right)$. From the observation of the Fig. (17), we predict that the regions of the thermodynamic stability are present in the interval $q, J \in(1,4)$. Globally, the stability of $\left(M_{3}, g\right)$ constraint the principle minors $\left\{g_{i i}, p_{S}^{G},\|g\|\right\}$ to remain positive. Specifically, for the same sign of $\{b, p, f\}$, the volume stability of the $\left(M_{3}, g\right)$ imposes the following constraint

$$
\begin{aligned}
n_{g}^{(0) G}+n_{g}^{(1) G} l(p)+n_{g}^{(2) G} l(p)^{2}+n_{g}^{(3) G} l(p)^{3} & >0, J(0, a \sqrt{q})<1 \\
& <0, J(0, a \sqrt{q})<1
\end{aligned}
$$

Importantly, it is worth mentioning that both the limiting configurations with $J=q$ and $J(0, a \sqrt{q})=1$ are abided from the thermodynamic stability constraints. To summarize the phases of generic quarkonia, the exact formula for the scalar curvature may analogously be deduced as the one we have offered for the $q J$-plane. With some modification of the pre-factors, we find that the summation over $l(p)$ naturally arises with the $B_{n}$ as the polynomials in $p$, whose coefficients can be expressed as the functions of the Bloch-Nordsieck logarithmic factor $f(q, J)$. In the present case, the denominator of the scalar curvature involves the third and second power of the factors in the numerator of the determinant of the metric tensor. Interestingly, the quantitative property of the scalar curvature follows, except the fact that its explicit presentation is a bit prolonged. Up to a phase of QCD, we observe that the global properties of the three parameter quarkonia remain the same as we have exactly indicated for the $Q J$ - and $Q M$-planes.

\section{Discussion and Conclusion}

We have studied the thermodynamic geometric properties of non-abelian quarkonium bound states arising from the consideration of the one-loop gluon confining QCD potentials. Following the Polyakov argument, the thermodynamic intrinsic geometric nature of the QCD coupling is analyzed for the case of the Coulambic and rising confining regimes. Without any approximation, the effect of Regge and Bloch-Nordsieck rotations is explored form the stability perspective of the strongly coupled quarkonia with and without the inclusion of the effects coming from the mass. The fact that once the quark matter being formed should be stable, motivated us to determine the values of the parameters of strongly coupled quarkonia. We have obtained the allowed range of parameters from the stability conditions of the thermodynamic geometry. We have initiated our analysis with the consideration of massless non-rotating quarkonia. In this case, we have derived that the thermodynamic stability constraints impose the requirements $g_{i i}>0$ and $\|g\|>0$. Given the momentum scale $q$ and index $p$ of the configuration, our analysis determines an intrinsically stable inter dependence of the parameter from one and the other. The Regge rotating quarkonia is geometrically trivial because it only requires the positive sign of the rotation term. With and without the Regge rotation, the domain of the index $p(q)$ can be offered as

$$
\mathcal{P}_{m=0}:=\left\{p \mid g_{i i}>0,\|g\|>0\right\}
$$

In the case of massive rotating quarkonia, there is a set of thermodynamic stability constraints on the parameters which arises from the fluctuations of the q-angular momentum plane, q-mass plane and the entire q-angular momentum-index manifold, when all the parameters of the configuration are allowed to fluctuate. It turns out that one can fix all the parameters of the configuration following from one constraint to the other. One such procedure is to respectively consider the fluctuations on the $Q J$-plane, then on the $Q M$-plane and finally on the $Q p J$-manifold. In the case of the $Q J$-plane, it follows that we determine the thermodynamically stable $Q^{2}(J)$ directly from the fluctuations of the Bloch-Nordsieck rotation. By taking an account of the thermodynamic stability constraint of the $Q^{2}(J)$, we can subsequently determine the thermodynamically stable domain of the mass $M(Q)$ on the $Q M$-plane. Finally, the thermodynamically stable domain of the index $p$ can be determined as the function of $Q$ and $J$ on the $Q p J$-manifold. Thus, the consideration of the thermodynamic fluctuation theory offers the determination of the stable domains of the QCD strong coupling. This follows from the fact that the stable domain of $q$ and the Bloch-Nordsieck rotation can be determined from one another, and thus the index can solely be expressed as a function of one the either two. This shows the power of the thermodynamic stability condition that determines all possible stable phases of the strongly coupled quarkonia. 
Based on the analysis of the present paper, the thermodynamically stable index of the Bloch-Nordsieck rotating massive quarkonia is constrained by the following set

$$
\mathcal{P}_{m \neq 0}:=\left\{p \mid n_{i i}^{G}>0, n_{S}^{G}>0, n_{g}^{G}>0\right\} .
$$

To be specific, we have introduced the intrinsic geometric notion to QCD thermodynamics, and thereby it has been applied to study the behavior of constant and variable index strong QCD coupling in several simple thermodynamic considerations. Our explicit computations of the local and global thermodynamic corrections provide a prominent realization of the limiting equilibrium thermodynamic configuration of the strongly coupled QCD. This offers an intrinsic geometric exercise of the quarkonia, which has been described by the parameters of the strong coupling potential. The scalar curvature of the underlying thermodynamic metric tensor takes an exact form, which signifies the correlation volume of the underlying quarkonia. With and without the variable index strong QCD coupling, our consideration of the underlying thermodynamic fluctuations indicates interesting relationships between the geometrical concepts of quarkonium thermodynamics and phases of the massless, Regge rotating and Bloch-Nordsieck rotating quarkonia. For the case of the Bloch-Nordsieck rotating quarkonia, we have implemented the above constraints one by one, and thereby we conclude that the thermodynamically stable domain of the parameters is highly constrained on the $\left(M_{3}, g\right)$. For the value of $p=5 / 6$ which generically corresponds to the Regge rotating quarkonia, we observe that all the stability constraints are satisfied, whenever $q, J \in(1,4)$. Thermodynamically, this determines all possible intermediate stable phases of the strongly coupled quarkonia. As the gluonic interactions become softer and softer, we find in the limit of Bloch-Nordsieck resummation that the underlying Sudhakov form factor offers the thermodynamic stability properties of the $\pi, K$ and $D_{s}$ particles.

Mathematically, we have computed the intrinsic geometric properties of the Bessel function of first kind convoluted with two logarithmic functions of the respective weights $(0,2 p)$. Explicitly, we determine the domain of the convolution of the zeroth order Bessel function $J(0, a \sqrt{q})$, where the configuration finds globally stable fixed points and remains globally regular on the intrinsic three manifold $\left(M_{3}, g\right)$. Notice that the exact determination of the global stability set $\mathcal{P}_{m \neq 0}$ is consistent with the local stability constraints on $\left(M_{3}, g\right)$. Geometrically, we find that the global stability requirement of $\left(M_{3}, g\right)$ avoids the limit $J(0, a \sqrt{q}) \rightarrow 1$. From the perspective of the intrinsic Riemannian geometry, it would be worth to find the globally stable and globally regular domains of the convoluted Bessel functions with finitely many elementary functions of distinct weights. This study herewith anticipates further examination of the globally stable and regular mapping class convolutions from the perspective of intrinsic Riemannian geometry.

Physically, it would be interesting to analyze whether this kind of approach can be pushed further to explore intrinsic geometric properties of strong coupling QCD thermodynamic correlations. Specifically, we would like to understand the modifications towards the elements of the set $\mathcal{P}_{m \neq 0}$ and the associated Ricci scalar curvature, which we have explicitly offered for the $Q J$-plane. This geometric initiation would take a better shape once we take finite size effects of higher order perturbative QCD into account, and would reach its final goal with the complete account of non-perturbative QCD. The geometric feature of these explorations is left for the future.

\section{Acknowledgment}

The work of S. B. has been supported in part by the European Research Council grant n. 226455, "SUPERSYMMETRY, QUANTUM GRAVITY AND GAUGE FIELDS (SUPERFIELDS)". BNT thanks Prof. V. Ravishankar for support and encouragement. The work of BNT has been supported by a postdoctoral research fellowship of the "INFN-Laboratori Nazionali di Frascati, Roma, Italy".

\section{References}

[1] V. V. Khoze, A. Ringwald, Phys. Lett B 259106 (1991).

[2] T. Matsui and H. Satz, Phys. Lett. B 178 (1986) 416;

[3] F. Karsch, M.-T. Mehr and H. Satz, Z. Physik C 37 (1988) 617.

[4] N. Brambilla et. al, arXiv:hep-ph/0412158 and Refs, therein.

[5] Vinod Chandra, A. Ranjan, V. Ravishankar, Euro. Phys. J A bf 40, 109 (2009):arXiv:0801.1286.

[6] Vineet Agotiya, Vinod Chandra, B. K. Patra, Phys. Rev C 80, 025210 (2009) 
[7] Vinod Chandra, V. Ravishankar, arXiv:1006.3995.

[8] STAR colloboration, Nucl. Phys. A 737, 102 (2005).

[9] C. E. Aguiar, E. S. Fraga, T. Kodama, Braz. J. Phys. 34 no.1a Sao Paulo Mar. (2004).

[10] PHENIX Collaboration, Phys. Rev. Lett. 98, 232301 (2007); Phys. Rev. Lett. 98232002 (2007); arXiv: 0903.2041.

[11] A. Strominger, C. Vafa, Phys. Lett. B 37999 (1996), arXiv:hep-th/9601029v2.

[12] A. Sen, Mod. Phys. Lett. A 102081 (1995), arXiv:hep-th/9504147v2.

[13] A. Dabholkar, Phys. Rev. Lett. 94241301 (2005), arXiv:hep-th/0409148v2.

[14] L. Andrianopoli, R. D'Auria, S. Ferrara, arXiv:hep-th/9707203v1.

[15] A. Dabholkar, F. Denef, G. W. Moore, B. Pioline, JHEP 0510096 (2005), arXiv:hep-th/0507014 1.

[16] A. Dabholkar, F. Denef, G. W. Moore, B. Pioline, JHEP 0508021 (2005), arXiv:hep-th/0502157v4.

[17] A. Sen, JHEP 0507073 (2005), arXiv:hep-th/0505122v2.

[18] J. P. Gauntlett, J. B. Gutowski, C. M. Hull, S. Pakis, H. S. Reall, Class. Quant. Grav. 204587 (2003), arXiv:hep-th/0209114v3.

[19] J. B. Gutowski, H. S. Reall, JHEP 0404048 (2004), arXiv:hep-th/0401129v3.

[20] I. Bena, N. P. Warner, Adv. Theor. Math. Phys. 9667 (2005), arXiv:hep-th/0408106v2.

[21] J. P. Gauntlett, J. B. Gutowski, Phys. Rev. D 71045002 (2005), arXiv:hep-th/0408122v3.

[22] T. Sarkar, G. Sengupta, B. N. Tiwari, JHEP 0611 (2006) 015, arXiv:hep-th/0606084v1.

[23] T. Sarkar, G. Sengupta, B. N. Tiwari, JHEP 0810, 076, (2008), arXiv:0806.3513v1 [hep-th].

[24] B. N. Tiwari, Proc. Quantum Gravity, Hoelback 2008, arXiv:0801.4087v1 [hep-th].

[25] S. Bellucci, B. N. Tiwari, arXiv:0808.3921v1 [hep-th].

[26] S. Bellucci, B. N. Tiwari, JHEP 05023 (2010), arXiv:0910.5314v2 [hep-th].

[27] G. Ruppeiner, Rev. Mod. Phys 67605 (1995), Erratum 68 (1996) 313.

[28] G. Ruppeiner, Phys. Rev. A 201608 (1979).

[29] G. Ruppeiner, Phys. Rev. Lett. 50287 (1983).

[30] G. Ruppeiner, Phys. Rev. A 271116 (1983).

[31] G. Ruppeiner and C. Davis, Phys. Rev. A 412200 (1990).

[32] G. Ruppeiner, Phy. Rev. D 78, 024016 (2008).

[33] B. N. Tiwari, Vinod Chandra, Subhashish Banerjee, arXiv:1008.2853,

[34] Behrouz Mirza, Hosein Mohammadzadeh, Phys. Rev. E 80, 011132 (2009).

[35] J. E. Aman, I. Bengtsson, N. Pidokrajt, Gen. Rel. Grav. 381305 (2006), arXiv:gr-qc/0601119v1.

[36] J. Shen, R. G. Cai, B. Wang, R. K. Su, Int. J. Mod. Phys. A 2211 (2007), arXiv:gr-qc/0512035v1.

[37] J. E. Aman, I. Bengtsson, N. Pidokrajt, Gen. Rel. Grav. 351733 (2003), arXiv:gr-qc/0304015v1.

[38] J. E. Aman, N. Pidokrajt, Phys. Rev. D 73024017 (2006), arXiv:hep-th/0510139v3.

[39] S. Ferrara, R. Kallosh, A. Strominger, Phys. Rev. D 52 R5412 (1995), arXiv:hep-th/9508072v3.

[40] Andrew Strominger, Phys. Lett. B 38339 (1996), arXiv:hep-th/9602111v3.

[41] S. Ferrara, R. Kallosh, Phys. Rev. D 54 (1996) 1514-1524, arXiv:hep-th/9602136.

[42] S. Ferrara, G. W. Gibbons, R. Kallosh, Nucl. Phys. B 50075 (1997), arXiv:hep-th/9702103.

[43] S. Bellucci, S. Ferrara, A. Marrani, Fortsch. Phys. 56761 (2008), arXiv:0805.1310,

[44] F. Weinhold, J. Chem. Phys. 63 , 2479 (1975), DOI:10.1063/1.431689.

[45] F. Weinhold, J. Chem. Phys 63, 2484 ( 1975).

[46] S. Bellucci, V. Chandra, B. N. Tiwari, arXiv:0812.3792v1 [hep-th].

[47] V. Baluni, Phys. Rev. D 172092 (1978).

[48] R. Jakob, P. Kroll, Phys. Lett. B 315 (1993) 463; B 319 (1993) 545(E). 
[49] H-n. Li, G. Sterman, Nucl. Phys. B 381, 129 (1992).

[50] A. M. Polyakov, JETP Lett. 20194 (1974).

[51] I. E. Dzyaloshinskii, JEPT. Lett. Vol. 37, No. 4, 20 February 1983, ® American Institute of Physics 0021-3640/ 83/04227-0201.00.

[52] J. M. Campbell, R. K. Ellis, Phys. Rev. D 60, 113006 (1999).

[53] A. Grau, G. Pancheri, and Y. N. Srivastava, Phys. Rev. D 60, 114020 (1999).

[54] L.N. Liptov and V. S. Fadin, JEPT. Lett. Vol. 49, No. 6, 25 March 1989, ® American Institute of Physics 0021-3640/ 89/060352-0501.00.

[55] V. Sudakov, Zh. Eksp. Teor. Fiz. 3087 (1956); (Eng. trans) Sov. Phys. JETP 365 (1956).

[56] F. Bloch and A. Nordsieck, Phys. Reports 5254 (1937).

[57] R.D. Tangerman, P.J. Mulders, Phys. Rev. D 513357 (1995).

[58] G. Pancheri, A. Grau, R. M. Godbole, Y. N. Srivastava, arXiv:1007.0208v1 [hep-ph]

[59] H. Pagels, S. Stokar, Phys. Rev. D 20, 2947 (1979)

[60] J. C. Collins, Adv. Ser. Direct. High Energy Phys. 5573 (1989).

[61] L. Durand, Phys. Rev. D 321257 (1985).

[62] J. H. Kuhn, XXIV Cracow School of Theoretical Physics, Zakopane, Poland, CERN-TH/ 4083 (1984). 